Supporting Information

\title{
Cobalt-Catalyzed Regiodivergent Stereoselective Hydroboration of 1,3-Diynes to Access Boryl- Functionalized Enynes
}

Hui Leng Sang, Caizhi Wu, Guan Ge Darren Phua and Shaozhong Ge*

Department of Chemistry, National University of Singapore, 3 Science Drive 3, Singapore 117543, Singapore

Email: chmgsh@nus.edu.sg

Table of Contents

General Remarks

General procedure for Co-catalyzed $\beta$-hydroboration of 1,3-diynes \& Characterization

Data of Isolated Products

General procedure for Co-catalyzed $\alpha$-hydroboration of 1,3-diynes \& Characterization Data of Isolated Products

Determination of regio- and stereochemistry of 2e' and 3e' by NOE and 2D HMBC analysis

General procedure for gram scale for Co-catalyzed $\beta$-hydroboration of 1,3-diynes

General procedure for gram scale for Co-catalyzed $\alpha$-hydroboration of 1,3-diynes

Deuterium studies

NMR Spectra $\left({ }^{1} \mathrm{H},{ }^{13} \mathrm{C}\{\mathrm{H}\}\right.$ and $\left.{ }^{19} \mathrm{~F}\right)$ 


\section{General Remarks}

All the manipulations were performed in an argon-filled glovebox, unless mentioned otherwise. THF, toluene, and hexane were purified by passing the degassed solvents $\left(\mathrm{N}_{2}\right)$ through a column of activated alumina (solvent purification system purchased from Innovative Technologies, Newburyport, MA). The following chemicals were purchased and used as received: Co(acac) 2 (99\%, Sigma-Aldrich), HBpin (Oakwood Chemicals). Symmetrical 1,3-diynes were prepared by Copper-catalyzed homocoupling of the respective alkynes. ${ }^{1}$ Unsymmetrical 1,3diynes were prepared by Glaser coupling of the corresponding alkynes with ethynyltrimethylsilane according to previously reported procedures. ${ }^{2}$ All other reagents and solvents were purchased from commercial sources and used without purification.

${ }^{1} \mathrm{H}$ and ${ }^{13} \mathrm{C}$ spectra were recorded using Bruker $300 \mathrm{MHz}, 400 \mathrm{MHz}$, or $500 \mathrm{MHz}$ NMR spectrometers. ${ }^{1} \mathrm{H}$ NMR and ${ }^{13} \mathrm{C}$ NMR spectra were referenced to resonances of the residual signals of the deuterated solvents. As such, the ${ }^{1} \mathrm{H}$ and ${ }^{13} \mathrm{C}$ signals of $\mathrm{CDCl}_{3}$ were calibrated to $7.26 \mathrm{ppm}$ (singlet) and $77.16 \mathrm{ppm}$ (triplet) respectively. Multiplicities are recorded as: $\mathrm{s}=$ singlet, $\mathrm{d}=$ doublet, $\mathrm{t}=$ triplet, $\mathrm{dd}=$ doublet of doublets, $\mathrm{dt}=$ doublet of triplets and $\mathrm{m}=$ multiplet. $\mathrm{GC}$ analysis was acquired on Agilent 6850 gas chromatograph equipped with a flameionization detector. HR-MS analyses were performed using Agilent GC-QTOF. GC-MS analysis was performed on Shimadzu GC-2010 gas chromatograph coupled to a Shimadzu QP2010 mass selective detector. 


\section{General procedure for Co-catalyzed $\beta$-hydroboration of 1,3-diynes}

In an Ar-filled glovebox, a mixture of $\mathrm{Co}(\mathrm{acac})_{2}(2.4 \mathrm{mg}, 10.0 \mu \mathrm{mol})$ and xantphos $(5.8 \mathrm{mg}, 10.0 \mu \mathrm{mol})$ in THF $(0.2 \mathrm{~mL})$ was added into a $4-\mathrm{mL}$ screw-capped vial containing a magnetic stirring bar. The resulting mixture was stirred for 2 minutes before adding HBpin $(36.0 \mathrm{mg}, 0.280 \mathrm{mmol})$ and 1,3-diynes $(0.250 \mathrm{mmol})$ successively. The reaction mixture was stirred at $40^{\circ} \mathrm{C}$ for 24 hours.

Note: As the Bpin derivatives are stable in column chromatography to a limited extent, most of the boronsubstituted enynes were further converted to its Bdan derivatives to facilitate the isolation process.

Upon reaction completion, $\mathrm{FeCl}_{3}(3.2 \mathrm{mg}, 20.0 \mu \mathrm{mol}), 1,8$-diaminonaphthalene ( $40.0 \mathrm{mg}, 0.250 \mathrm{mmol}$ ), imidazole (34.0 $\mathrm{mg}, 0.500 \mathrm{mmol}$ ) was added into another 4-mL screw-capped vial containing a magnetic stirring bar containing water $(0.5 \mathrm{~mL})$ and acetonitrile $(0.5 \mathrm{~mL})$. Subsequently, the crude hydroboration reaction mixture was transferred and rinsed with acetonitrile $(1.5 \mathrm{~mL})$ into the latter vial. It was then stirred for 12 to $18 \mathrm{hr}$ at room temperature for the conversion to Bdan derivatives to take place. ${ }^{3}$ After the completion of the reaction, the crude mixture was washed with saturated $\mathrm{NaCl}$ and extracted thrice with ethyl acetate. The organic layer was then dried over sodium sulfate, filtered and concentrated under vacuum. Lastly, the residue was purified by flash chromatography on silica using a mixture of ethyl acetate and hexane as eluent. The details for separation and characterization data of the products are stated below.

(Z)-2-(1-phenyl-4-(trimethylsilyl)but-1-en-3-yn-2-yl)-2,3-dihydro-1H-naphtho[1,8-de][1,3,2]diazaborinine (2a)<smiles>CC#CC(=Cc1ccccc1)C(C)(C)C</smiles>

The titled compound was isolated $(65.0 \mathrm{mg}, 71 \%, 98: 2)$ as yellow oil after chromatography on silica gel (100:1 hexane/EtOAc). ${ }^{1} \mathrm{H}$ NMR $\left(500 \mathrm{MHz}, \mathrm{CDCl}_{3}\right) \delta 8.08(\mathrm{~d}, J=8.4 \mathrm{~Hz}, 2 \mathrm{H}), 7.45$ $-7.37(\mathrm{~m}, 3 \mathrm{H}), 7.19-7.14(\mathrm{~m}, 2 \mathrm{H}), 7.11-7.06(\mathrm{~m}, 3 \mathrm{H}), 6.41(\mathrm{~d}, J=7.2 \mathrm{~Hz}, 2 \mathrm{H}), 6.08(\mathrm{~s}$, $2 \mathrm{H}), 0.35(\mathrm{~s}, 9 \mathrm{H}) .{ }^{13} \mathrm{C}\{\mathrm{H}\} \mathrm{NMR}\left(126 \mathrm{MHz}, \mathrm{CDCl}_{3}\right) \delta 144.9,141.0,136.8,136.5,129.6(2$ peaks overlap), 128.4, 127.7, 120.0, 118.0, 106.2, 105.6, 105.1, 0.1. The boron-bound carbon was not detected due to quadrupolar relaxation. HRMS (APCI) $m / z$ calcd for $\mathrm{C}_{23} \mathrm{H}_{23} \mathrm{BN}_{2} \mathrm{Si}$, $[\mathrm{M}-\mathrm{H}]^{+}$367.1802. Found: 367.1816.

\section{(Z)-2-(1-phenyl-4-(triethylsilyl)but-1-en-3-yn-2-yl)-2,3-dihydro-1H-naphtho[1,8-de][1,3,2]diazaborinine} (2b)

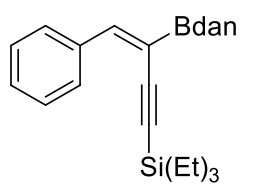

The titled compound was isolated $(79.2 \mathrm{mg}, 78 \%,>99:-)$ as yellow oil after chromatography on silica gel (100:1 hexane/EtOAc). ${ }^{1} \mathrm{H} \mathrm{NMR}\left(500 \mathrm{MHz}, \mathrm{CDCl}_{3}\right) \delta 8.14(\mathrm{~d}, J=6.9 \mathrm{~Hz}, 2 \mathrm{H})$, $7.45-7.38(\mathrm{~m}, 3 \mathrm{H}), 7.17(\mathrm{t}, J=7.7 \mathrm{~Hz}, 2 \mathrm{H}), 7.08(\mathrm{~d}, J=8.5 \mathrm{~Hz}, 3 \mathrm{H}), 6.40(\mathrm{~d}, J=7.2 \mathrm{~Hz}$, $2 \mathrm{H}), 6.13(\mathrm{~s}, 2 \mathrm{H}), 1.17(\mathrm{t}, J=7.9 \mathrm{~Hz}, 9 \mathrm{H}), 0.80(\mathrm{q}, J=7.9 \mathrm{~Hz}, 6 \mathrm{H}) .{ }^{13} \mathrm{C}\{\mathrm{H}\} \mathrm{NMR}(126 \mathrm{MHz}$, $\left.\mathrm{CDCl}_{3}\right) \delta 144.5,141.0,136.9,136.5,129.6,129.5,128.4,127.7,120.0,118.0,106.6,106.1$, 103.0, 7.8, 4.7. The boron-bound carbon was not detected due to quadrupolar relaxation. LRMS (EI) $\mathrm{m} / \mathrm{z}$ calcd for $\mathrm{C}_{26} \mathrm{H}_{29} \mathrm{BN}_{2} \mathrm{Si}$, 408.21. Found:408.00.

(Z)-2-(1-(2-methoxyphenyl)-4-(trimethylsilyl)but-1-en-3-yn-2-yl)-2,3-dihydro-1H-naphtho[1,8de][1,3,2] diazaborinine $(2 \mathrm{c})$<smiles>COc1ccccc1/C=C(\C#C[SiH3])CBr</smiles>

The titled compound was isolated $(79.7 \mathrm{mg}, 80 \%,>99:-)$ as yellow oil after chromatography on silica gel (50:1 hexane/EtOAc). ${ }^{1} \mathrm{H}$ NMR $\left(500 \mathrm{MHz}, \mathrm{CDCl}_{3}\right) \delta 7.85(\mathrm{~s}, 1 \mathrm{H}), 7.51(\mathrm{~d}, J=$ $7.6 \mathrm{~Hz}, 1 \mathrm{H}), 7.33(\mathrm{t}, J=7.9 \mathrm{~Hz}, 1 \mathrm{H}), 7.18-7.13(\mathrm{~m}, 2 \mathrm{H}), 7.08(\mathrm{~s}, 1 \mathrm{H}), 7.06(\mathrm{~d}, J=3.9 \mathrm{~Hz}$, $2 \mathrm{H}), 6.95(\mathrm{dd}, J=8.2,2.6 \mathrm{~Hz}, 1 \mathrm{H}), 6.40(\mathrm{~d}, J=7.2 \mathrm{~Hz}, 2 \mathrm{H}), 6.08(\mathrm{~s}, 2 \mathrm{H}), 3.87(\mathrm{~s}, 3 \mathrm{H}), 0.35$ $(\mathrm{s}, 9 \mathrm{H}) .{ }^{13} \mathrm{C}\{\mathrm{H}\} \mathrm{NMR}\left(126 \mathrm{MHz}, \mathrm{CDCl}_{3}\right) \delta 159.7,144.6,141.0,138.1,136.5,129.4,127.7$, $122.7,120.0,118.0,116.1,113.8,106.2$, 105.6 (2 peaks overlap), 55.4, 0.2. The boron-bound carbon was not detected due to quadrupolar relaxation. HRMS (APCI) $m / z$ calcd for $\mathrm{C}_{24} \mathrm{H}_{25} \mathrm{BN}_{2} \mathrm{OSi}$, [M-H] ${ }^{+}$ 397.1907. Found:397.1909. 
(Z)-2-(1-(3-methoxyphenyl)-4-(trimethylsilyl)but-1-en-3-yn-2-yl)-2,3-dihydro-1H-naphtho[1,8de] $[1,3,2]$ diazaborinine $(2 \mathrm{~d})$

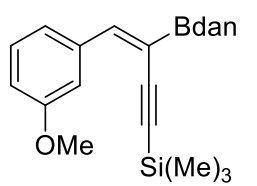

The titled compound was isolated $(74.3 \mathrm{mg}, 75 \%, 98: 2)$ as yellow oil after chromatography on silica gel (50:1 hexane/EtOAc). ${ }^{1} \mathrm{H}$ NMR $\left(500 \mathrm{MHz}, \mathrm{CDCl}_{3}\right) \delta 8.67(\mathrm{~d}, J=7.8 \mathrm{~Hz}, 1 \mathrm{H})$, $7.59(\mathrm{~s}, 1 \mathrm{H}), 7.36(\mathrm{t}, J=7.8 \mathrm{~Hz}, 1 \mathrm{H}), 7.17-7.13(\mathrm{~m}, 2 \mathrm{H}), 7.06(\mathrm{~d}, J=8.3 \mathrm{~Hz}, 2 \mathrm{H}), 7.01(\mathrm{t}, J$ $=7.6 \mathrm{~Hz}, 1 \mathrm{H}), 6.93(\mathrm{~d}, J=8.3 \mathrm{~Hz}, 1 \mathrm{H}), 6.41(\mathrm{~d}, J=7.3 \mathrm{~Hz}, 2 \mathrm{H}), 6.12(\mathrm{~s}, 2 \mathrm{H}), 3.91(\mathrm{~s}, 3 \mathrm{H})$, $0.32(\mathrm{~s}, 9 \mathrm{H}) .{ }^{13} \mathrm{C}\{\mathrm{H}\} \mathrm{NMR}\left(126 \mathrm{MHz}, \mathrm{CDCl}_{3}\right) \delta 157.4,141.1,139.0,136.5,130.9,129.3$, $127.7,125.7,120.1,120.0,117.8,110.6,106.1,106.0,104.3,55.7,0.2$. The boron-bound carbon was not detected due to quadrupolar relaxation. LRMS (EI) $m / z$ calcd for $\mathrm{C}_{24} \mathrm{H}_{25} \mathrm{BN}_{2} \mathrm{OSi}$, 396.18. Found:396.00.

(Z)-2-(1-(4-methoxyphenyl)-4-(trimethylsilyl)but-1-en-3-yn-2-yl)-2,3-dihydro-1H-naphtho[1,8de][1,3,2] diazaborinine (2e)<smiles>COc1ccc(/C=C(\C#C[SiH2])Cc2ccccc2)cc1</smiles>

The titled compound was isolated $(74.7 \mathrm{mg}, 75 \%$, >99:-) as yellow oil after chromatography on silica gel (50:1 hexane/EtOAc). ${ }^{1} \mathrm{H}$ NMR $\left(500 \mathrm{MHz}, \mathrm{CDCl}_{3}\right) \delta 8.07$ $(\mathrm{d}, J=8.8 \mathrm{~Hz}, 2 \mathrm{H}), 7.17-7.13(\mathrm{~m}, 2 \mathrm{H}), 7.06(\mathrm{~d}, J=8.2 \mathrm{~Hz}, 2 \mathrm{H}), 7.02(\mathrm{~s}, 1 \mathrm{H}), 6.94(\mathrm{~d}$, $J=8.8 \mathrm{~Hz}, 2 \mathrm{H}), 6.40(\mathrm{~d}, J=7.3 \mathrm{~Hz}, 2 \mathrm{H}), 6.06(\mathrm{~s}, 2 \mathrm{H}), 3.87(\mathrm{~s}, 3 \mathrm{H}), 0.36(\mathrm{~s}, 9 \mathrm{H}) .{ }^{13} \mathrm{C}\{\mathrm{H}\}$ NMR $\left(126 \mathrm{MHz} \mathrm{CDCl}_{3}\right) \delta 160.6,144.5,141.1,136.5,131.3,130.0,127.7,120.0,117.9$,

113.7, 106.1 (2 peaks overlap), 104.2, 55.5, 0.2. The boron-bound carbon was not detected due to quadrupolar relaxation. HRMS (APCI) $m / z$ calcd for $\mathrm{C}_{24} \mathrm{H}_{25} \mathrm{BN}_{2} \mathrm{OSi}$, [M-H] $]^{+}$397.1907. Found:397.1912.

\section{(Z)-4-(2-(1H-naphtho[1,8-de][1,3,2] diazaborinin-2(3H)-yl)-4-(trimethylsilyl)but-1-en-3-yn-1-yl)-N,N-} dimethylaniline (2f)<smiles>C[SiH2]C#C/C(=C\c1ccc(N(C)C)cc1)C(=O)Br</smiles>

The titled compound was isolated $(90.0 \mathrm{mg}, 88 \%,>99:-)$ as yellow solid after chromatography on silica gel (50:1 hexane/EtOAc). ${ }^{1} \mathrm{H}$ NMR $\left(500 \mathrm{MHz}, \mathrm{CDCl}_{3}\right) \delta 8.06$ $(\mathrm{d}, J=8.9 \mathrm{~Hz}, 2 \mathrm{H}), 7.19-7.14(\mathrm{~m}, 2 \mathrm{H}), 7.07(\mathrm{~d}, J=8.0 \mathrm{~Hz}, 2 \mathrm{H}), 7.00(\mathrm{~s}, 1 \mathrm{H}), 6.71(\mathrm{~d}$, $J=9.0 \mathrm{~Hz}, 2 \mathrm{H}), 6.41(\mathrm{~d}, J=7.3 \mathrm{~Hz}, 2 \mathrm{H}), 6.08(\mathrm{~s}, 2 \mathrm{H}), 3.04(\mathrm{~s}, 6 \mathrm{H}), 0.39(\mathrm{~s}, 9 \mathrm{H}) .{ }^{13} \mathrm{C}\{\mathrm{H}\}$ NMR $\left(126 \mathrm{MHz}, \mathrm{CDCl}_{3}\right) \delta 151.1,145.4,141.3,136.5,131.4,127.7,125.4,119.8$, 117.6, 111.3, 107.1, 106.0, 103.2, 40.2, 0.3. The boron-bound carbon was not detected due to quadrupolar relaxation. LRMS (EI) $m / z$ calcd for $\mathrm{C}_{25} \mathrm{H}_{28} \mathrm{BN}_{3} \mathrm{Si}$, 409.21. Found:409.00.

(Z)-2-(1-(4-fluorophenyl)-4-(trimethylsilyl)but-1-en-3-yn-2-yl)-2,3-dihydro-1H-naphtho[1,8de][1,3,2]diazaborinine $(2 \mathrm{~g})$<smiles>C[SiH2]C#C/C(=C\c1ccc(F)cc1)Cc1ccccc1</smiles>

The titled compound was isolated $(51.0 \mathrm{mg}, 53 \%, 97: 3)$ as yellow oil after chromatography on silica gel (100:1 hexane/EtOAc). ${ }^{1} \mathrm{H}$ NMR $\left(500 \mathrm{MHz}, \mathrm{CDCl}_{3}\right) \delta 8.07(\mathrm{dd}, J=8.6,5.6$ $\mathrm{Hz}, 2 \mathrm{H}), 7.16-7.12(\mathrm{~m}, 2 \mathrm{H}), 7.11-7.03(\mathrm{~m}, 5 \mathrm{H}), 6.40(\mathrm{~d}, J=7.2 \mathrm{~Hz}, 2 \mathrm{H}), 6.04(\mathrm{~s}, 2 \mathrm{H})$, $0.34(\mathrm{~s}, 9 \mathrm{H}) .{ }^{13} \mathrm{C}\{\mathrm{H}\} \mathrm{NMR}\left(126 \mathrm{MHz}, \mathrm{CDCl}_{3}\right) \delta 163.2(\mathrm{~d}, J=250.9 \mathrm{~Hz}), 143.5,140.9$, $136.5,133.2(\mathrm{~d}, J=3.2 \mathrm{~Hz}), 131.5(\mathrm{~d}, J=8.2 \mathrm{~Hz}), 127.7,120.1,118.1,115.4(\mathrm{~d}, J=21.5$ $\mathrm{Hz}), 106.3,105.4,105.1,0.1$. The boron-bound carbon was not detected due to quadrupolar relaxation. ${ }^{19} \mathrm{~F} \mathrm{NMR}$ (377 $\left.\mathrm{MHz}, \mathrm{CDCl}_{3}\right) \delta$-110.2. HRMS (APCI) $m / z$ calcd for $\mathrm{C}_{23} \mathrm{H}_{22} \mathrm{BFN}_{2} \mathrm{Si}$, [M-H] ${ }^{+}$385.1708. Found:385.1715

(Z)-2-(1-(4-chlorophenyl)-4-(trimethylsilyl)but-1-en-3-yn-2-yl)-2,3-dihydro-1H-naphtho[1,8de][1,3,2]diazaborinine $(2 \mathrm{~h})$<smiles>CSC#C/C(=C\c1ccc(Cl)cc1)CBr</smiles>

The titled compound was isolated $(71.0 \mathrm{mg}, 71 \%, 96: 4)$ as yellow oil after chromatography on silica gel (100:1 hexane/EtOAc). ${ }^{1} \mathrm{H}$ NMR $\left(500 \mathrm{MHz}, \mathrm{CDCl}_{3}\right) \delta 8.00$ $(\mathrm{d}, J=8.4 \mathrm{~Hz}, 2 \mathrm{H}), 7.37(\mathrm{~d}, J=8.3 \mathrm{~Hz}, 2 \mathrm{H}), 7.16-7.11(\mathrm{~m}, 2 \mathrm{H}), 7.08-7.01(\mathrm{~m}, 3 \mathrm{H})$, $6.39(\mathrm{~d}, J=7.2 \mathrm{~Hz}, 2 \mathrm{H}), 6.04(\mathrm{~s}, 2 \mathrm{H}), 0.34(\mathrm{~s}, 9 \mathrm{H}) .{ }^{13} \mathrm{C}\{\mathrm{H}\} \mathrm{NMR}\left(126 \mathrm{MHz}, \mathrm{CDCl}_{3}\right) \delta$ $143.3,140.9,136.5,135.3,135.1,130.8,128.6,127.7,120.1,118.1,106.3,106.0,105.3$, 0.1. The boron-bound carbon was not detected due to quadrupolar relaxation. HRMS (APCI) $\mathrm{m} / z$ calcd for $\mathrm{C}_{23} \mathrm{H}_{22} \mathrm{BClN}_{2} \mathrm{Si},[\mathrm{M}-\mathrm{H}]^{+}$401.1412. Found:401.1416. 
(Z)-2-(1-(4-bromophenyl)-4-(trimethylsilyl)but-1-en-3-yn-2-yl)-2,3-dihydro-1H-naphtho[1,8de][1,3,2] diazaborinine $(2 \mathrm{i})$<smiles>CSC#C/C(=C\c1ccc(Br)cc1)C(C)(C)C</smiles>

The titled compound was isolated $(57.7 \mathrm{mg}, 52 \%, 91: 9)$ as yellow oil after chromatography on silica gel (100:1 hexane/EtOAc). ${ }^{1} \mathrm{H}$ NMR $\left(500 \mathrm{MHz}, \mathrm{CDCl}_{3}\right) \delta 7.93$ $(\mathrm{d}, J=8.6 \mathrm{~Hz}, 2 \mathrm{H}), 7.53(\mathrm{~d}, J=8.5 \mathrm{~Hz}, 2 \mathrm{H}), 7.15-7.11(\mathrm{~m}, 2 \mathrm{H}), 7.05(\mathrm{~d}, J=8.2 \mathrm{~Hz}, 2 \mathrm{H})$, $7.01(\mathrm{~s}, 1 \mathrm{H}), 6.39(\mathrm{~d}, J=7.3 \mathrm{~Hz}, 2 \mathrm{H}), 6.03(\mathrm{~s}, 2 \mathrm{H}), 0.33(\mathrm{~s}, 9 \mathrm{H}) .{ }^{13} \mathrm{C}\{\mathrm{H}\} \mathrm{NMR}(126 \mathrm{MHz}$, $\left.\mathrm{CDCl}_{3}\right) \delta 143.3,140.9,136.5,135.8,131.6,131.0,127.7,123.5,120.1,118.2,106.3$, 106.2 , 105.2, 0.1. The boron-bound carbon was not detected due to quadrupolar relaxation. HRMS (APCI) $m / z$ calcd for $\mathrm{C}_{23} \mathrm{H}_{22} \mathrm{BBrN}_{2} \mathrm{Si}$, [M-H] $]^{+}$445.0907. Found:445.0907.

(Z)-2-(1-(4-(2-methyl-1,3-dioxolan-2-yl)phenyl)-4-(trimethylsilyl)but-1-en-3-yn-2-yl)-2,3-dihydro-1Hnaphtho[1,8-de][1,3,2]diazaborinine $(2 \mathrm{j})$<smiles>[M]C1(c2ccc(/C=C(\C#C[SiH2]C)C(=O)c3ccccc3)cc2)OCCO1</smiles>

The titled compound was isolated $(86.9 \mathrm{mg}, 77 \%$, >99:-) as yellow oil after chromatography on silica gel (30:1 hexane/EtOAc). ${ }^{1} \mathrm{H}$ NMR $\left(500 \mathrm{MHz}, \mathrm{CDCl}_{3}\right) \delta 8.07$ $(\mathrm{d}, J=8.3 \mathrm{~Hz}, 2 \mathrm{H}), 7.54(\mathrm{~d}, J=8.3 \mathrm{~Hz}, 2 \mathrm{H}), 7.15(\mathrm{t}, J=7.8 \mathrm{~Hz}, 2 \mathrm{H}), 7.09-7.04(\mathrm{~m}$, $3 \mathrm{H}), 6.40(\mathrm{~d}, J=7.3 \mathrm{~Hz}, 2 \mathrm{H}), 6.08(\mathrm{~s}, 2 \mathrm{H}), 4.08(\mathrm{t}, J=6.9 \mathrm{~Hz}, 2 \mathrm{H}), 3.82(\mathrm{t}, J=6.9 \mathrm{~Hz}$, $2 \mathrm{H}), 1.71(\mathrm{~s}, 3 \mathrm{H}), 0.36(\mathrm{~s}, 9 \mathrm{H}) .{ }^{13} \mathrm{C}\{\mathrm{H}\} \mathrm{NMR}\left(126 \mathrm{MHz}, \mathrm{CDCl}_{3}\right) \delta 144.7,144.3,140.9$, $136.5,136.4,129.5,127.7,125.4,120.0,118.0,108.8,106.2,105.6,105.4,64.6,27.6,0.1$. The boron-bound carbon was not detected due to quadrupolar relaxation. HRMS (APCI) $m / z$ calcd for $\mathrm{C}_{27} \mathrm{H}_{29} \mathrm{BN}_{2} \mathrm{O}_{2} \mathrm{Si},[\mathrm{M}-\mathrm{H}]^{+}$ 453.2170. Found:453.2180.

(Z)-4-(2-(1H-naphtho[1,8-de][1,3,2] diazaborinin-2(3H)-yl)-4-(trimethylsilyl)but-1-en-3-yn-1-yl)phenyl acetate $(2 \mathbf{k})$<smiles>CC(=O)Oc1ccc(C=C(C=C(C)C)C(C)C)cc1</smiles>

The titled compound was isolated $(60.3 \mathrm{mg}, 57 \%,>99:-)$ as yellow solid after chromatography on silica gel (30:1 hexane/EtOAc). ${ }^{1} \mathrm{H}$ NMR $\left(500 \mathrm{MHz}, \mathrm{CDCl}_{3}\right) \delta 8.09$ $(\mathrm{d}, J=8.6 \mathrm{~Hz}, 2 \mathrm{H}), 7.17-7.12(\mathrm{~m}, 4 \mathrm{H}), 7.05(\mathrm{~d}, J=7.8 \mathrm{~Hz}, 3 \mathrm{H}), 6.40(\mathrm{~d}, J=7.3 \mathrm{~Hz}$, 2H), $6.06(\mathrm{~s}, 2 \mathrm{H}), 2.33(\mathrm{~s}, 3 \mathrm{H}), 0.33(\mathrm{~s}, 9 \mathrm{H}) .{ }^{13} \mathrm{C}\{\mathrm{H}\} \mathrm{NMR}\left(126 \mathrm{MHz}, \mathrm{CDCl}_{3}\right) \delta 169.4$, $151.3,143.6,140.9,136.5,134.6,130.8,127.7,121.5,120.0,118.0,106.2,105.4(2$ peaks overlap), 21.3, 0.1. The boron-bound carbon was not detected due to quadrupolar relaxation. HRMS (APCI) $m / z$ calcd for $\mathrm{C}_{25} \mathrm{H}_{25} \mathrm{BN}_{2} \mathrm{O}_{2} \mathrm{Si}$, [M-H] $]^{+}$425.1857. Found:425.1871.

(Z)-2-(1-(6-methoxynaphthalen-2-yl)-4-(trimethylsilyl)but-1-en-3-yn-2-yl)-2,3-dihydro-1H-naphtho[1,8de][1,3,2] diazaborinine (2l)<smiles>COc1ccc2cc(/C=C(\C#C[SiH2]C)Cc3ccccc3)ccc2c1</smiles>

The titled compound was isolated $(93.0 \mathrm{mg}, 83 \%,>99:-)$ as yellow solid after chromatography on silica gel (30:1 hexane/EtOAc). ${ }^{1} \mathrm{H} \mathrm{NMR}\left(500 \mathrm{MHz}, \mathrm{CDCl}_{3}\right) \delta$ $8.47(\mathrm{~s}, 1 \mathrm{H}), 8.20(\mathrm{~d}, J=8.5 \mathrm{~Hz}, 1 \mathrm{H}), 7.75(\mathrm{t}, J=8.8 \mathrm{~Hz}, 2 \mathrm{H}), 7.20-7.13(\mathrm{~m}, 5 \mathrm{H})$, $7.08(\mathrm{~d}, J=8.2 \mathrm{~Hz}, 2 \mathrm{H}), 6.41(\mathrm{~d}, J=7.2 \mathrm{~Hz}, 2 \mathrm{H}), 6.09(\mathrm{~s}, 2 \mathrm{H}), 3.94(\mathrm{~s}, 3 \mathrm{H}), 0.41$ $(\mathrm{s}, 9 \mathrm{H}) .{ }^{13} \mathrm{C}\{\mathrm{H}\} \mathrm{NMR}\left(126 \mathrm{MHz}, \mathrm{CDCl}_{3}\right) \delta 158.8,145.0,141.0,136.5,135.3$, $132.5,130.3,129.6,128.7,127.7,127.4,126.7,120.0,119.3,117.9,106.2,106.0,105.9,104.8,55.4,0.3$. The boron-bound carbon was not detected due to quadrupolar relaxation. LRMS (EI) $m / z$ calcd for $\mathrm{C}_{28} \mathrm{H}_{27} \mathrm{BN}_{2} \mathrm{OSi}$, 446.19. Found:446.00.

(Z)-2-(1-(thiophen-3-yl)-4-(trimethylsilyl)but-1-en-3-yn-2-yl)-2,3-dihydro-1H-naphtho[1,8de] $[1,3,2]$ diazaborinine $(2 \mathrm{~m})$<smiles>CSC#C/C(=C\c1ccsc1)CBr</smiles>

The titled compound was isolated $(64.0 \mathrm{mg}, 69 \%,>99:-)$ as yellow oil after chromatography on silica gel (100:1 hexane/EtOAc). ${ }^{1} \mathrm{H}$ NMR $\left(500 \mathrm{MHz}, \mathrm{CDCl}_{3}\right) \delta 8.05(\mathrm{~d}, J=2.5 \mathrm{~Hz}, 1 \mathrm{H})$, $7.80(\mathrm{dd}, J=5.0,1.1 \mathrm{~Hz}, 1 \mathrm{H}), 7.34-7.32(\mathrm{~m}, 1 \mathrm{H}), 7.13(\mathrm{t}, J=7.8 \mathrm{~Hz}, 3 \mathrm{H}), 7.04(\mathrm{~d}, J=7.8$ $\mathrm{Hz}, 2 \mathrm{H}), 6.39(\mathrm{~d}, J=7.3 \mathrm{~Hz}, 2 \mathrm{H}), 6.03(\mathrm{~s}, 2 \mathrm{H}), 0.34(\mathrm{~s}, 9 \mathrm{H}) .{ }^{13} \mathrm{C}\{\mathrm{H}\} \mathrm{NMR}\left(126 \mathrm{MHz}, \mathrm{CDCl}_{3}\right)$ $\delta$ 141.0, 139.6, 138.4, 136.5, 128.5, 127.7, 125.4, 120.0, 118.0, 117.7, 106.2, 106.1, 105.9, 0.2. The boron-bound carbon was not detected due to quadrupolar relaxation. HRMS (APCI) $\mathrm{m} / z$ calcd for $\mathrm{C}_{21} \mathrm{H}_{21} \mathrm{BN}_{2} \mathrm{SSi}$, [M-H] ${ }^{+}$373.1366. Found:373.1366. 
$4 \mathrm{~mol} \%$ of $\mathrm{Co}(\mathrm{acac})_{2}$ and (E)-2,6-diisopropyl-N-(1-(6-(4-phenyl-4,5-dihydrooxazol-2yl)pyridin-2-yl)ethylidene)aniline was used to catalyze the reaction. The titled compound was isolated $(51.0 \mathrm{mg}, 63 \%, 90: 10)$ as a yellow oil after chromatography on silica gel $(50: 1$ hexane/EtOAc). ${ }^{1} \mathrm{H}$ NMR $\left(500 \mathrm{MHz}, \mathrm{CDCl}_{3}\right) \delta 7.14-7.10(\mathrm{~m}, 2 \mathrm{H}), 7.03(\mathrm{~d}, J=8.0 \mathrm{~Hz}$, $2 \mathrm{H}), 6.48(\mathrm{t}, J=7.2 \mathrm{~Hz}, 1 \mathrm{H}), 6.35(\mathrm{~d}, J=7.9 \mathrm{~Hz}, 2 \mathrm{H}), 5.92(\mathrm{~s}, 2 \mathrm{H}), 2.48(\mathrm{q}, J=7.3 \mathrm{~Hz}, 2 \mathrm{H})$, $1.54(\mathrm{q}, J=7.4 \mathrm{~Hz}, 2 \mathrm{H}), 0.99(\mathrm{t}, J=7.4 \mathrm{~Hz}, 3 \mathrm{H}), 0.29(\mathrm{~s}, 9 \mathrm{H}) .{ }^{13} \mathrm{C}\{\mathrm{H}\} \mathrm{NMR}(126 \mathrm{MHz}$, $\left.\mathrm{CDCl}_{3}\right) \delta 152.5,141.0,136.5,127.7,120.0,117.8,106.1,104.1,101.3,34.6,22.1,14.0,0.4$. The boron-bound carbon was not detected due to quadrupolar relaxation. LRMS (EI) $m / z$ calcd for $\mathrm{C}_{20} \mathrm{H}_{25} \mathrm{BN}_{2} \mathrm{Si}, 332.18$. Found: 332.00.

(Z)-2-(6-((tert-butyldimethylsilyl)oxy)-1-(trimethylsilyl)hex-3-en-1-yn-3-yl)-2,3-dihydro-1H-naphtho[1,8de][1,3,2] diazaborinine (2o)<smiles>CSC#C/C(=C\CCOC(C)(C)C)CBr</smiles>

$4 \mathrm{~mol} \%$ of $\mathrm{Co}(\mathrm{acac})_{2}$ and (E)-2,6-diisopropyl-N-(1-(6-(4-phenyl-4,5-dihydrooxazol-2yl)pyridin-2-yl)ethylidene)aniline was used to catalyze the reaction. The titled compound was isolated $(68.0 \mathrm{mg}, 61 \%, 93: 7)$ as colorless oil after chromatography on silica gel $(50: 1$ hexane/EtOAc). ${ }^{1} \mathrm{H}$ NMR $\left(500 \mathrm{MHz}, \mathrm{CDCl}_{3}\right) \delta 7.11(\mathrm{t}, J=7.8 \mathrm{~Hz}, 2 \mathrm{H}), 7.03(\mathrm{~d}, J=8.3 \mathrm{~Hz}, 2 \mathrm{H}), 6.53(\mathrm{t}, J=7.0$ $\mathrm{Hz}, 1 \mathrm{H}), 6.35(\mathrm{~d}, J=7.3 \mathrm{~Hz}, 2 \mathrm{H}), 5.91(\mathrm{~s}, 2 \mathrm{H}), 3.77(\mathrm{t}, J=6.6 \mathrm{~Hz}, 2 \mathrm{H}), 2.72(\mathrm{q}, J=6.8 \mathrm{~Hz}, 2 \mathrm{H}), 0.93(\mathrm{~s}, 9 \mathrm{H})$, $0.27(\mathrm{~s}, 9 \mathrm{H}), 0.10(\mathrm{~s}, 6 \mathrm{H}) .{ }^{13} \mathrm{C}\{\mathrm{H}\} \mathrm{NMR}\left(126 \mathrm{MHz}, \mathrm{CDCl}_{3}\right) \delta 148.6,141.0,136.5,127.7,120.0,117.9,106.1$, $103.8,101.7,62.0,36.3,26.1,18.5,0.4,-5.0$. The boron-bound carbon was not detected due to quadrupolar relaxation. LRMS (EI) $m / z$ calcd for $\mathrm{C}_{25} \mathrm{H}_{37} \mathrm{BN}_{2} \mathrm{OSi}_{2}$, [M] $]^{+} 448.25$. Found:448.00.

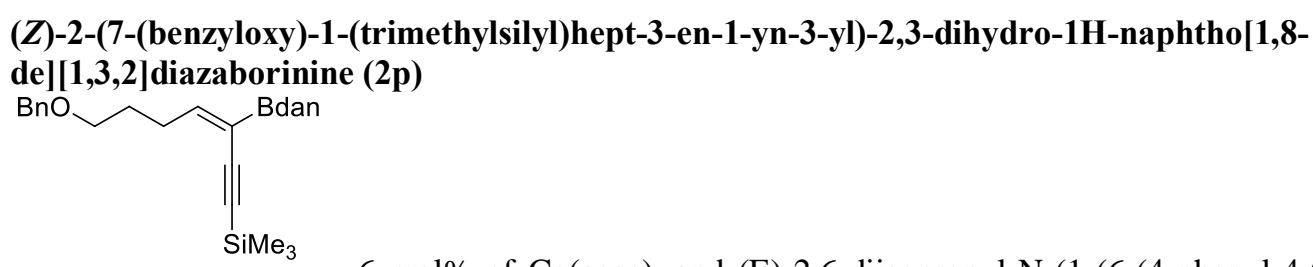

$6 \mathrm{~mol} \%$ of $\mathrm{Co}(\mathrm{acac})_{2}$ and (E)-2,6-diisopropyl-N-(1-(6-(4-phenyl-4,5-dihydrooxazol-2yl)pyridin-2-yl)ethylidene)aniline was used to catalyze the reaction. The titled compound was isolated (64.8 $\mathrm{mg}$, $59 \%, 91: 9)$ as brown oil after chromatography on silica gel $\left(50: 1\right.$ hexane/EtOAc). ${ }^{1} \mathrm{H} \mathrm{NMR}\left(500 \mathrm{MHz}, \mathrm{CDCl}_{3}\right) \delta$ $7.40-7.35(\mathrm{~m}, 4 \mathrm{H}), 7.32-7.28(\mathrm{~m}, 1 \mathrm{H}), 7.16-7.10(\mathrm{~m}, 2 \mathrm{H}), 7.04(\mathrm{~d}, J=8.2 \mathrm{~Hz}, 2 \mathrm{H}), 6.49(\mathrm{t}, J=7.1 \mathrm{~Hz}, 1 \mathrm{H})$, $6.35(\mathrm{~d}, J=7.2 \mathrm{~Hz}, 2 \mathrm{H}), 5.91(\mathrm{~s}, 2 \mathrm{H}), 4.55(\mathrm{~s}, 2 \mathrm{H}), 3.56(\mathrm{t}, J=6.4 \mathrm{~Hz}, 2 \mathrm{H}), 2.62(\mathrm{q}, J=7.3 \mathrm{~Hz}, 2 \mathrm{H}), 1.88-1.82$ $(\mathrm{m}, 2 \mathrm{H}), 0.29(\mathrm{~s}, 9 \mathrm{H}) .{ }^{13} \mathrm{C}\{\mathrm{H}\} \mathrm{NMR}\left(126 \mathrm{MHz}, \mathrm{CDCl}_{3}\right) \delta 151.9,141.0,138.6,136.4,128.5,127.8,127.7$ (2 peaks overlap), 120.0, 117.8, 106.1, 103.8, 101.7, 73.1, 70.0, 29.5, 28.8, 0.4. The boron-bound carbon was not detected due to quadrupolar relaxation. LRMS (EI) $m / z$ calcd for $\mathrm{C}_{27} \mathrm{H}_{31} \mathrm{BN}_{2} \mathrm{OSi}$, [M] $]^{+}$438.22. Found:438.00.

(Z)-2-(8-chloro-1-(trimethylsilyl)oct-3-en-1-yn-3-yl)-2,3-dihydro-1H-naphtho[1,8-de][1,3,2]diazaborinine (2q)<smiles>CSC#C/C(=C\CCCCCl)CBr</smiles>

$6 \mathrm{~mol} \%$ of $\mathrm{Co}(\mathrm{acac})_{2}$ and (E)-2,6-diisopropyl-N-(1-(6-(4-phenyl-4,5-dihydrooxazol2-yl)pyridin-2-yl)ethylidene)aniline was used to catalyze the reaction. The titled compound was isolated $(64.3 \mathrm{mg}, 68 \%, 95: 5)$ as yellow oil after chromatography on silica gel (50:1 hexane/EtOAc. ${ }^{1} \mathrm{H}$ NMR $\left(500 \mathrm{MHz}, \mathrm{CDCl}_{3}\right) \delta 7.11(\mathrm{t}, J=7.8 \mathrm{~Hz}, 2 \mathrm{H}), 7.03(\mathrm{~d}, J=8.2 \mathrm{~Hz}, 2 \mathrm{H}), 6.45(\mathrm{~d}, J=7.2 \mathrm{~Hz}, 1 \mathrm{H}), 6.35(\mathrm{~d}$, $J=7.2 \mathrm{~Hz}, 2 \mathrm{H}), 5.90(\mathrm{~s}, 2 \mathrm{H}), 3.60(\mathrm{t}, J=6.6 \mathrm{~Hz}, 2 \mathrm{H}), 2.53(\mathrm{q}, J=7.3 \mathrm{~Hz}, 2 \mathrm{H}), 1.86-1.82(\mathrm{~m}, 2 \mathrm{H}), 1.70-1.65$ $(\mathrm{m}, 2 \mathrm{H}), 0.28(\mathrm{~s}, 9 \mathrm{H}) .{ }^{13} \mathrm{C}\{\mathrm{H}\} \mathrm{NMR}\left(126 \mathrm{MHz}, \mathrm{CDCl}_{3}\right) \delta 151.3,140.9,136.5,127.7,120.0,117.9,106.1,103.7$, $101.8,44.9,32.0,31.5,25.8,0.4$. The boron-bound carbon was not detected due to quadrupolar relaxation. LRMS (EI) $m / z$ calcd for $\mathrm{C}_{21} \mathrm{H}_{26} \mathrm{BClN} 2 \mathrm{Si},[\mathrm{M}]^{+} 380.16$. Found:380.00. 
(Z)-2-(1,4-bis(4-methoxyphenyl)but-1-en-3-yn-2-yl)-2,3-dihydro-1H-naphtho[1,8-de][1,3,2]diazaborinine (2r)<smiles>COc1ccc(C#C/C(=C\c2ccc(OC)cc2)Cc2ccccc2)cc1</smiles>

The titled compound was isolated $(69.8 \mathrm{mg}, 65 \%,>99:-)$ as brown solid after chromatography on silica gel (50:1 hexane/EtOAc). ${ }^{1} \mathrm{H}$ NMR $\left(500 \mathrm{MHz}, \mathrm{CDCl}_{3}\right) \delta 8.06$ $(\mathrm{d}, J=8.8 \mathrm{~Hz}, 2 \mathrm{H}), 7.50(\mathrm{~d}, J=8.8 \mathrm{~Hz}, 2 \mathrm{H}), 7.14(\mathrm{t}, J=7.8 \mathrm{~Hz}, 2 \mathrm{H}), 7.05(\mathrm{~s}, 3 \mathrm{H}), 6.97$ - $6.92(\mathrm{~m}, 4 \mathrm{H}), 6.42(\mathrm{~d}, J=7.0 \mathrm{~Hz}, 2 \mathrm{H}), 6.11(\mathrm{~s}, 2 \mathrm{H}), 3.86(\mathrm{~s}, 6 \mathrm{H}) .{ }^{13} \mathrm{C}\{\mathrm{H}\} \mathrm{NMR}(126$ $\left.\mathrm{MHz}, \mathrm{CDCl}_{3}\right) \delta 160.4,159.9,142.8,141.2,133.1,131.2,130.3,127.7,120.0,117.9$, 116.1, 114.4, 114.2, 113.9, 106.2, 98.4, 88.8, 55.5 (2 peaks overlap). The boron-bound carbon was not detected due to quadrupolar relaxation. HRMS (APCI) $\mathrm{m} / \mathrm{z}$ calcd for $\mathrm{C}_{28} \mathrm{H}_{23} \mathrm{BN}_{2} \mathrm{O}_{2}$, [M-H] $]^{+}$431.1931. Found:431.1934.

(Z)-2-(1,4-bis(4-ethylphenyl)but-1-en-3-yn-2-yl)-2,3-dihydro-1H-naphtho[1,8-de][1,3,2]diazaborinine (2s)<smiles>CCc1ccc(C#C/C(=C\c2ccc(CC)cc2)Cc2ccccc2)cc1</smiles>

Found:427.2355.

The titled compound was isolated $(69.7 \mathrm{mg}, 66 \%,>99:-)$ as yellow solid after chromatography on silica gel (100:1 hexane/EtOAc). ${ }^{1} \mathrm{H}$ NMR $\left(500 \mathrm{MHz}, \mathrm{CDCl}_{3}\right) \delta 8.03$ $(\mathrm{d}, J=8.0 \mathrm{~Hz}, 2 \mathrm{H}), 7.50(\mathrm{~d}, J=7.8 \mathrm{~Hz}, 2 \mathrm{H}), 7.28-7.26(\mathrm{~m}, 3 \mathrm{H}), 7.15(\mathrm{t}, J=7.8 \mathrm{~Hz}, 3 \mathrm{H})$, $7.11(\mathrm{~s}, 1 \mathrm{H}), 7.06(\mathrm{~d}, J=8.3 \mathrm{~Hz}, 2 \mathrm{H}), 6.43(\mathrm{~d}, J=7.3 \mathrm{~Hz}, 2 \mathrm{H}), 6.13(\mathrm{~s}, 2 \mathrm{H}), 2.73-2.69$ $(\mathrm{m}, 4 \mathrm{H}), 1.30-1.27(\mathrm{~m}, 6 \mathrm{H}) .{ }^{13} \mathrm{C}\{\mathrm{H}\} \mathrm{NMR}\left(126 \mathrm{MHz}, \mathrm{CDCl}_{3}\right) \delta 145.9,145.1,143.6$, $141.1,136.5,134.7,131.6,129.6,128.3,128.1,127.7,121.0,120.0,117.9,106.2,99.0$, 89.3, 29.0 (2 peaks overlap),15.6, 15.5. The boron-bound carbon was not detected due to quadrupolar relaxation. HRMS (APCI) $m / z$ calcd for $\mathrm{C}_{30} \mathrm{H}_{27} \mathrm{BN}_{2},[\mathrm{M}-\mathrm{H}]^{+} 427.2346$.

(Z)-2-(1,4-diphenylbut-1-en-3-yn-1-yl)-4,4,5,5-tetramethyl-1,3,2-dioxaborolane (not in maintext)<smiles>C(#Cc1ccccc1)/C=C\c1ccccc1</smiles>

The titled compound was isolated $(58.0 \mathrm{mg}, 70 \%, 98: 2)$ as brown oil after chromatography on silica gel (100:1 hexane/EtOAc). ${ }^{1} \mathrm{H}$ NMR $\left(400 \mathrm{MHz}, \mathrm{CDCl}_{3}\right) \delta 8.09(\mathrm{~d}, J=7.2 \mathrm{~Hz}, 2 \mathrm{H}), 7.54$ $(\mathrm{dd}, J=7.9,1.6 \mathrm{~Hz}, 2 \mathrm{H}), 7.45(\mathrm{~s}, 1 \mathrm{H}), 7.41(\mathrm{t}, J=7.3 \mathrm{~Hz}, 2 \mathrm{H}), 7.37-7.32(\mathrm{~m}, 4 \mathrm{H}), 1.36(\mathrm{~s}$, $12 \mathrm{H}) .{ }^{13} \mathrm{C}\{\mathrm{H}\}$ NMR $\left(101 \mathrm{MHz}, \mathrm{CDCl}_{3}\right) \delta 149.7,137.2,131.8,129.8,129.4,128.4$ (2 peaks overlap), 128.1, 124.4, 97.5, 90.2, 84.4, 25.0. The boron-bound carbon was not detected due to quadrupolar relaxation. LR-MS (EI) $\mathrm{m} / z$ calcd for $\mathrm{C}_{22} \mathrm{H}_{23} \mathrm{BO}_{2}, 330.17$. Found: 330.00. 


\section{General procedure for Co-catalyzed $\alpha$-hydroboration of 1,3-diynes}

In an Ar-filled glovebox, a mixture of Co(acac $)_{2}(0.6 \mathrm{mg}, 2.5 \mu \mathrm{mol})$ and dppf $(1.4 \mathrm{mg}, 2.5 \mu \mathrm{mol})$ in THF $(0.5 \mathrm{~mL})$ was added into a 4-mL screw-capped vial containing a magnetic stirring bar. The resulting mixture was stirred for 2 minutes before adding HBpin $(32.0 \mathrm{mg}, 0.250 \mathrm{mmol})$ and 1,3 -diynes $(0.250 \mathrm{mmol})$ successively. The reaction mixture was stirred at room temperature for 18 hours.

Note: As the Bpin derivatives are stable in column chromatography to a limited extent, most of the boronsubstituted enynes were further converted to its Bdan derivatives to facilitate the isolation process.

Upon reaction completion, $\mathrm{FeCl}_{3}(3.2 \mathrm{mg}, 20.0 \mu \mathrm{mol}), 1,8$-diaminonaphthalene ( $40.0 \mathrm{mg}, 0.250 \mathrm{mmol}$ ), imidazole (34.0 $\mathrm{mg}, 0.500 \mathrm{mmol}$ ) was added into another 4-mL screw-capped vial containing a magnetic stirring bar containing water $(0.5 \mathrm{~mL})$ and acetonitrile $(0.5 \mathrm{~mL})$. Subsequently, the crude hydroboration reaction mixture was transferred and rinsed with acetonitrile $(1.5 \mathrm{~mL})$ into the latter vial. It was then stirred for 12 to $18 \mathrm{hr}$ at room temperature for the conversion to Bdan derivatives to take place. ${ }^{3}$ After the completion of the reaction, the crude mixture was washed with saturated $\mathrm{NaCl}$ and extracted thrice with ethyl acetate. The organic layer was then dried over sodium sulfate, filtered and concentrated under vacuum. Lastly, the residue was purified by flash chromatography on silica using a mixture of ethyl acetate and hexane as eluent. The details for separation and characterization data of the products are stated below.

(Z)-2-(1-phenyl-4-(trimethylsilyl)but-1-en-3-yn-1-yl)-2,3-dihydro-1H-naphtho[1,8-de][1,3,2]diazaborinine (3a)<smiles>CC#C/C=C(\Br)c1ccccc1</smiles>

The titled compound was isolated $(67.7 \mathrm{mg}, 74 \%,-:>99)$ as yellow oil after chromatography on silica gel (100:1 hexane/EtOAc). ${ }^{1} \mathrm{H}$ NMR $\left(500 \mathrm{MHz}, \mathrm{CDCl}_{3}\right) \delta 7.51(\mathrm{~d}, J=7.1 \mathrm{~Hz}, 2 \mathrm{H})$, $7.40(\mathrm{t}, J=7.4 \mathrm{~Hz}, 2 \mathrm{H}), 7.35(\mathrm{~d}, J=7.2 \mathrm{~Hz}, 1 \mathrm{H}), 7.11-7.07(\mathrm{~m}, 2 \mathrm{H}), 7.03(\mathrm{~d}, J=8.3 \mathrm{~Hz}, 2 \mathrm{H})$, $6.28(\mathrm{~d}, J=7.3 \mathrm{~Hz}, 2 \mathrm{H}), 6.17(\mathrm{~s}, 1 \mathrm{H}), 5.68(\mathrm{~s}, 2 \mathrm{H}), 0.13(\mathrm{~s}, 9 \mathrm{H}) .{ }^{13} \mathrm{C}\{\mathrm{H}\} \mathrm{NMR}(126 \mathrm{MHz}$, $\mathrm{CDCl} 3) \delta 140.9,139.5,136.5,129.1,128.3,127.9,127.7,120.1,118.1,116.4,106.3,103.6$, 102.2, -0.2. The boron-bound carbon was not detected due to quadrupolar relaxation. HRMS (APCI) $\mathrm{m} / z$ calcd for $\mathrm{C}_{23} \mathrm{H}_{23} \mathrm{BN}_{2} \mathrm{Si}$, [M-H] $]^{+}$367.1802. Found: 367.1810.

\section{(Z)-2-(1-phenyl-4-(triethylsilyl)but-1-en-3-yn-1-yl)-2,3-dihydro-1H-naphtho[1,8-de][1,3,2]diazaborinine} (3b)<smiles>CCC#CC=C(c1ccccc1)c1ccccc1</smiles>

The titled compound was isolated ( $74.0 \mathrm{mg}, 72 \%,-:>99)$ as yellow oil after chromatography on silica gel (100:1 hexane/EtOAc). ${ }^{1} \mathrm{H}$ NMR $(500 \mathrm{MHz}, \mathrm{CDCl} 3) \delta 7.51(\mathrm{~d}, \mathrm{~J}=7.0 \mathrm{~Hz}, 2 \mathrm{H}), 7.39$ $(\mathrm{t}, \mathrm{J}=7.4 \mathrm{~Hz}, 2 \mathrm{H}), 7.34(\mathrm{~d}, \mathrm{~J}=7.2 \mathrm{~Hz}, 1 \mathrm{H}), 7.12-7.08(\mathrm{~m}, 2 \mathrm{H}), 7.03(\mathrm{~d}, \mathrm{~J}=8.2 \mathrm{~Hz}, 2 \mathrm{H}), 6.28$ $(\mathrm{d}, \mathrm{J}=7.2 \mathrm{~Hz}, 2 \mathrm{H}), 6.22(\mathrm{~s}, 1 \mathrm{H}), 5.69(\mathrm{~s}, 2 \mathrm{H}), 0.93(\mathrm{t}, \mathrm{J}=7.9 \mathrm{~Hz}, 6 \mathrm{H}), 0.57(\mathrm{q}, \mathrm{J}=7.9 \mathrm{~Hz}, 9 \mathrm{H})$. ${ }^{13} \mathrm{C}\{\mathrm{H}\}$ NMR $\left(126 \mathrm{MHz}, \mathrm{CDCl}_{3}\right) \delta 140.9,139.6,136.4,129.1,128.3,127.8,127.7,120.1$, $118.1,116.7,106.2,104.5,100.0,7.5,4.4$. The boron-bound carbon was not detected due to quadrupolar relaxation. HRMS (APCI) $m / z$ calcd for $\mathrm{C}_{26} \mathrm{H}_{29} \mathrm{BN}_{2} \mathrm{Si}$, [M-H] $]^{+}$409.2271. Found: 409.2275.

(Z)-2-(1-(2-methoxyphenyl)-4-(trimethylsilyl)but-1-en-3-yn-1-yl)-2,3-dihydro-1H-naphtho[1,8de][1,3,2]diazaborinine $(3 c)$<smiles>CC#C/C=C(/CCC)c1ccccc1OC</smiles>

The titled compound was isolated (93.1 mg, 94\%, -:>99) as yellow solid after chromatography on silica gel (50:1 hexane/EtOAc). ${ }^{1} \mathrm{H}$ NMR $\left(500 \mathrm{MHz}, \mathrm{CDCl}_{3}\right) \delta 7.53(\mathrm{~d}, J=7.5 \mathrm{~Hz}, 1 \mathrm{H})$, 7.34 (t, $J=7.8 \mathrm{~Hz}, 1 \mathrm{H}), 7.09$ (t, $J=7.7 \mathrm{~Hz}, 2 \mathrm{H}), 7.02$ (t, $J=7.3 \mathrm{~Hz}, 3 \mathrm{H}), 6.94$ (d, $J=8.3 \mathrm{~Hz}$, $1 \mathrm{H}), 6.26(\mathrm{~d}, J=7.3 \mathrm{~Hz}, 2 \mathrm{H}), 6.18-6.14(\mathrm{~m}, 1 \mathrm{H}), 5.66(\mathrm{~s}, 2 \mathrm{H}), 3.76(\mathrm{~s}, 3 \mathrm{H}), 0.11(\mathrm{~s}, 9 \mathrm{H})$. ${ }^{13} \mathrm{C}\{\mathrm{H}\} \mathrm{NMR}\left(126 \mathrm{MHz}, \mathrm{CDCl}_{3}\right) \delta 156.6,141.3,136.5,130.7,129.3,128.9,127.7,120.5$, $120.0,117.6,116.5,111.2,105.9,103.5,101.3,55.9,-0.2$. The boron-bound carbon was not detected due to quadrupolar relaxation. GC-MS(EI) $m / z$ calcd for $\mathrm{C}_{24} \mathrm{H}_{25} \mathrm{BN}_{2} \mathrm{OSi}$, $[\mathrm{M}-\mathrm{H}]^{+}$396.18. Found: 396.00 
(Z)-2-(1-(3-methoxyphenyl)-4-(trimethylsilyl)but-1-en-3-yn-1-yl)-2,3-dihydro-1H-naphtho[1,8de][1,3,2]diazaborinine $(3 \mathrm{~d})$<smiles>CC#C/C=C(\c1cccc(OC)c1)C(Br)(Br)c1ccccc1</smiles>

The titled compound was isolated $(60.0 \mathrm{mg}, 61 \%, 1: 99)$ as yellow solid after chromatography on silica gel (50:1 hexane/EtOAc). ${ }^{1} \mathrm{H}$ NMR $\left(500 \mathrm{MHz}, \mathrm{CDCl}_{3}\right) \delta 7.31$ $(\mathrm{t}, J=7.9 \mathrm{~Hz}, 1 \mathrm{H}), 7.12-7.07(\mathrm{~m}, 3 \mathrm{H}), 7.04(\mathrm{t}, J=8.5 \mathrm{~Hz}, 3 \mathrm{H}), 6.90(\mathrm{dd}, J=8.2,2.5$ $\mathrm{Hz}, 1 \mathrm{H}), 6.29$ (d, J=7.2 Hz, 2H), $6.18(\mathrm{~s}, 1 \mathrm{H}), 5.70(\mathrm{~s}, 2 \mathrm{H}), 3.84$ (s, 3H), $0.14(\mathrm{~s}, 9 \mathrm{H})$. ${ }^{13} \mathrm{C}\{\mathrm{H}\}$ NMR $\left(126 \mathrm{MHz}, \mathrm{CDCl}_{3}\right) \delta 159.6,140.9,140.8,136.4,129.3,127.7,121.5$, $120.1,118.1,116.5,114.3,113.9,106.3,103.5,102.5,55.4,-0.2$. The boron-bound carbon was not detected due to quadrupolar relaxation. HRMS (APCI) $m / z$ calcd for $\mathrm{C}_{24} \mathrm{H}_{25} \mathrm{BN}_{2} \mathrm{OSi}$, [M-H] 397.1907. Found: 397.1916.

(Z)-2-(1-(4-methoxyphenyl)-4-(trimethylsilyl)but-1-en-3-yn-1-yl)-2,3-dihydro-1H-naphtho[1,8de][1,3,2]diazaborinine $(3 \mathrm{e})$<smiles>CC#C/C=C(\c1ccc(OC)cc1)C(C)(C)C</smiles>

The titled compound was isolated $(66.0 \mathrm{mg}, 67 \%,-:>99)$ as yellow oil after chromatography on silica gel (50:1 hexane/EtOAc). ${ }^{1} \mathrm{H}$ NMR $\left(500 \mathrm{MHz}, \mathrm{CDCl}_{3}\right) \delta 7.55$ $(\mathrm{d}, J=8.8 \mathrm{~Hz}, 2 \mathrm{H}), 7.12-7.08(\mathrm{~m}, 2 \mathrm{H}), 7.04(\mathrm{~d}, J=8.3 \mathrm{~Hz}, 2 \mathrm{H}), 6.93(\mathrm{~d}, J=8.8 \mathrm{~Hz}$, $2 \mathrm{H}), 6.28(\mathrm{~d}, J=7.2 \mathrm{~Hz}, 2 \mathrm{H}), 6.09(\mathrm{~s}, 1 \mathrm{H}), 5.70(\mathrm{~s}, 2 \mathrm{H}), 3.86(\mathrm{~s}, 3 \mathrm{H}), 0.18(\mathrm{~s}, 9 \mathrm{H}) .{ }^{13} \mathrm{C}\{\mathrm{H}\}$ NMR $\left(126 \mathrm{MHz}, \mathrm{CDCl}_{3}\right) \delta 159.4,140.9,136.4,131.7,130.7,127.7,120.1,118.1,114.6$, 113.6, 106.2, 104.0, 101.7, 55.4, -0.2. The boron-bound carbon was not detected due to quadrupolar relaxation. HRMS (APCI) $m / z$ calcd for $\mathrm{C}_{24} \mathrm{H}_{25} \mathrm{BN}_{2} \mathrm{OSi}$, [M-H] $]^{+}$397.1907. Found: 397.1917 .

(Z)-4-(1-(1H-naphtho[1,8-de][1,3,2]diazaborinin-2(3H)-yl)-4-(trimethylsilyl)but-1-en-3-yn-1-yl)-N,Ndimethylaniline (3f)<smiles>C[SiH2]C#C/C=C(\Br)c1ccc(N(C)C)cc1</smiles>

The titled compound was isolated $(75.0 \mathrm{mg}, 73 \%, 8: 92)$ as yellow oil after chromatography on silica gel (30:1 hexane/EtOAc). ${ }^{1} \mathrm{H}$ NMR $\left(500 \mathrm{MHz}, \mathrm{CDCl}_{3}\right) \delta 7.61$ $(\mathrm{d}, J=8.9 \mathrm{~Hz}, 2 \mathrm{H}), 7.12-7.08(\mathrm{~m}, 2 \mathrm{H}), 7.03(\mathrm{~d}, J=8.2 \mathrm{~Hz}, 2 \mathrm{H}), 6.72(\mathrm{~d}, J=8.9 \mathrm{~Hz}$, 2H), $6.28(\mathrm{~d}, J=7.3 \mathrm{~Hz}, 2 \mathrm{H}), 5.98(\mathrm{~s}, 1 \mathrm{H}), 5.75(\mathrm{~s}, 2 \mathrm{H}), 3.02(\mathrm{~s}, 6 \mathrm{H}), 0.20(\mathrm{~s}, 9 \mathrm{H})$. ${ }^{13} \mathrm{C}\{\mathrm{H}\}$ NMR $\left(126 \mathrm{MHz}, \mathrm{CDCl}_{3}\right) \delta 150.3,141.1,136.5,130.7,127.7,127.1,120.1$, $117.9,112.0,111.7,106.1,104.9,101.0,40.5,-0.04$. The boron-bound carbon was not detected due to quadrupolar relaxation. HRMS (APCI) $\mathrm{m} / z$ calcd for $\mathrm{C}_{25} \mathrm{H}_{28} \mathrm{BN}_{3} \mathrm{OSi}$, $[\mathrm{M}-\mathrm{H}]^{+} 410.2224$. Found: 410.2227 .

(Z)-2-(1-(4-fluorophenyl)-4-(trimethylsilyl)but-1-en-3-yn-1-yl)-2,3-dihydro-1H-naphtho[1,8de][1,3,2]diazaborinine $(3 \mathrm{~g})$<smiles>COC#C/C=C(\Br)c1ccc(F)cc1</smiles>

The titled compound was isolated $(68.2 \mathrm{mg}, 71 \%, 5: 95)$ as yellow oil after chromatography on silica gel (100:1 hexane/EtOAc). ${ }^{1} \mathrm{H}$ NMR $\left(500 \mathrm{MHz}, \mathrm{CDCl}_{3}\right) \delta 7.53-7.48(\mathrm{~m}, 2 \mathrm{H})$, $7.12-7.06(\mathrm{~m}, 4 \mathrm{H}), 7.04(\mathrm{~d}, J=8.3 \mathrm{~Hz}, 2 \mathrm{H}), 6.29(\mathrm{~d}, J=7.2 \mathrm{~Hz}, 2 \mathrm{H}), 6.16(\mathrm{~s}, 1 \mathrm{H}), 5.64$ $(\mathrm{s}, 2 \mathrm{H}), 0.14(\mathrm{~s}, 9 \mathrm{H}) .{ }^{13} \mathrm{C}\{\mathrm{H}\} \mathrm{NMR}\left(126 \mathrm{MHz}, \mathrm{CDCl}_{3}\right) \delta 162.5\left(\mathrm{~d}, J_{\mathrm{C}-\mathrm{F}}=247.3 \mathrm{~Hz}\right), 140.8$, $136.5,135.3\left(\mathrm{~d}, J_{\mathrm{C}-\mathrm{F}}=3.4 \mathrm{~Hz}\right), 131.0\left(\mathrm{~d}, J_{\mathrm{C}-\mathrm{F}}=8.0 \mathrm{~Hz}\right), 127.7,120.1,118.3,116.5,115.2$ $\left(\mathrm{d}, J_{\mathrm{C}-\mathrm{F}}=21.4 \mathrm{~Hz}\right), 106.3,103.3,102.5,-0.2 .{ }^{19} \mathrm{~F} \mathrm{NMR}\left(377 \mathrm{MHz}, \mathrm{CDCl}_{3}\right) \delta-110.2$. The boron-bound carbon was not detected due to quadrupolar relaxation. HRMS (APCI) $m / z$ calcd for $\mathrm{C}_{23} \mathrm{H}_{22} \mathrm{BFN}_{2} \mathrm{Si}_{\text {, }}$ $[\mathrm{M}-\mathrm{H}]^{+}$385.1708. Found: 385.1713 .

(Z)-2-(1-(4-chlorophenyl)-4-(trimethylsilyl)but-1-en-3-yn-1-yl)-2,3-dihydro-1H-naphtho[1,8de][1,3,2] diazaborinine $(3 \mathrm{~h})$<smiles>CSC#C/C=C(\Br)c1ccc(Cl)cc1</smiles>

The titled compound was isolated $(67.0 \mathrm{mg}, 67 \%, 2: 98)$ as yellow oil after chromatography on silica gel (100:1 hexane/EtOAc). ${ }^{1} \mathrm{H}$ NMR $\left(500 \mathrm{MHz}, \mathrm{CDCl}_{3}\right) \delta 7.46(\mathrm{~d}, J=8.5 \mathrm{~Hz}$, $2 \mathrm{H}), 7.37(\mathrm{~d}, J=8.4 \mathrm{~Hz}, 2 \mathrm{H}), 7.12-7.08(\mathrm{~m}, 2 \mathrm{H}), 7.04(\mathrm{~d}, J=8.2 \mathrm{~Hz}, 2 \mathrm{H}), 6.29$ (d, $J=$ $7.2 \mathrm{~Hz}, 2 \mathrm{H}), 6.18(\mathrm{~s}, 1 \mathrm{H}), 5.63(\mathrm{~s}, 2 \mathrm{H}), 0.15(\mathrm{~s}, 9 \mathrm{H}) .{ }^{13} \mathrm{C}\{\mathrm{H}\} \mathrm{NMR}\left(126 \mathrm{MHz}, \mathrm{CDCl}_{3}\right) \delta$ $140.7,137.8,136.4,133.7,130.6,128.5,127.7,120.1,118.3,116.9,106.3,103.2$, 103.0, 0.2 . The boron-bound carbon was not detected due to quadrupolar relaxation. HRMS (APCI) $m / z$ calcd for $\mathrm{C}_{23} \mathrm{H}_{22} \mathrm{BClN}_{2} \mathrm{Si}$, [M-H] $]^{+}$401.1412. Found: 401.1417. 
(Z)-2-(1-(4-bromophenyl)-4-(trimethylsilyl)but-1-en-3-yn-1-yl)-2,3-dihydro-1H-naphtho[1,8de][1,3,2]diazaborinine $(3 \mathrm{i})$<smiles>CSC#C/C=C(\Br)c1ccc(Br)cc1</smiles>

The titled compound was isolated $(65.0 \mathrm{mg}, 58 \%,-:>99)$ as yellow oil after chromatography on silica gel (100:1 hexane/EtOAc). ${ }^{1} \mathrm{H}$ NMR $\left(500 \mathrm{MHz}, \mathrm{CDCl}_{3}\right) \delta 7.53$ $(\mathrm{d}, J=8.5 \mathrm{~Hz}, 2 \mathrm{H}), 7.40(\mathrm{~d}, J=8.4 \mathrm{~Hz}, 2 \mathrm{H}), 7.12-7.07(\mathrm{~m}, 2 \mathrm{H}), 7.04(\mathrm{~d}, J=8.2 \mathrm{~Hz}, 2 \mathrm{H})$, $6.29(\mathrm{~d}, J=7.2 \mathrm{~Hz}, 2 \mathrm{H}), 6.18(\mathrm{~s}, 1 \mathrm{H}), 5.63(\mathrm{~s}, 2 \mathrm{H}), 0.15(\mathrm{~s}, 9 \mathrm{H}) .{ }^{13} \mathrm{C}\{\mathrm{H}\} \mathrm{NMR}(126 \mathrm{MHz}$, $\left.\mathrm{CDCl}_{3}\right) \delta 140.7,138.3,136.4,131.5,130.9,127.7,121.9,120.1,118.3,116.9,106.4$, 103.2, 103.1, -0.2. The boron-bound carbon was not detected due to quadrupolar relaxation. HRMS (APCI) $m / z$ calcd for $\mathrm{C}_{23} \mathrm{H}_{22} \mathrm{BBrN}_{2} \mathrm{Si}$, [M-H] $]^{+}$445.0907. Found: 445.0919.

(Z)-2-(1-(4-(2-methyl-1,3-dioxolan-2-yl)phenyl)-4-(trimethylsilyl)but-1-en-3-yn-1-yl)-2,3-dihydro-1Hnaphtho[1,8-de][1,3,2]diazaborinine $(3 \mathbf{j})$<smiles>[M]C1(c2ccc(/C(=C/C#C[SiH2]C)CCCC)cc2)OCCO1</smiles>

The titled compound was isolated $(81.3 \mathrm{mg}, 72 \%, 1: 99)$ as yellow oil after chromatography on silica gel (30:1 hexane/EtOAc). ${ }^{1} \mathrm{H}$ NMR $\left(500 \mathrm{MHz}, \mathrm{CDCl}_{3}\right) \delta 7.51$ $(\mathrm{d}, J=1.1 \mathrm{~Hz}, 4 \mathrm{H}), 7.12-7.07(\mathrm{~m}, 2 \mathrm{H}), 7.03(\mathrm{~d}, J=7.7 \mathrm{~Hz}, 2 \mathrm{H}), 6.29(\mathrm{~d}, J=7.3 \mathrm{~Hz}$, $2 \mathrm{H}), 6.17(\mathrm{~s}, 1 \mathrm{H}), 5.70(\mathrm{~s}, 2 \mathrm{H}), 4.10-4.06(\mathrm{~m}, 2 \mathrm{H}), 3.86-3.82(\mathrm{~m}, 2 \mathrm{H}), 1.71(\mathrm{~s}, 3 \mathrm{H})$, $0.13(\mathrm{~s}, 9 \mathrm{H}) .{ }^{13} \mathrm{C}\{\mathrm{H}\} \mathrm{NMR}\left(126 \mathrm{MHz}, \mathrm{CDCl}_{3}\right) \delta 142.9,140.8,139.0,136.4,129.0,127.7$, $125.2,120.1,118.1,116.5,109.0,106.3,103.7,102.3,64.7,27.7,-0.3$. The boron-bound carbon was not detected due to quadrupolar relaxation. HRMS (APCI) $m / z$ calcd for $\mathrm{C}_{27} \mathrm{H}_{29} \mathrm{BN}_{2} \mathrm{O}_{2} \mathrm{Si}$, [M-H] 453.2170. Found: 453.2168 .

(Z)-4-(1-(1H-naphtho[1,8-de][1,3,2]diazaborinin-2(3H)-yl)-4-(trimethylsilyl)but-1-en-3-yn-1-yl)phenyl acetate $(3 \mathbf{k})$<smiles>CC(C)=O</smiles>

The titled compound was isolated $(58.9 \mathrm{mg}, 56 \%,-:>99)$ as yellow oil after chromatography on silica gel (50:1 hexane/EtOAc). ${ }^{1} \mathrm{H}$ NMR $\left(500 \mathrm{MHz}, \mathrm{CDCl}_{3}\right) \delta 7.56$ $(\mathrm{d}, J=8.6 \mathrm{~Hz}, 2 \mathrm{H}), 7.12(\mathrm{~d}, J=8.7 \mathrm{~Hz}, 2 \mathrm{H}), 7.09(\mathrm{~d}, J=7.3 \mathrm{~Hz}, 2 \mathrm{H}), 7.03(\mathrm{~d}, J=8.3$ $\mathrm{Hz}, 2 \mathrm{H}), 6.30$ (d, J=7.3 Hz, 2H), $6.17(\mathrm{~s}, 1 \mathrm{H}), 5.71(\mathrm{~s}, 2 \mathrm{H}), 2.34$ (s, 3H), $0.15(\mathrm{~s}, 9 \mathrm{H})$. ${ }^{13} \mathrm{C}\{\mathrm{H}\}$ NMR $\left(126 \mathrm{MHz}, \mathrm{CDCl}_{3}\right) \delta 169.7,150.3,140.8,136.9,136.4,130.4,127.7$, $121.4,120.1,118.2,116.5,106.3,103.4,102.6,21.3,-0.2$. The boron-bound carbon was not detected due to quadrupolar relaxation. HRMS (APCI) $m / z$ calcd for $\mathrm{C}_{25} \mathrm{H}_{25} \mathrm{BN}_{3} \mathrm{O}_{2} \mathrm{Si}$, [M-H] $]^{+} 425.1857$. Found: 425.1865 .

(Z)-2-(1-(6-methoxynaphthalen-2-yl)-4-(trimethylsilyl)but-1-en-3-yn-1-yl)-2,3-dihydro-1H-naphtho[1,8de][1,3,2] diazaborinine $(31)$<smiles>COc1ccc2cc(/C(=C\C#C[SiH2])C(=O)c3ccccc3)ccc2c1</smiles>

The titled compound was isolated $(79.0 \mathrm{mg}, 71 \%,-:>99)$ as yellow oil after chromatography on silica gel (30:1 hexane/EtOAc). ${ }^{1} \mathrm{H}$ NMR $\left(500 \mathrm{MHz}, \mathrm{CDCl}_{3}\right) \delta$ $7.98(\mathrm{~s}, 1 \mathrm{H}), 7.78(\mathrm{~d}, J=8.3 \mathrm{~Hz}, 2 \mathrm{H}), 7.70(\mathrm{dd}, J=8.5,1.7 \mathrm{~Hz}, 1 \mathrm{H}), 7.22-7.19$ $(\mathrm{m}, 2 \mathrm{H}), 7.12(\mathrm{~d}, J=7.3 \mathrm{~Hz}, 2 \mathrm{H}), 7.07(\mathrm{~d}, J=7.5 \mathrm{~Hz}, 2 \mathrm{H}), 6.30(\mathrm{dd}, J=7.2,0.9$ $\mathrm{Hz}, 2 \mathrm{H}), 6.24$ (s, 1H), $5.76(\mathrm{~s}, 2 \mathrm{H}), 3.98$ (s, 3H), 0.15 (s, 9H). ${ }^{13} \mathrm{C}\{\mathrm{H}\}$ NMR $(126$ $\left.\mathrm{MHz}_{1} \mathrm{CDCl}_{3}\right) \delta 158.2,140.9,136.5,134.6,134.3,129.8,128.8,128.3,127.9,127.7$, $126.5,120.1,119.1,118.1,115.8,106.3,105.8,103.9,102.1,55.5,-0.1$. The boron-bound carbon was not detected due to quadrupolar relaxation. HRMS (APCI) $m / z$ calcd for $\mathrm{C}_{28} \mathrm{H}_{27} \mathrm{BN}_{2} \mathrm{OSi}$, [M-H] $]^{+}$447.2064. Found: 447.2073.

(Z)-2-(1-(thiophen-3-yl)-4-(trimethylsilyl)but-1-en-3-yn-1-yl)-2,3-dihydro-1H-naphtho[1,8de $][1,3,2]$ diazaborinine $(3 \mathrm{~m})$

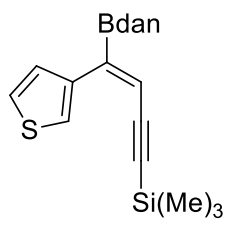

The titled compound was isolated $(65.1 \mathrm{mg}, 70 \%,-:>99)$ as yellow oil after chromatography on silica gel (100:1 hexane/EtOAc). ${ }^{1} \mathrm{H}$ NMR $\left(500 \mathrm{MHz}, \mathrm{CDCl}_{3}\right) \delta 7.67$ (dd, $J=2.9,1.2 \mathrm{~Hz}$, $1 \mathrm{H}), 7.58(\mathrm{dd}, J=5.0,1.2 \mathrm{~Hz}, 1 \mathrm{H}), 7.34(\mathrm{dd}, J=5.0,3.0 \mathrm{~Hz}, 1 \mathrm{H}), 7.13-7.09(\mathrm{~m}, 2 \mathrm{H}), 7.04$ $(\mathrm{d}, J=8.3 \mathrm{~Hz}, 2 \mathrm{H}), 6.31(\mathrm{~d}, J=7.3 \mathrm{~Hz}, 2 \mathrm{H}), 6.06(\mathrm{~s}, 1 \mathrm{H}), 5.76(\mathrm{~s}, 2 \mathrm{H}), 0.22(\mathrm{~s}, 9 \mathrm{H}) .{ }^{13} \mathrm{C}\{\mathrm{H}\}$ $\operatorname{NMR}\left(126 \mathrm{MHz}, \mathrm{CDCl}_{3}\right) \delta 140.8,140.1,136.5,128.7,127.7,125.5,125.0,120.1,118.2,114.3$, $106.3,104.3,103.1,-0.2$. The boron-bound carbon was not detected due to quadrupolar relaxation. HRMS (APCI) $m / z$ calcd for $\mathrm{C}_{21} \mathrm{H}_{21} \mathrm{BN}_{2} \mathrm{SSi}$, [M-H] $]^{+}$373.1366. Found: 373.1361 . 
<smiles>CC#CC=C(CCC)Cc1ccccc1</smiles>

The titled compound was isolated $(55.3 \mathrm{mg}, 76 \%, 4: 96)$ as colorless oil after chromatography on silica gel (100:1 hexane/EtOAc). ${ }^{1} \mathrm{H}$ NMR $\left(400 \mathrm{MHz}, \mathrm{CDCl}_{3}\right) \delta 6.21(\mathrm{~s}, 1 \mathrm{H}), 2.36(\mathrm{t}, J=$ $7.5 \mathrm{~Hz}, 2 \mathrm{H}), 1.50-1.41(\mathrm{~m}, 2 \mathrm{H}), 1.24(\mathrm{~s}, 12 \mathrm{H}), 0.90(\mathrm{t}, J=7.4 \mathrm{~Hz}, 3 \mathrm{H}), 0.19(\mathrm{~s}, 9 \mathrm{H}) .{ }^{13} \mathrm{C}\{\mathrm{H}\}$ $\operatorname{NMR}\left(126 \mathrm{MHz}, \mathrm{CDCl}_{3}\right) \delta 122.1,103.1,102.4,83.7,34.1,24.8,22.7,14.2,0.1$. The boronbound carbon was not detected due to quadrupolar relaxation. LRMS (EI) $\mathrm{m} / \mathrm{z}$ calcd for $\mathrm{C}_{16} \mathrm{H}_{29} \mathrm{BO}_{2} \mathrm{Si}$, 292.20. Found: 292.00.

(Z)-tert-butyldimethyl((3-(4,4,5,5-tetramethyl-1,3,2-dioxaborolan-2-yl)-6-(trimethylsilyl)hex-3-en-5-yn-1yl)oxy)silane (3o')

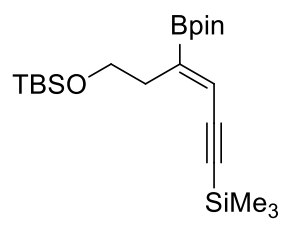

The titled compound was isolated $(58.2 \mathrm{mg}, 71 \%,-:>99)$ as yellow oil after chromatography on silica gel (100:1 hexane/EtOAc). ${ }^{1} \mathrm{H}$ NMR $\left(500 \mathrm{MHz}, \mathrm{CDCl}_{3}\right) \delta 6.27(\mathrm{~s}, 1 \mathrm{H}), 3.65(\mathrm{t}$, 2H), $2.64(\mathrm{t}, J=7.5 \mathrm{~Hz}, 2 \mathrm{H}), 1.23(\mathrm{~s}, 12 \mathrm{H}), 0.89(\mathrm{~s}, 9 \mathrm{H}), 0.18(\mathrm{~s}, 9 \mathrm{H}), 0.06(\mathrm{~s}, 6 \mathrm{H}) .{ }^{13} \mathrm{C}\{\mathrm{H}\}$ NMR $\left(126 \mathrm{MHz}, \mathrm{CDCl}_{3}\right) \delta 124.0,102.9,102.7,83.8,62.4,36.0,26.2,24.9,18.5,0.1,-5.0$. The boron-bound carbon was not detected due to quadrupolar relaxation. LR-MS (EI) $\mathrm{m} / \mathrm{z}$ calcd for $\mathrm{C}_{21} \mathrm{H}_{41} \mathrm{BO}_{3} \mathrm{Si}_{2}$, 408.27. Found: 408.00.

(Z)-(7-(benzyloxy)-4-(4,4,5,5-tetramethyl-1,3,2-dioxaborolan-2-yl)hept-3-en-1-yn-1-yl)trimethylsilane (3p')

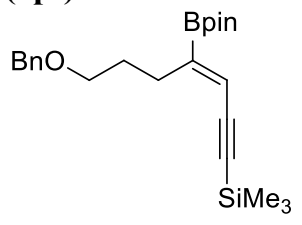

The titled compound was isolated $(61.2 \mathrm{mg}, 61 \%,-:>99)$ as yellow oil after chromatography on silica gel (50:1 hexane/EtOAc). ${ }^{1} \mathrm{H}$ NMR $\left(500 \mathrm{MHz}, \mathrm{CDCl}_{3}\right) \delta 7.36-$ $7.31(\mathrm{~m}, 5 \mathrm{H}), 6.22(\mathrm{~s}, 1 \mathrm{H}), 4.51(\mathrm{~s}, 2 \mathrm{H}), 3.48(\mathrm{t}, J=7.0 \mathrm{~Hz}, 2 \mathrm{H}), 2.45(\mathrm{t}, J=7.5 \mathrm{~Hz}, 2 \mathrm{H})$, $1.83-1.75(\mathrm{~m}, 2 \mathrm{H}), 1.23(\mathrm{~s}, 12 \mathrm{H}), 0.17(\mathrm{~s}, 9 \mathrm{H}) .{ }^{13} \mathrm{C}\{\mathrm{H}\} \mathrm{NMR}\left(126 \mathrm{MHz}, \mathrm{CDCl}_{3}\right) \delta 138.9$, $128.4,127.8,127.5,122.4,103.0,102.7,83.8,72.9,70.6,29.3,28.8,24.8,0.1,0.02$. The boron-bound carbon was not detected due to quadrupolar relaxation. LR-MS (EI) $m / z$ calcd for $\mathrm{C}_{23} \mathrm{H}_{35} \mathrm{BO}_{3} \mathrm{Si}_{2}$, 398.24. Found: 398.00 .

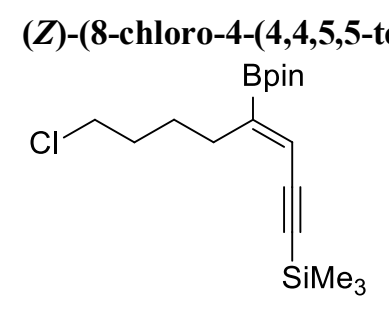

The titled compound was isolated $(54.4 \mathrm{mg}, 64 \%,-:>99)$ as yellow oil after chromatography on silica gel (100:1 hexane/EtOAc). ${ }^{1} \mathrm{H}$ NMR $\left(500 \mathrm{MHz}, \mathrm{CDCl}_{3}\right) \delta$ $6.23(\mathrm{~s}, 1 \mathrm{H}), 3.56(\mathrm{t}, J=6.8 \mathrm{~Hz}, 2 \mathrm{H}), 2.41(\mathrm{t}, J=7.3 \mathrm{~Hz}, 2 \mathrm{H}), 1.79-1.73(\mathrm{~m}, 2 \mathrm{H})$, $1.62-1.56(\mathrm{~m}, 2 \mathrm{H}), 1.24(\mathrm{~s}, 12 \mathrm{H}), 0.19(\mathrm{~s}, 9 \mathrm{H}) .{ }^{13} \mathrm{C}\{\mathrm{H}\} \mathrm{NMR}\left(126 \mathrm{MHz}, \mathrm{CDCl}_{3}\right) \delta$ $122.7,103.0,102.7,83.8,45.1,32.2,30.9,26.4,24.8,0.1$. The boron-bound carbon was not detected due to quadrupolar relaxation. LR-MS (EI) $m / z$ calcd for $\mathrm{C}_{17} \mathrm{H}_{30} \mathrm{BClO}_{2} \mathrm{Si}, 340.17$ Found: 340.00 .

\section{(Z)-2-(1,4-bis(4-methoxyphenyl)but-1-en-3-yn-1-yl)-2,3-dihydro-1H-naphtho[1,8-de][1,3,2]diazaborinine} (3r)<smiles>COc1ccc(C#C/C=C(/C(=O)c2ccccc2)c2ccc(OC)cc2)cc1</smiles>

The titled compound was isolated $(68.9 \mathrm{mg}, 64 \%,-:>99)$ as dark yellow oil after chromatography on silica gel (50:1 hexane/EtOAc). ${ }^{1} \mathrm{H}$ NMR $\left(400 \mathrm{MHz}, \mathrm{CDCl}_{3}\right) \delta 7.56(\mathrm{~d}$, $J=8.8 \mathrm{~Hz}, 2 \mathrm{H}), 7.29(\mathrm{~d}, J=8.8 \mathrm{~Hz}, 2 \mathrm{H}), 7.13-7.08(\mathrm{~m}, 2 \mathrm{H}), 7.04(\mathrm{~d}, J=7.6 \mathrm{~Hz}, 2 \mathrm{H})$, $6.98(\mathrm{~d}, J=8.8 \mathrm{~Hz}, 2 \mathrm{H}), 6.83(\mathrm{~d}, J=8.9 \mathrm{~Hz}, 2 \mathrm{H}), 6.33-6.28(\mathrm{~m}, 3 \mathrm{H}), 5.74(\mathrm{~s}, 2 \mathrm{H}), 3.88$ $(\mathrm{s}, 3 \mathrm{H}), 3.81(\mathrm{~s}, 3 \mathrm{H}) .{ }^{13} \mathrm{C}\{\mathrm{H}\} \mathrm{NMR}\left(101 \mathrm{MHz}, \mathrm{CDCl}_{3}\right) \delta 159.9,159.3,141.0,136.5,133.2$, 132.2, 130.7, 127.7, 120.1, 118.0, 115.6, 115.5, 114.2, 113.7, 106.2, 96.0, 87.8, 55.5, 55.4. The boron-bound carbon was not detected due to quadrupolar relaxation. HRMS (APCI) $m / z$ calcd for $\mathrm{C}_{28} \mathrm{H}_{23} \mathrm{BN}_{2} \mathrm{O}_{2},[\mathrm{M}-\mathrm{H}]^{+}$431.1931. Found: 431.1928 . 
(Z)-2-(1,4-bis(4-ethylphenyl)but-1-en-3-yn-1-yl)-2,3-dihydro-1H-naphtho[1,8-de][1,3,2]diazaborinine (3s)<smiles>CCc1ccc(C#C/C=C(/C(=O)c2ccccc2)c2ccc(CC)cc2)cc1</smiles>

The titled compound was isolated (78.4 mg, 74\%,-:>99) as yellow solid after chromatography on silica gel (50:1 hexane/EtOAc). ${ }^{1} \mathrm{H} \mathrm{NMR}\left(500 \mathrm{MHz}, \mathrm{CDCl}_{3}\right) \delta 7.54(\mathrm{~d}, J=8.1 \mathrm{~Hz}, 2 \mathrm{H})$, $7.31-7.27(\mathrm{~m}, 4 \mathrm{H}), 7.16-7.10(\mathrm{~m}, 4 \mathrm{H}), 7.06(\mathrm{~d}, J=8.2 \mathrm{~Hz}, 2 \mathrm{H}), 6.37(\mathrm{~s}, 1 \mathrm{H}), 6.31(\mathrm{~d}, J=$ $7.2 \mathrm{~Hz}, 2 \mathrm{H}), 5.77(\mathrm{~s}, 2 \mathrm{H}), 2.75(\mathrm{q}, J=7.6 \mathrm{~Hz}, 2 \mathrm{H}), 2.66(\mathrm{q}, J=7.7 \mathrm{~Hz}, 2 \mathrm{H}), 1.34(\mathrm{t}, J=7.6$ $\mathrm{Hz}, 3 \mathrm{H}), 1.25(\mathrm{t}, J=7.6 \mathrm{~Hz}, 3 \mathrm{H}) .{ }^{13} \mathrm{C}\{\mathrm{H}\} \mathrm{NMR}\left(126 \mathrm{MHz}, \mathrm{CDCl}_{3}\right) \delta 145.0,143.9,141.0$, 137.0, 136.4, 131.7, 129.3, 128.0, 127.8, 127.7, 120.7, 120.1, 118.0, 116.1, 106.2, 96.2, 88.3, $29.0,28.8,15.7,15.5$. The boron-bound carbon was not detected due to quadrupolar relaxation. HRMS (APCI) $m / z$ calcd for $\mathrm{C}_{30} \mathrm{H}_{27} \mathrm{BN}_{2}$, [M-H] $]^{+}$427.2346. Found: 427.2355.

\section{(Z)-2,2,3,3,16,16,17,17-octamethyl-8-(4,4,5,5-tetramethyl-1,3,2-dioxaborolan-2-yl)-4,15-dioxa-3,16- disilaoctadec-8-en-10-yne (3t')}

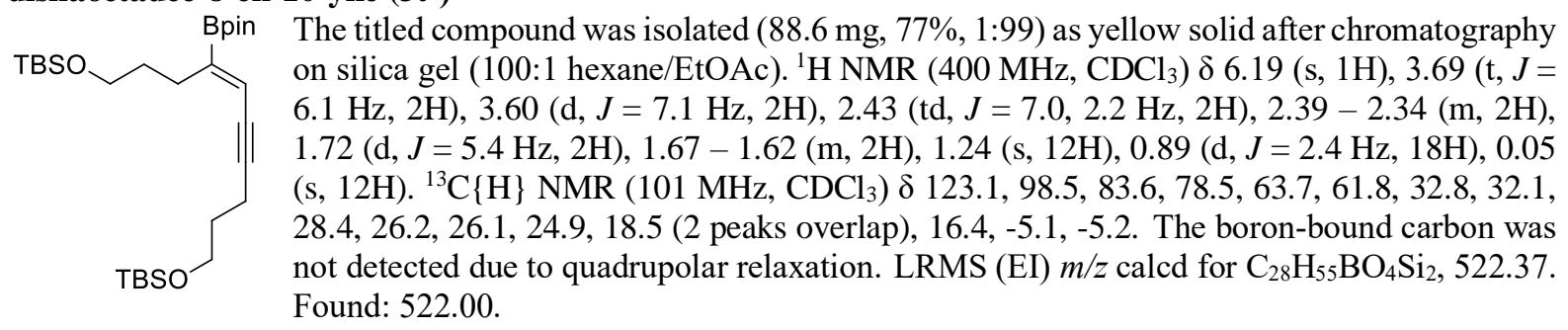

\section{(Z)-2-(1,10-dichlorodec-4-en-6-yn-4-yl)-4,4,5,5-tetramethyl-1,3,2-dioxaborolane (3u')}

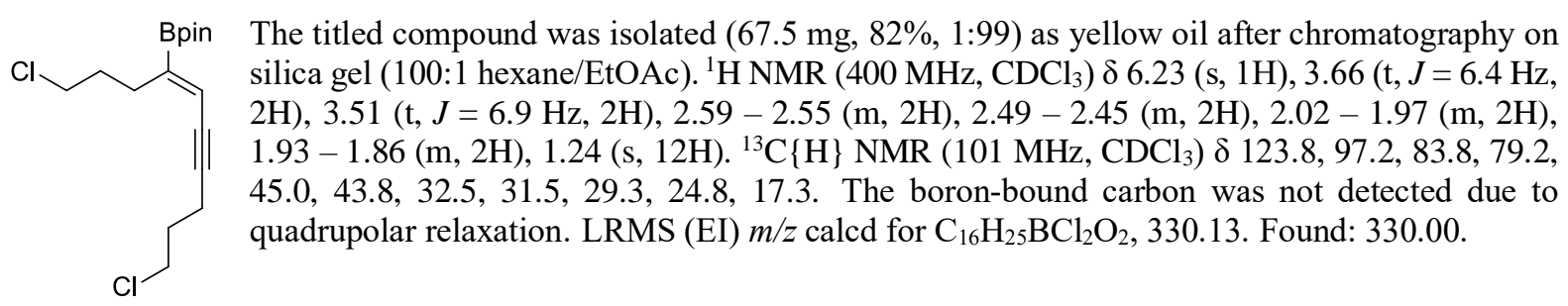

(Z)-4,4,5,5-tetramethyl-2-(1-phenyloct-3-en-1-yn-4-yl)-1,3,2-dioxaborolane (3v')<smiles>CCCCC#C/C(=C\c1ccccc1)c1ccccc1</smiles>

(90:10)
The titled compound was isolated $(59.0 \mathrm{mg}, 74 \%, 90: 10 *$ - refer to diagram) as yellow oil after chromatography on silica gel (100:1 hexane/EtOAc). ${ }^{1} \mathrm{H}$ NMR $\left(400 \mathrm{MHz}, \mathrm{CDCl}_{3}\right) \delta 7.46-7.43(\mathrm{~m}$, $2 \mathrm{H}), 7.33-7.30(\mathrm{~m}, 3 \mathrm{H}), 6.43(\mathrm{~s}, 1 \mathrm{H}), 2.50(\mathrm{~d}, J=7.4 \mathrm{~Hz}, 2 \mathrm{H})$, $1.50-1.43(\mathrm{~m}, 2 \mathrm{H}), 1.43-1.35(\mathrm{~m}, 2 \mathrm{H}), 1.28(\mathrm{~s}, 12 \mathrm{H}), 0.95(\mathrm{~d}, J$ $=7.3 \mathrm{~Hz}, 3 \mathrm{H}) .{ }^{13} \mathrm{C}\{\mathrm{H}\} \mathrm{NMR}\left(101 \mathrm{MHz}, \mathrm{CDCl}_{3}\right) \delta 131.7,128.4$, $128.3,123.8,121.9,97.0,87.6,83.7,31.9,31.8,24.8,22.7,14.2$. The boron-bound carbon was not detected due to quadrupolar relaxation. LR-MS (EI) $\mathrm{m} / z$ calcd for $\mathrm{C}_{20} \mathrm{H}_{27} \mathrm{BO}_{2}, 310.21$. Found:

310.00.

(Z)-2-(1,4-diphenylbut-1-en-3-yn-1-yl)-4,4,5,5-tetramethyl-1,3,2-dioxaborolane (4)<smiles></smiles>

The titled compound was isolated $(64.4 \mathrm{mg}, 78 \%, 2: 98)$ as brown oil after chromatography on silica gel (100:1 hexane/EtOAc). ${ }^{1} \mathrm{H} \mathrm{NMR}\left(400 \mathrm{MHz}, \mathrm{CDCl}_{3}\right) \delta 7.69(\mathrm{dd}, J=8.3,1.2 \mathrm{~Hz}, 2 \mathrm{H})$, $7.40-7.36(\mathrm{~m}, 3 \mathrm{H}), 7.35-7.33(\mathrm{~m}, 2 \mathrm{H}), 7.30-7.28(\mathrm{~m}, 3 \mathrm{H}), 6.72(\mathrm{~s}, 1 \mathrm{H}), 1.32(\mathrm{~s}, 12 \mathrm{H}) .{ }^{13} \mathrm{C}\{\mathrm{H}\}$ NMR $\left(101 \mathrm{MHz}, \mathrm{CDCl}_{3}\right) \delta 139.3,131.9,129.3,128.6,128.4,127.8,127.5,123.5,122.2,97.0$, $88.9,84.2,24.9$. The boron-bound carbon was not detected due to quadrupolar relaxation. LR-MS (EI) $\mathrm{m} / z$ calcd for $\mathrm{C}_{22} \mathrm{H}_{23} \mathrm{BO}_{2}, 330.17$. Found: 330.00 . 


\section{Determination of regio- and stereochemistry of 2e' and 3e' by NOE and $2 \mathrm{D}$ HMBC analysis:}

(Z)-(4-(4-methoxyphenyl)-4-(4,4,5,5-tetramethyl-1,3,2-dioxaborolan-2-yl)but-3-en-1-yn-1yl)trimethylsilane (2e')<smiles>CC#C/C(=C\c1ccc(OC)cc1)Cc1ccccc1</smiles>

The titled compound was isolated $(62.0 \mathrm{mg}, 70 \%)$ as pale yellow oil after chromatography on silica gel (100:1 hexane/EtOAc). ${ }^{1} \mathrm{H}$ NMR $\left(400 \mathrm{MHz}, \mathrm{CDCl}_{3}\right) \delta 8.06$ $(\mathrm{d}, J=8.8 \mathrm{~Hz}, 2 \mathrm{H}), 7.34(\mathrm{~s}, 1 \mathrm{H}), 6.87(\mathrm{~d}, J=8.9 \mathrm{~Hz}, 2 \mathrm{H}), 3.84(\mathrm{~s}, 3 \mathrm{H}), 1.31(\mathrm{~s}, 12 \mathrm{H})$, $0.26(\mathrm{~s}, 9 \mathrm{H}) .{ }^{13} \mathrm{C}\{\mathrm{H}\} \mathrm{NMR}\left(126 \mathrm{MHz}, \mathrm{CDCl}_{3}\right) \delta 160.6,150.8,131.7,130.3,113.5,106.3$, $102.4,84.2,55.4,24.9,0.2$. The boron-bound carbon was not detected due to quadrupolar relaxation. GC-MS (EI) $m / z$ : calcd for $\mathrm{C}_{20} \mathrm{H}_{29} \mathrm{BO}_{3} \mathrm{Si}[\mathrm{M}]^{+}: 356.19$; Found: 356.00

(Z)-(4-(4-methoxyphenyl)-4-(4,4,5,5-tetramethyl-1,3,2-dioxaborolan-2-yl)but-3-en-1-yn-1yl)trimethylsilane (3e')<smiles>CC#C/C=C(/c1ccccc1)c1ccc(OC)cc1</smiles>

The titled compound was isolated $(55.0 \mathrm{mg}, 62 \%)$ as colorless oil after chromatography on silica gel (100:1 hexane/EtOAc). ${ }^{1} \mathrm{H}$ NMR $\left(400 \mathrm{MHz}, \mathrm{CDCl}_{3}\right) \delta 7.68(\mathrm{~d}, J=8.9 \mathrm{~Hz}$, $2 \mathrm{H}), 6.86(\mathrm{~d}, J=8.9 \mathrm{~Hz}, 2 \mathrm{H}), 6.41(\mathrm{~s}, 1 \mathrm{H}), 3.82(\mathrm{~s}, 3 \mathrm{H}), 1.29(\mathrm{~s}, 12 \mathrm{H}), 0.15(\mathrm{~s}, 9 \mathrm{H}) .{ }^{13} \mathrm{C}\{\mathrm{H}\}$ NMR $\left(126 \mathrm{MHz}, \mathrm{CDCl}_{3}\right) \delta 159.1,131.5,130.6,120.1,113.1,104.4,103.1,84.1,55.4$, $24.9,-0.2$. The boron-bound carbon was not detected due to quadrupolar relaxation. GCMS (EI) $m / z$ : calcd for $\mathrm{C}_{20} \mathrm{H}_{29} \mathrm{BO}_{3} \mathrm{Si}[\mathrm{M}]^{+}: 356.19$; Found: 356.00

1D NOE<smiles>C#C/C(Br)=C\c1ccc(OC)cc1</smiles>

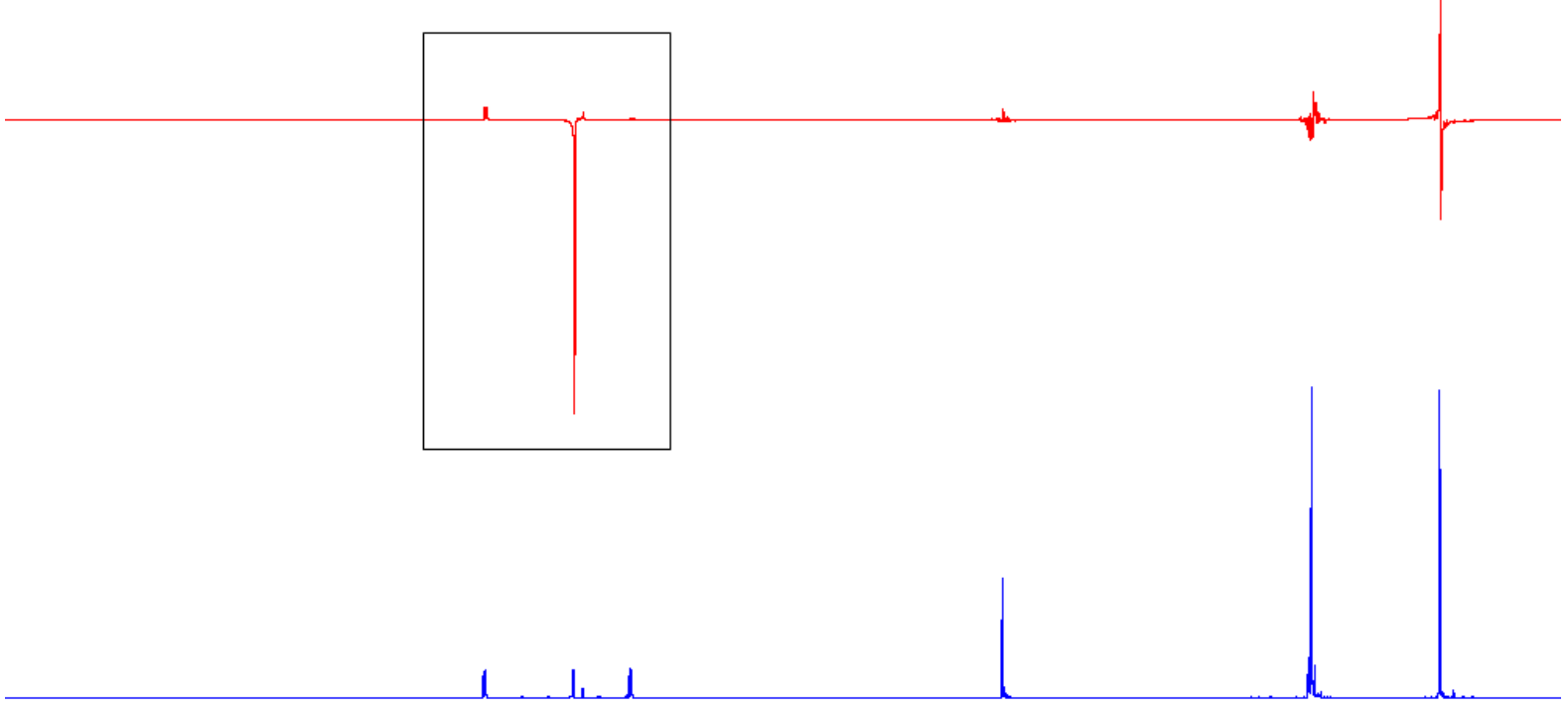




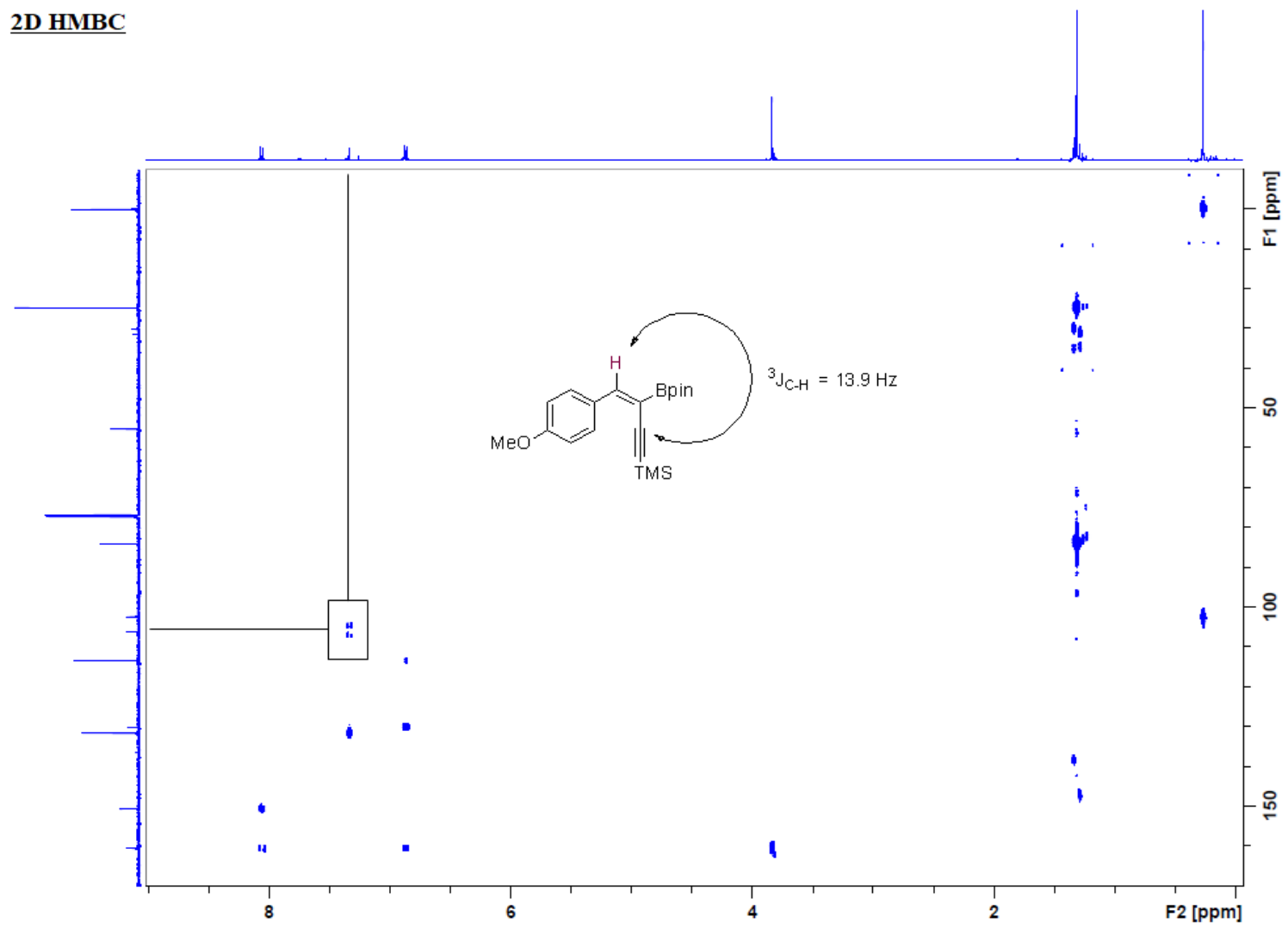



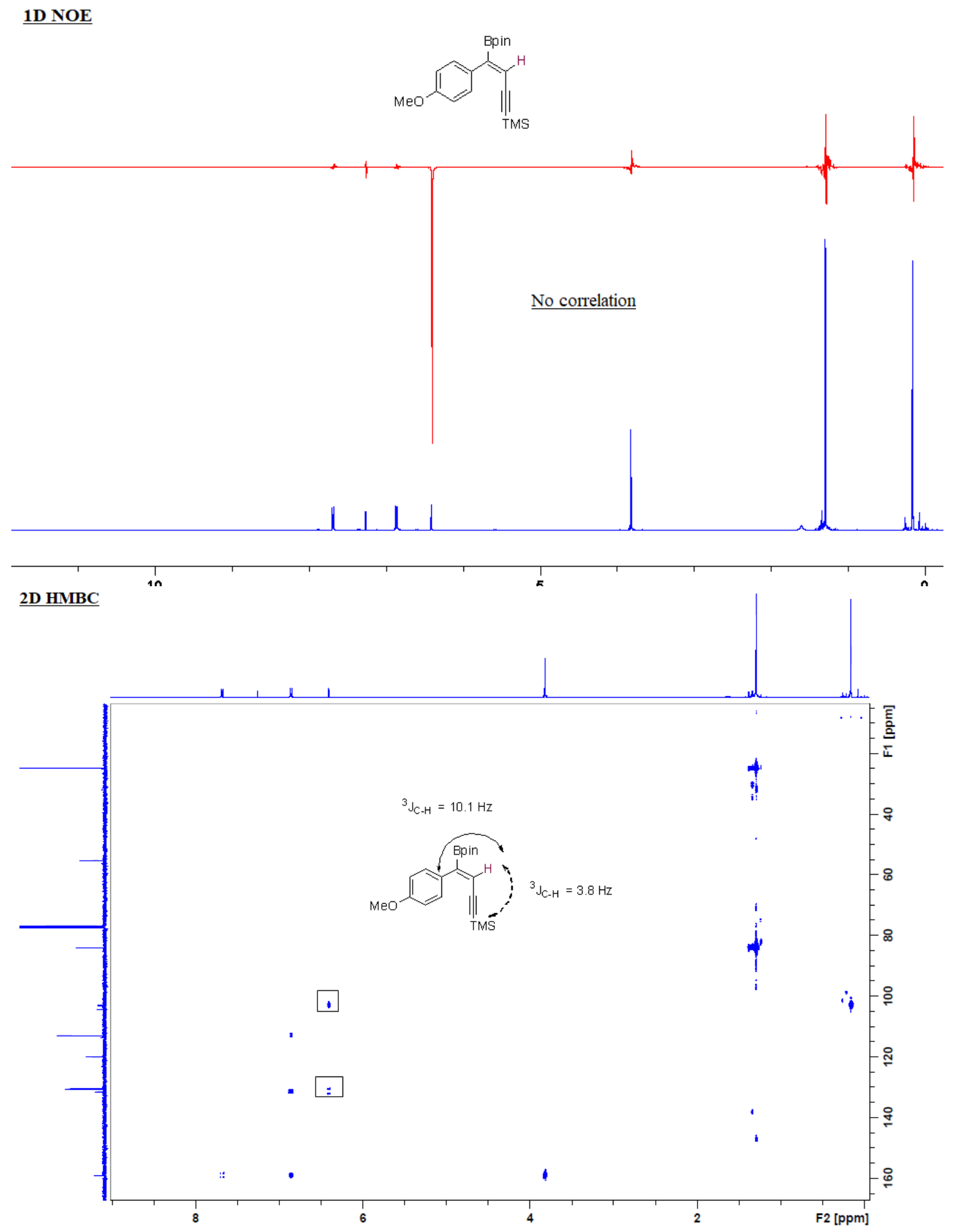


\section{General procedure for gram scale for Co-catalyzed $\beta$-hydroboration of 1,3-diynes}

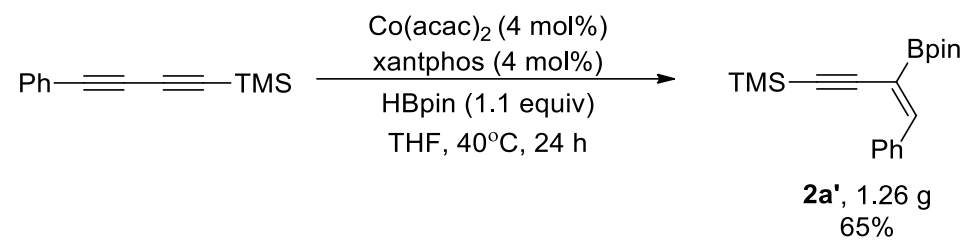

$\mathrm{Co}(\mathrm{acac})_{2}(51.6 \mathrm{mg}, 0.200 \mathrm{mmol})$ and xantphos $(115.7 \mathrm{mg}, 0.200 \mathrm{mmol})$ were weighted and added into a $100 \mathrm{~mL}$ Schlenk flask containing $4 \mathrm{~mL}$ of THF inside the glovebox. The resulting mixture was stirred for 15 mins before adding HBpin (0.70 g, $5.5 \mathrm{mmol})$ and trimethyl(phenylbuta-1,3-diyn-1-yl)silane (1.00 g, $5.0 \mathrm{mmol})$ successively. The Schlenk flask was removed from the glove box, and the mixture was stirred at $40{ }^{\circ} \mathrm{C}$ for 24 hours. The residue was then purified by flash column chromatography using a mixture of hexane and ethyl acetate (100:1) as eluent yielding (Z)-trimethyl(4-phenyl-3-(4,4,5,5-tetramethyl-1,3,2-dioxaborolan-2-yl)but-3-en-1-yn-1-yl)silane,2a' $(1.26 \mathrm{~g}, 65 \%,>99:-)$ as a dark brown solid.

(Z)-trimethyl(4-phenyl-3-(4,4,5,5-tetramethyl-1,3,2-dioxaborolan-2-yl)but-3-en-1-yn-1-yl)silane (2a')

${ }^{1} \mathrm{H}$ NMR $\left(500 \mathrm{MHz}, \mathrm{CDCl}_{3}\right) \delta 8.06(\mathrm{~d}, J=6.6 \mathrm{~Hz}, 2 \mathrm{H}), 7.39(\mathrm{~s}, 1 \mathrm{H}), 7.38-7.32(\mathrm{~m}, 3 \mathrm{H}), 1.32(\mathrm{~s}, 12 \mathrm{H}), 0.26(\mathrm{~s}$, $9 \mathrm{H}) .{ }^{13} \mathrm{C}\left\{{ }^{1} \mathrm{H}\right\} \mathrm{NMR}\left(126 \mathrm{MHz}, \mathrm{CDCl}_{3}\right) \delta 151.1,137.1,129.9,129.5,128.2,105.7,103.4,84.3,24.9,0.1$. The boron-bound carbon was not detected due to quadrupolar relaxation. GC-MS (EI) $m / z$ : calcd for $\mathrm{C}_{19} \mathrm{H}_{27} \mathrm{BO}_{2} \mathrm{Si}$ $[\mathrm{M}]^{+}:$326.19; Found: 326.0.

\section{General procedure for gram scale for Co-catalyzed $\alpha$-hydroboration of 1,3-diynes}

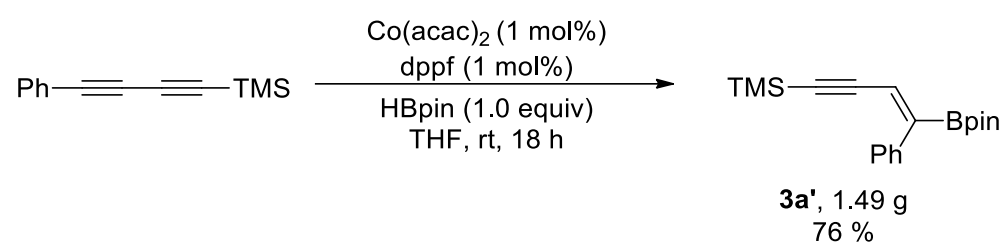

$\mathrm{Co}(\mathrm{acac})_{2}(12.9 \mathrm{mg}, 0.050 \mathrm{mmol})$ and $\mathrm{dppf}(27.7 \mathrm{mg}, 0.050 \mathrm{mmol})$ were weighted and added into a $100 \mathrm{~mL}$ Schlenk flask containing $20 \mathrm{~mL}$ of THF inside the glovebox. The resulting mixture was stirred for 15 mins before adding HBpin (0.64 g, $5.0 \mathrm{mmol})$ and trimethyl(phenylbuta-1,3-diyn-1-yl)silane (1.00 g, $5.0 \mathrm{mmol})$ successively. The Schlenk flask was removed from the glove box, and the mixture was stirred at room temperature for 18 hours. The residue was then purified by flash column chromatography using a mixture of hexane and ethyl acetate (100:1) as eluent yielding (Z)-trimethyl(4-phenyl-4-(4,4,5,5-tetramethyl-1,3,2-dioxaborolan-2-yl)but-3-en-1-yn-1yl)silane , 3a' $(1.49 \mathrm{~g}, 76 \%, 1: 99)$ as a dark brown solid.

\section{(Z)-trimethyl(4-phenyl-4-(4,4,5,5-tetramethyl-1,3,2-dioxaborolan-2-yl)but-3-en-1-yn-1-yl)silane (3a')}

${ }^{1} \mathrm{H}$ NMR $\left(500 \mathrm{MHz}, \mathrm{CDCl}_{3}\right) \delta 7.64(\mathrm{~d}, J=7.2 \mathrm{~Hz}, 2 \mathrm{H}), 7.32(\mathrm{t}, J=7.5 \mathrm{~Hz}, 3 \mathrm{H}), 6.48(\mathrm{~s}, 1 \mathrm{H}), 1.29(\mathrm{~s}, 12 \mathrm{H}), 0.13$ $(\mathrm{s}, 9 \mathrm{H}) .{ }^{13} \mathrm{C}\{\mathrm{H}\} \mathrm{NMR}\left(126 \mathrm{MHz}, \mathrm{CDCl}_{3}\right) \delta 138.9,129.2,127.6,127.5,121.9,104.0,103.4,84.2,24.9,-0.2$. The boron-bound carbon was not detected due to quadrupolar relaxation. GC-MS (EI) $m / z$ : calcd for $\mathrm{C}_{19} \mathrm{H}_{27} \mathrm{BO}_{2} \mathrm{Si}$ $[\mathrm{M}]^{+}:$326.19; Found: 326.0. 


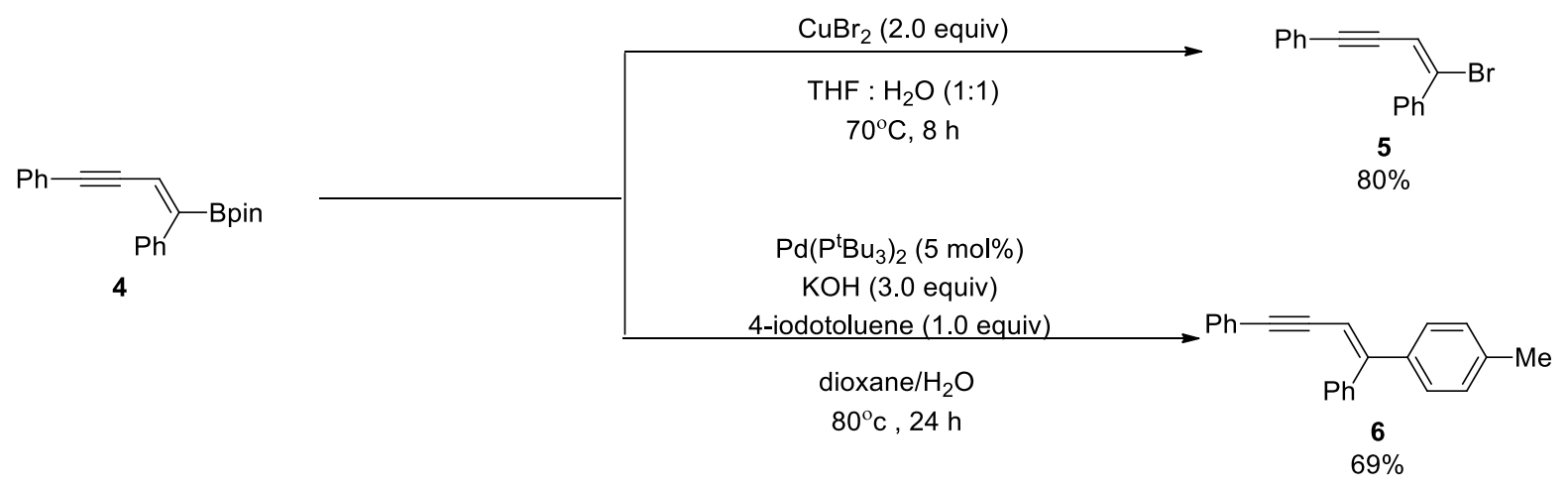

General procedure for Bromination of (Z)-2-(1,4-diphenylbut-1-en-3-yn-1-yl)-4,4,5,5-tetramethyl-1,3,2dioxaborolane $^{4}$

A mixture of (Z)-2-(1,4-diphenylbut-1-en-3-yn-1-yl)-4,4,5,5-tetramethyl-1,3,2-dioxaborolane, 4 (33.0 mg, 0.100 $\mathrm{mmol}), \mathrm{CuBr}_{2}(45.0 \mathrm{mg}, 0.200 \mathrm{mmol})$ was added into $0.4 \mathrm{~mL}$ of THF/ $\mathrm{H}_{2} \mathrm{O}(1: 1)$ mixture in a 4-mL screw-capped vial containing a magnetic stirring bar. Subsequently, it was stirred at $70{ }^{\circ} \mathrm{C}$ for 8 hours. After the completion of the reaction, the residue was extracted with ethyl acetate thrice, and the organic layer was dried over sodium sulfate prior concentrating it. It was then purified by flash column chromatography using 100:1 hexane/ethyl acetate as eluents yielding $(E)$-(1-bromobut-1-en-3-yne-1,4-diyl)dibenzene $(23.0 \mathrm{mg}, 80 \%)$ as a yellow oil.

\section{(E)-(1-bromobut-1-en-3-yne-1,4-diyl)dibenzene (5)}

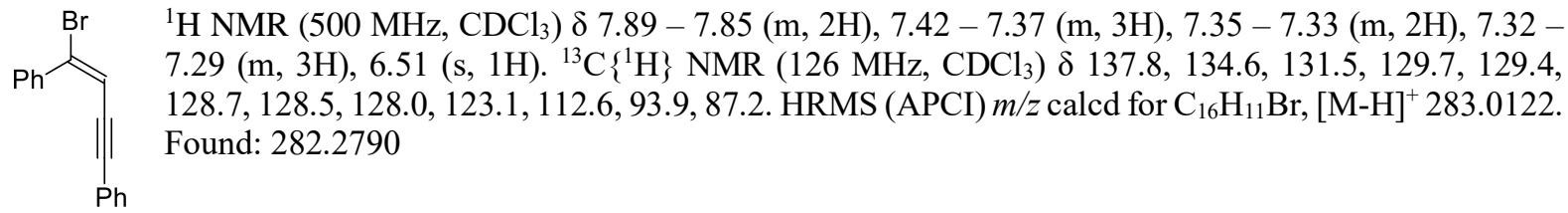

\section{General procedure for Suzuki cross-coupling of (Z)-2-(1,4-diphenylbut-1-en-3-yn-1-yl)-4,4,5,5- tetramethyl-1,3,2-dioxaborolane ${ }^{5}$}

A mixture of $\mathrm{Pd}\left(\mathrm{P}^{\mathrm{t}} \mathrm{Bu}_{3}\right)_{2}(2.5 \mathrm{mg}, 5.0 \mu \mathrm{mol})$, 4-iodotoluene $(21.8 \mathrm{mg}, 0.1 \mathrm{mmol}),(Z)-2$-(1,4-diphenylbut-1-en-3yn-1-yl)-4,4,5,5-tetramethyl-1,3,2-dioxaborolane, $4(33.0 \mathrm{mg}, 0.1 \mathrm{mmol})$ and potassium hydroxide $(20 \mathrm{mg}, 0.30$ mmol) was added into $1.5 \mathrm{~mL}$ of dioxane and $0.5 \mathrm{~mL}$ of water in $25 \mathrm{~mL}$ Schleck tube under $\mathrm{N}_{2}$ protection.. After stirring at $80{ }^{\circ} \mathrm{c}$ for 24 hours, the mixture was concentrated and purified by flash column chromatography using hexane and ethyl acetate (100:1) as eluents yielding (E)-(1-(p-tolyl)but-1-en-3-yne-1,4-diyl)dibenzene, $(20.2 \mathrm{mg}$, $69 \%$ ) as a dark red oil.<smiles>Cc1ccc(C(=CC#Cc2ccccc2)c2ccccc2)cc1</smiles>

\section{(E)-(1-(p-tolyl)but-1-en-3-yne-1,4-diyl)dibenzene (6)}

${ }^{1} \mathrm{H}$ NMR (500 MHz, $\left.\mathrm{CDCl}_{3}\right) \delta 7.63(\mathrm{~d}, J=7.0 \mathrm{~Hz}, 2 \mathrm{H}), 7.43-7.39(\mathrm{~m}, 2 \mathrm{H}), 7.36-7.30(\mathrm{~m}, 6 \mathrm{H}), 7.29$ $-7.25(\mathrm{~m}, 3 \mathrm{H}), 7.18(\mathrm{~d}, J=6.8 \mathrm{~Hz}, 1 \mathrm{H}), 5.96(\mathrm{~s}, 1 \mathrm{H}), 2.04(\mathrm{~s}, 3 \mathrm{H}) .{ }^{13} \mathrm{C}\left\{{ }^{1} \mathrm{H}\right\} \mathrm{NMR}\left(126 \mathrm{MHz}, \mathrm{CDCl}_{3}\right)$ $\delta$ 152.5, 142.2, 139.3, 136.6, 131.6, 130.5, 130.1, 129.2, 128.5, 128.3, 128.0, 125.9, 123.8, 108.8, 93.8, 88.9, 20.5.HRMS (APCI) $\mathrm{m} / z$ calcd for $\mathrm{C}_{23} \mathrm{H}_{18}[\mathrm{M}+\mathrm{H}]^{+}: 295.1487$ Found: 295.1480 

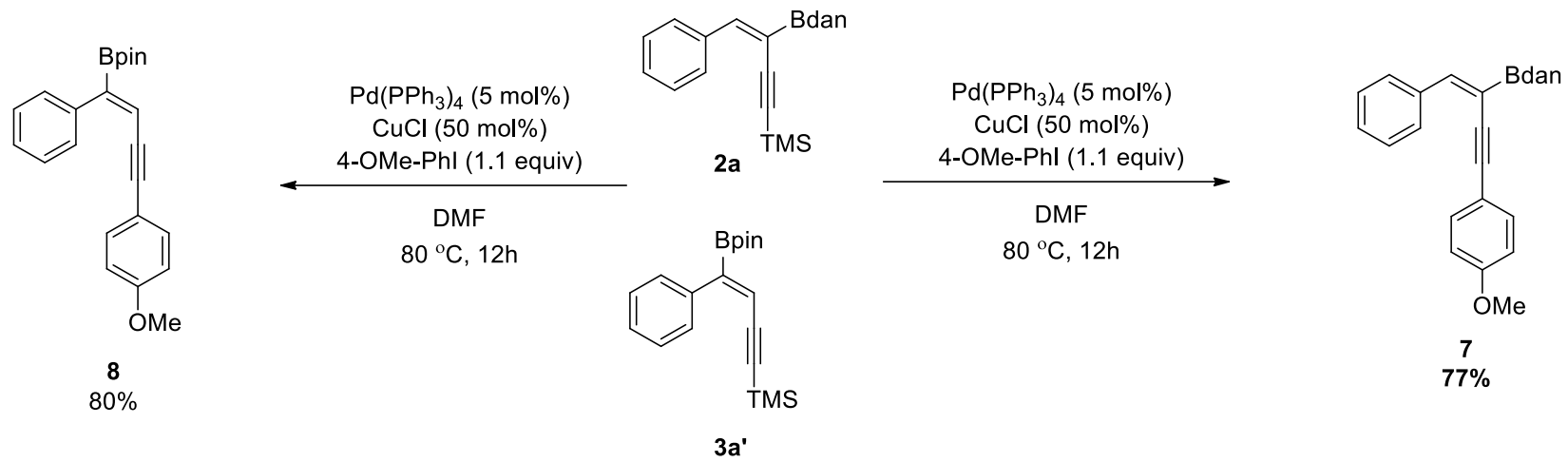

General procedure for Sila-Sonogashira cross-coupling ${ }^{6}$

A mixture of $\mathrm{Pd}\left(\mathrm{PPh}_{3}\right)_{4}(17.3 \mathrm{mg}, 15.0 \mu \mathrm{mol}), \mathrm{CuCl}(14.9 \mathrm{mg}, 0.15 \mathrm{mmol}), \mathbf{2} \mathbf{a}^{\prime} / 3 \mathbf{a}^{\prime}(99.0 \mathrm{mg}, 0.3 \mathrm{mmol})$ and $4-$ iodoanisole ( $77 \mathrm{mg}, 0.33 \mathrm{mmol}$ ) was added into $2 \mathrm{~mL}$ DMF in a $4 \mathrm{~mL}$ vial inside the glovebox. After stirring at $80{ }^{\circ} \mathrm{C}$ for 12 hours, the crude mixture was washed with saturated $\mathrm{NaCl}$ and extracted thrice with ethyl acetate. The organic layer was then dried over sodium sulfate, filtered and concentrated under vacuum. It was then purified by flash column chromatography to get the pure desired product.

\section{(Z)-2-(4-(4-methoxyphenyl)-1-phenylbut-1-en-3-yn-1-yl)-4,4,5,5-tetramethyl-1,3,2-dioxaborolane (7)}

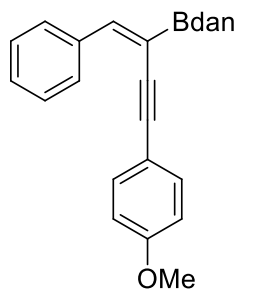

The reaction was conducted on a $0.2 \mathrm{mmol}$ scale. The titled compound was isolated ( $62.0 \mathrm{mg}$, $77 \%)$ as yellow powder after chromatography on silica gel $\left(50: 1\right.$ hexane/EtOAc). ${ }^{1} \mathrm{H} \mathrm{NMR}$ $\left(500 \mathrm{MHz}, \mathrm{CDCl}_{3}\right) \delta 8.08(\mathrm{~d}, J=7.4 \mathrm{~Hz}, 2 \mathrm{H}), 7.50(\mathrm{~d}, J=8.8 \mathrm{~Hz}, 2 \mathrm{H}), 7.46-7.41(\mathrm{~m}, 2 \mathrm{H})$, $7.36(\mathrm{t}, J=7.3 \mathrm{~Hz}, 1 \mathrm{H}), 7.18-7.12(\mathrm{~m}, 2 \mathrm{H}), 7.10(\mathrm{~s}, 1 \mathrm{H}), 7.06(\mathrm{~d}, J=8.2 \mathrm{~Hz}, 2 \mathrm{H}), 6.94(\mathrm{~d}, J$ $=8.8 \mathrm{~Hz}, 2 \mathrm{H}), 6.43(\mathrm{~d}, J=7.2 \mathrm{~Hz}, 2 \mathrm{H}), 6.12(\mathrm{~s}, 2 \mathrm{H}), 3.86(\mathrm{~s}, 3 \mathrm{H}) \cdot{ }^{13} \mathrm{C} \mathrm{NMR}\left(126 \mathrm{MHz}, \mathrm{CDCl}_{3}\right)$ $\delta 160 ., 143.1,141.1,137.2,136.5,133.1,129.5,129.2,128.5,127.7,120.1,118.0,115.9$, 114.4, 106.3, 99.0, 88.6, 55.5. The boron-bound carbon was not detected due to quadrupolar relaxation. HRMS (EI) $\mathrm{m} / z$ calcd for $\mathrm{C}_{27} \mathrm{H}_{22} \mathrm{BN}_{2} \mathrm{O}$ : 400.1747 Found: 400.1741

(Z)-2-(4-(4-methoxyphenyl)-1-phenylbut-1-en-3-yn-1-yl)-4,4,5,5-tetramethyl-1,3,2-dioxaborolane (8)<smiles>COc1ccc(C#C/C=C(\Cc2ccccc2)c2ccccc2)cc1</smiles>

The titled compound was isolated $(86.0 \mathrm{mg}, 80 \%)$ as brown oil after chromatography on silica gel (100:1 hexane/EtOAc). ${ }^{1} \mathrm{H}$ NMR $\left(500 \mathrm{MHz}, \mathrm{CDCl}_{3}\right) \delta 7.68(\mathrm{dd}, J=8.2,1.2 \mathrm{~Hz}, 2 \mathrm{H}), 7.37(\mathrm{t}$, $J=7.6 \mathrm{~Hz}, 2 \mathrm{H}), 7.28(\mathrm{~d}, J=8.9 \mathrm{~Hz}, 3 \mathrm{H}), 6.81(\mathrm{~d}, J=8.9 \mathrm{~Hz}, 2 \mathrm{H}), 6.70(\mathrm{~s}, 1 \mathrm{H}), 3.80(\mathrm{~s}, 3 \mathrm{H}), 1.32$ $(\mathrm{s}, 12 \mathrm{H}) .{ }^{13} \mathrm{C}\{\mathrm{H}\} \mathrm{NMR}\left(126 \mathrm{MHz}, \mathrm{CDCl}_{3}\right) \delta 156.0,139.5,133.4,129.3,127.7,127.3,122.6$, $115.7,114.1,97.3,88.0,84.1,55.4,24.9$. The boron-bound carbon was not detected due to quadrupolar relaxation. LRMS (EI) $\mathrm{m} / z$ calcd for $\mathrm{C}_{23} \mathrm{H}_{25} \mathrm{BO}_{3}[\mathrm{M}]^{+}: 360.19$ Found: 360.00 


\section{Deuterium studies}

Note: DBpin was synthesized based on previously reported procedure ${ }^{7}$.

For $\beta$-hydroboration of ((4-methoxyphenyl)buta-1,3-diyn-1-yl)trimethylsilane:

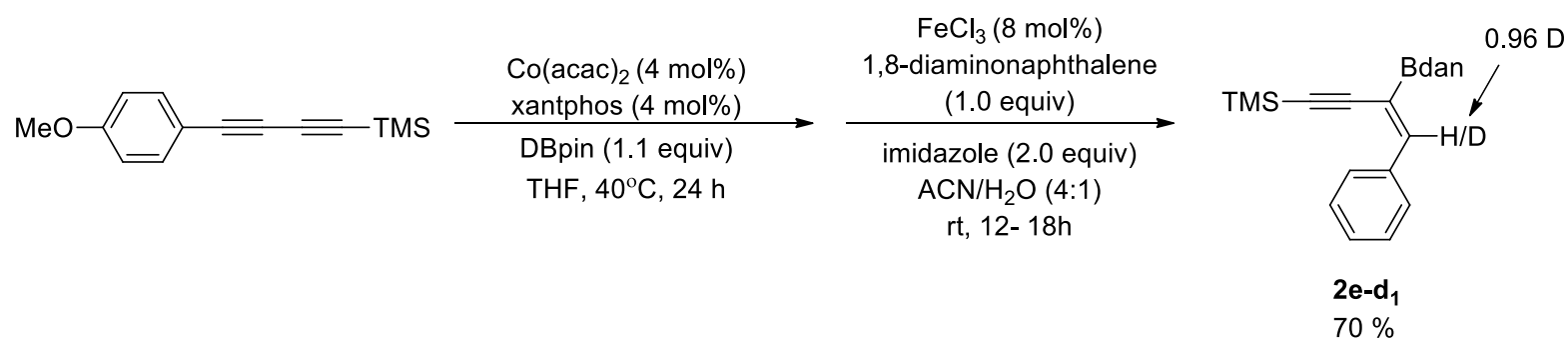

In an Ar-filled glovebox, a mixture of $\mathrm{Co}(\mathrm{acac})_{2}(2.4 \mathrm{mg}, 10.0 \mu \mathrm{mol})$ and xantphos $(5.8 \mathrm{mg}, 10.0 \mu \mathrm{mol})$ in THF $(0.2 \mathrm{~mL})$ was added into a $4-\mathrm{mL}$ screw-capped vial containing a magnetic stirring bar. The resulting mixture was stirred for 2 minutes before adding DBpin $(36.1 \mathrm{mg}, 0.280 \mathrm{mmol})$ and 1,3-diynes $(0.250 \mathrm{mmol})$ successively. The reaction mixture was stirred at $40{ }^{\circ} \mathrm{C}$ for 24 hours.

Upon reaction completion, $\mathrm{FeCl}_{3}(3.2 \mathrm{mg}, 20.0 \mu \mathrm{mol})$, 1,8-diaminonaphthalene ( $40.0 \mathrm{mg}, 0.250 \mathrm{mmol}$ ), imidazole $(34.0 \mathrm{mg}, 0.500 \mathrm{mmol})$ was added into another $4-\mathrm{mL}$ screw-capped vial containing a magnetic stirring bar containing water $(0.5 \mathrm{~mL})$ and acetonitrile $(0.5 \mathrm{~mL})$. Subsequently, the crude hydroboration reaction mixture was transferred and rinsed with acetonitrile $(1.5 \mathrm{~mL})$ into the latter vial. It was then stirred for 12 to $18 \mathrm{hr}$ at room temperature for the conversion to Bdan derivatives to take place. Lastly, the residue was purified by flash chromatography on silica using a mixture of ethyl acetate and hexane as eluent. Equimolar of chloroform- $d$ was added as the internal standard for ${ }^{2} \mathrm{H}$ NMR analysis.

For $\alpha$-hydroboration of ((4-methoxyphenyl)buta-1,3-diyn-1-yl)trimethylsilane:

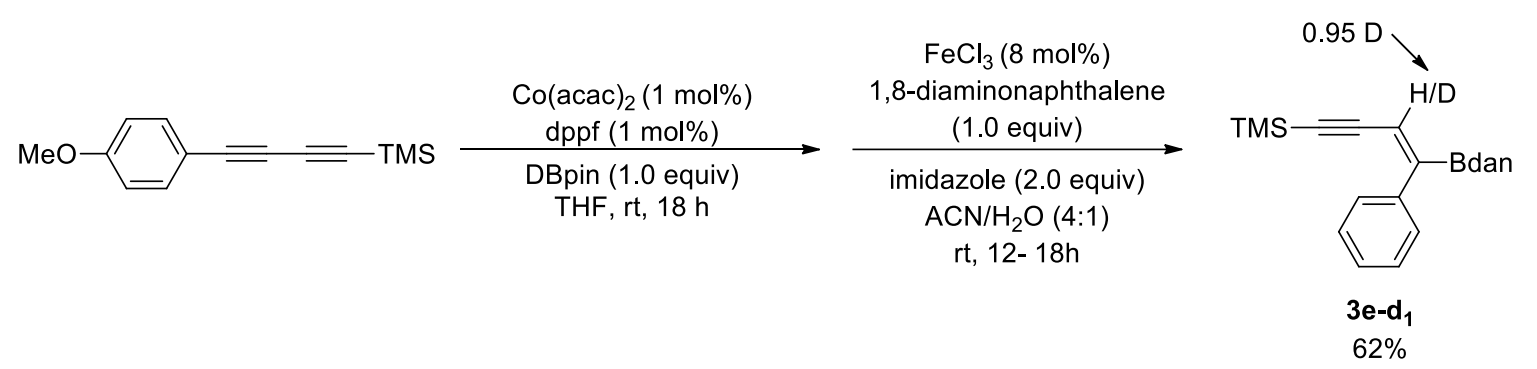

In an Ar-filled glovebox, a mixture of Co(acac $)_{2}(0.6 \mathrm{mg}, 2.5 \mu \mathrm{mol})$ and dppf $(1.4 \mathrm{mg}, 2.5 \mu \mathrm{mol})$ in THF $(0.5 \mathrm{~mL})$ was added into a 4-mL screw-capped vial containing a magnetic stirring bar. The resulting mixture was stirred for 2 minutes before adding DBpin $(32.3 \mathrm{mg}, 0.250 \mathrm{mmol})$ and 1,3-diynes $(0.250 \mathrm{mmol})$ successively. The reaction mixture was stirred at room temperature for 18 hours.

Upon reaction completion, $\mathrm{FeCl}_{3}(3.2 \mathrm{mg}, 20.0 \mu \mathrm{mol})$, 1,8-diaminonaphthalene (40.0 mg, $0.250 \mathrm{mmol}$ ), imidazole $(34.0 \mathrm{mg}, 0.500 \mathrm{mmol})$ was added into another $4-\mathrm{mL}$ screw-capped vial containing a magnetic stirring bar containing water $(0.5 \mathrm{~mL})$ and acetonitrile $(0.5 \mathrm{~mL})$. Subsequently, the crude hydroboration reaction mixture was transferred and rinsed with acetonitrile $(1.5 \mathrm{~mL})$ into the latter vial. It was then stirred for 12 to $18 \mathrm{hr}$ at room temperature for the conversion to Bdan derivatives to take place. Lastly, the residue was purified by flash chromatography on silica using a mixture of ethyl acetate and hexane as eluent. Chloroform- $d$ was added (2:1 molar ratio with respect to the product) as the internal standard for ${ }^{2} \mathrm{H}$ NMR analysis. 


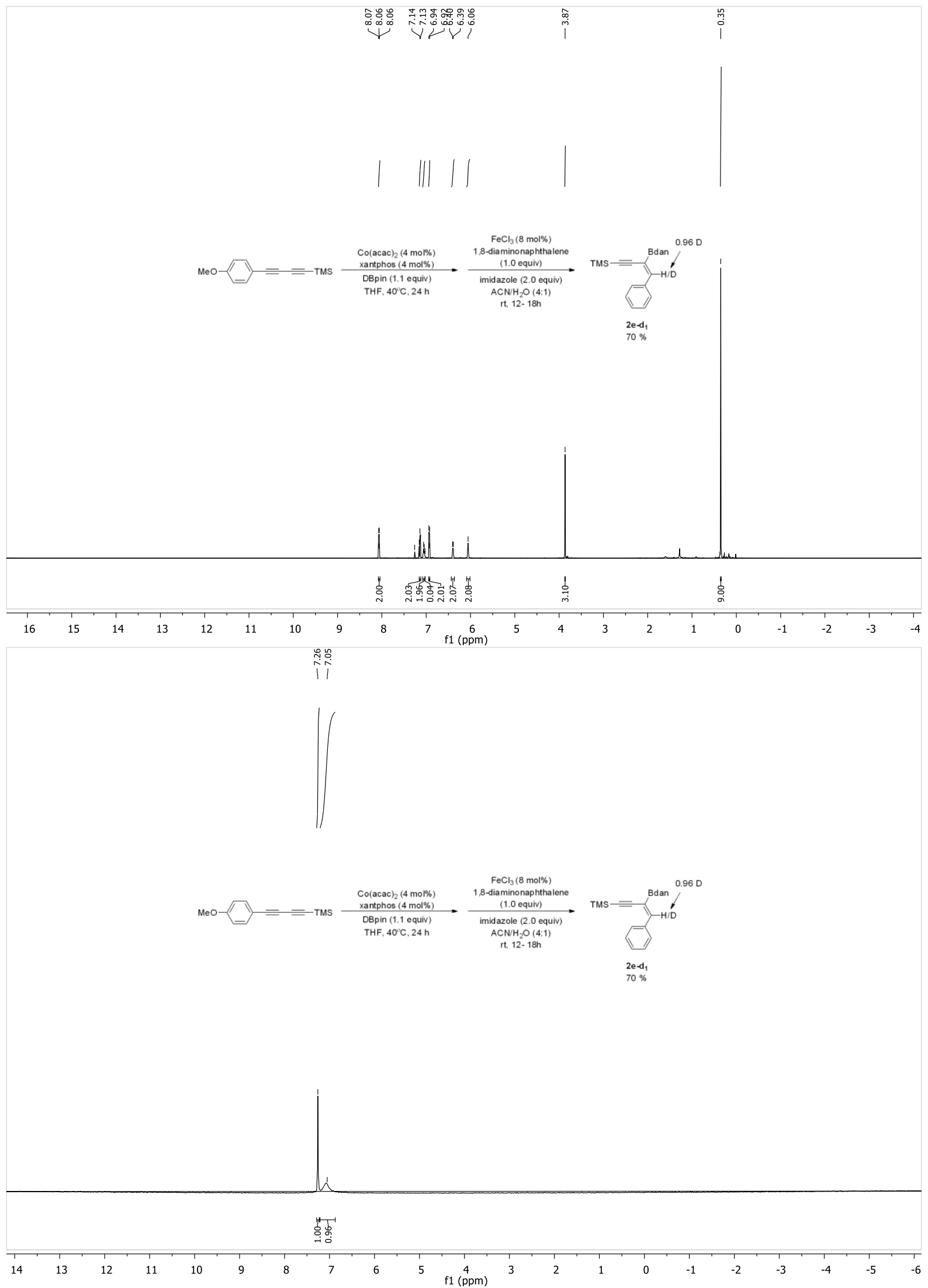




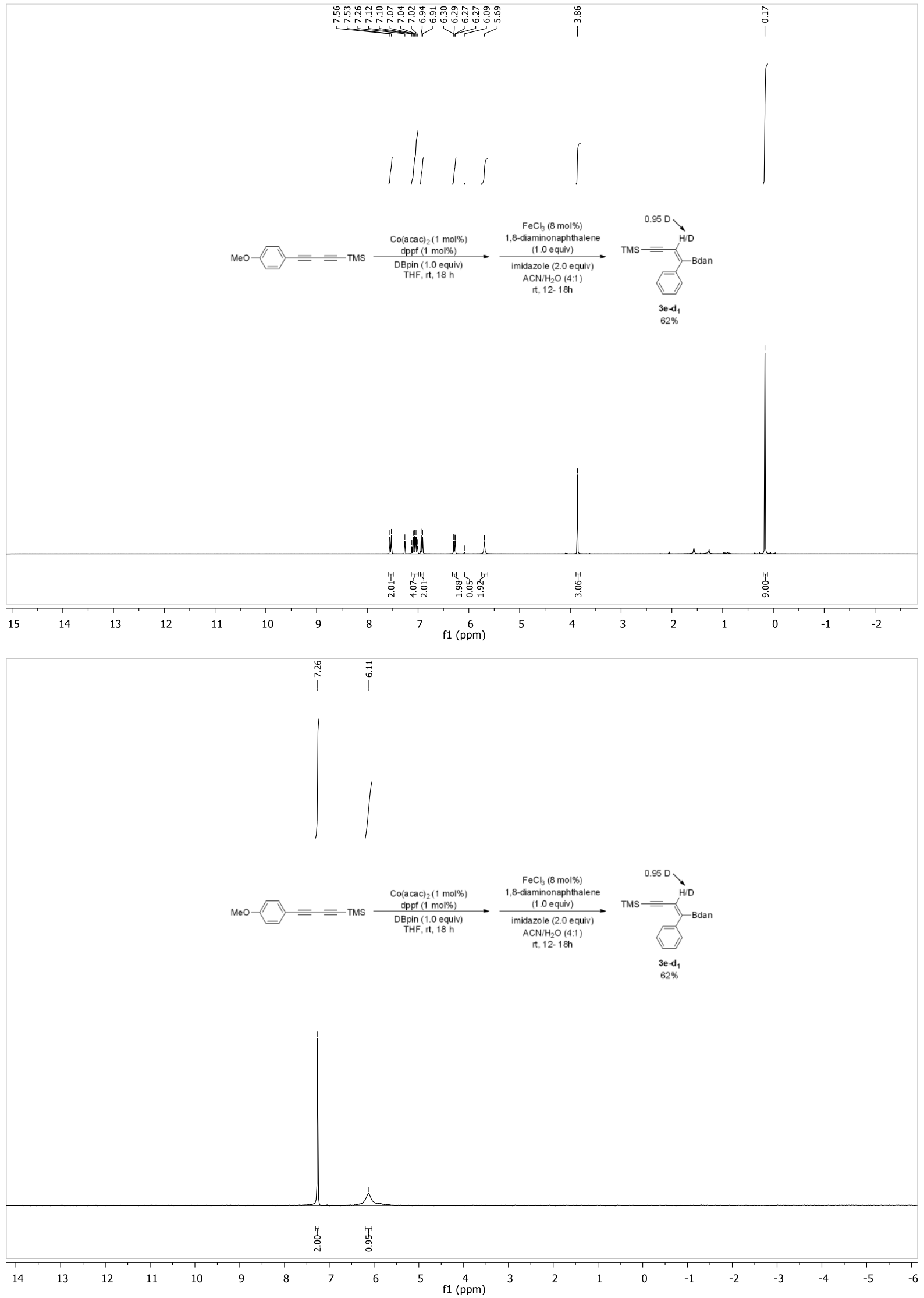




\section{References}

1. Balaraman, K.; Kesavan, V., Efficient Copper(II) Acetate Catalyzed Homo- and Heterocoupling of Terminal Alkynes at Ambient Conditions. Synthesis 2010, 2010, 3461-3466.

2. Wei, X.-F.; Xie, X.-W.; Shimizu, Y.; Kanai, M., Copper(I)-Catalyzed Enantioselective Addition of Enynes to Ketones. J. Am. Chem. Soc. 2017, 139, 4647-4650.

3. Wood, J. L.; Marciasini, L. D.; Vaultier, M.; Pucheault, M., Iron Catalysis and Water: A Synergy for Refunctionalization of Boron. Synlett 2014, 25, 551-555.

4. Wang, C.; Wu, C.; Ge, S., Iron-Catalyzed E-Selective Dehydrogenative Borylation of Vinylarenes with Pinacolborane. ACS Catalysis 2016, 6, 7585-7589.

5. Itoh, T.; Shimizu, Y.; Kanai, M., Copper-Catalyzed Regio- and Stereoselective Synthesis of Trialkylsubstituted Alkenylboronates from Unactivated Internal Alkynes . J. Am. Chem. Soc. 2016, 138, 75287531.

6. $\quad$ Nishihara, Y.; Inoue, E.; Noyori, S.; Ogawa, D.; Okada, Y.; Iwasaki, M.; Takagi, K., Synthesis of unsymmetrically disubstituted ethynes by the palladium/copper(I)-cocatalyzed sila-Sonogashira-Hagihara coupling reactions of alkynylsilanes with aryl iodides, bromides, and chlorides through a direct activation of a carbon-silicon bond. Tetrahedron 2012, 68, 4869-4881.

7. Yu, S.; Wu, C.; Ge, S., Cobalt-Catalyzed Asymmetric Hydroboration/Cyclization of 1,6-Enynes with Pinacolborane. J. Am. Chem. Soc. 2017, 139, 6526-6529. 
NMR Spectra $\left({ }^{1} \mathrm{H},\left\{{ }^{1} \mathrm{H}\right\}{ }^{13} \mathrm{C}\right.$ and $\left.{ }^{19} \mathrm{~F}\right)$
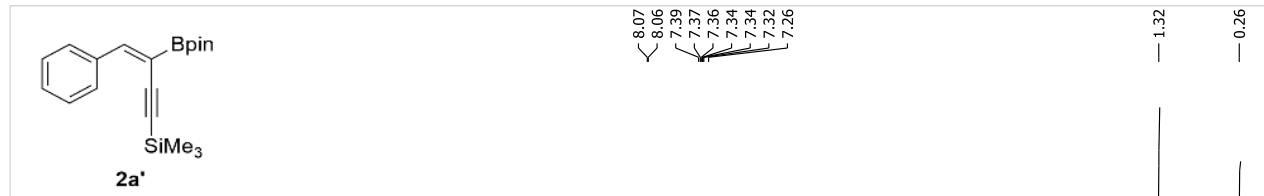

$2 a^{\circ}$
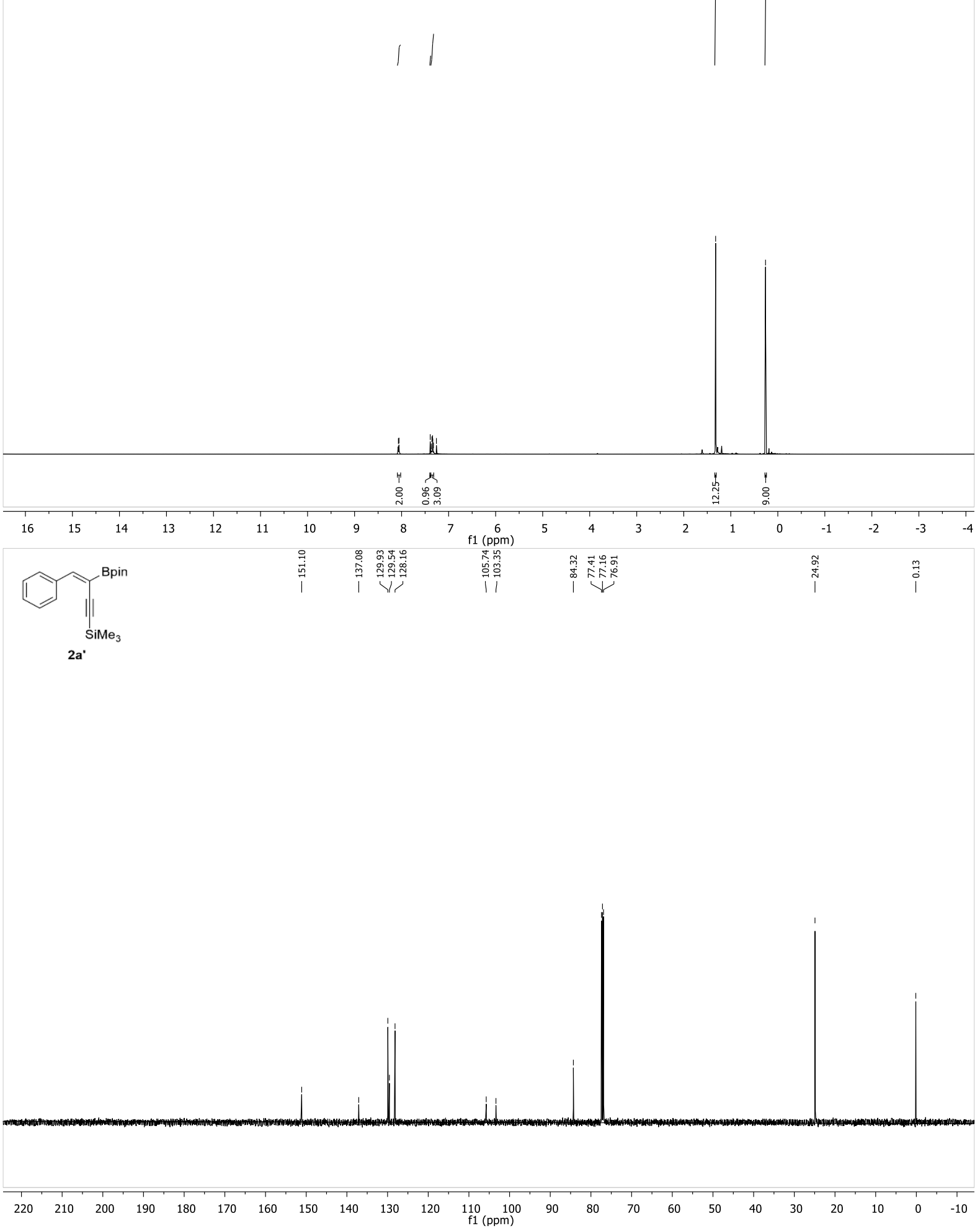


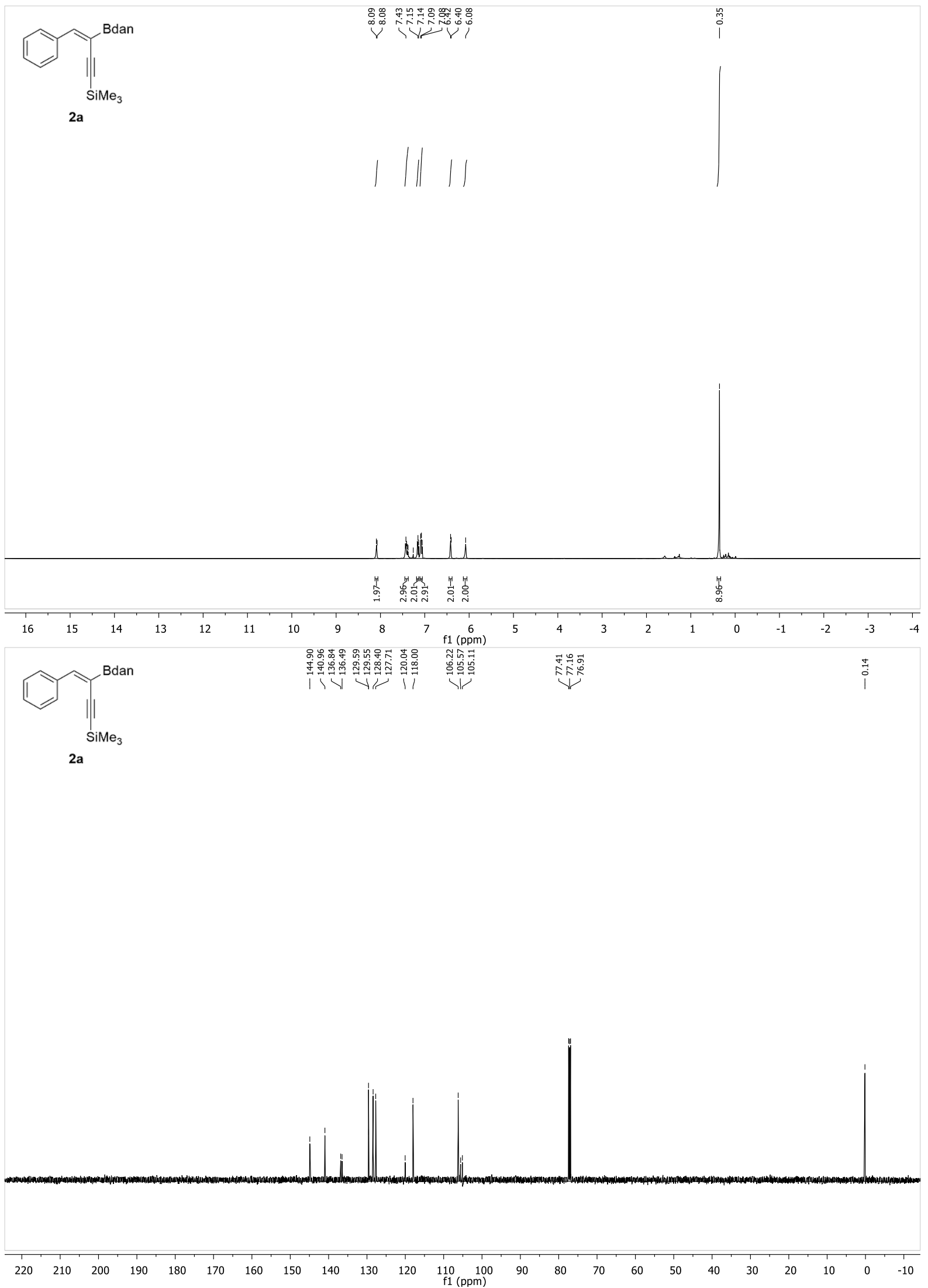




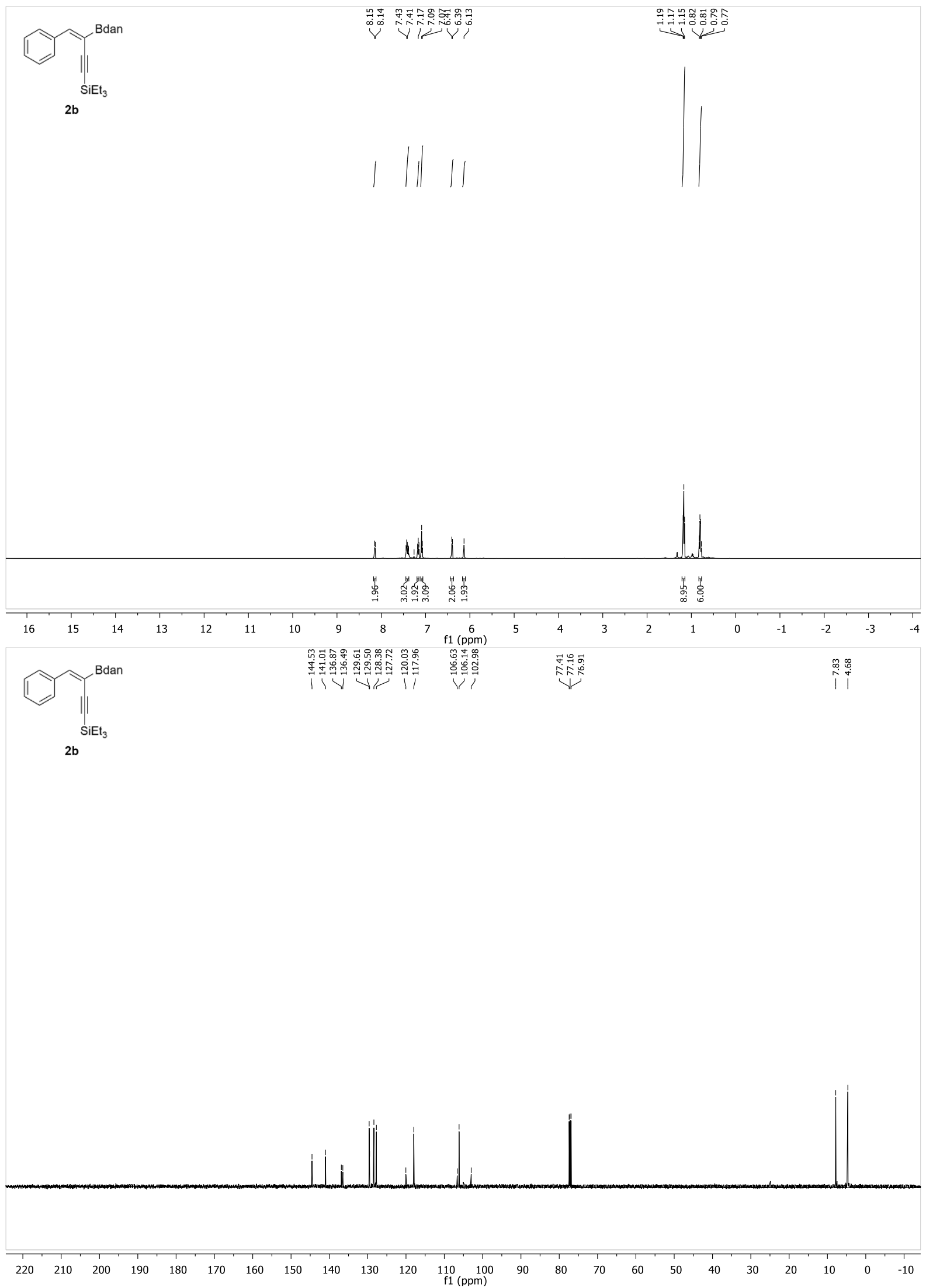



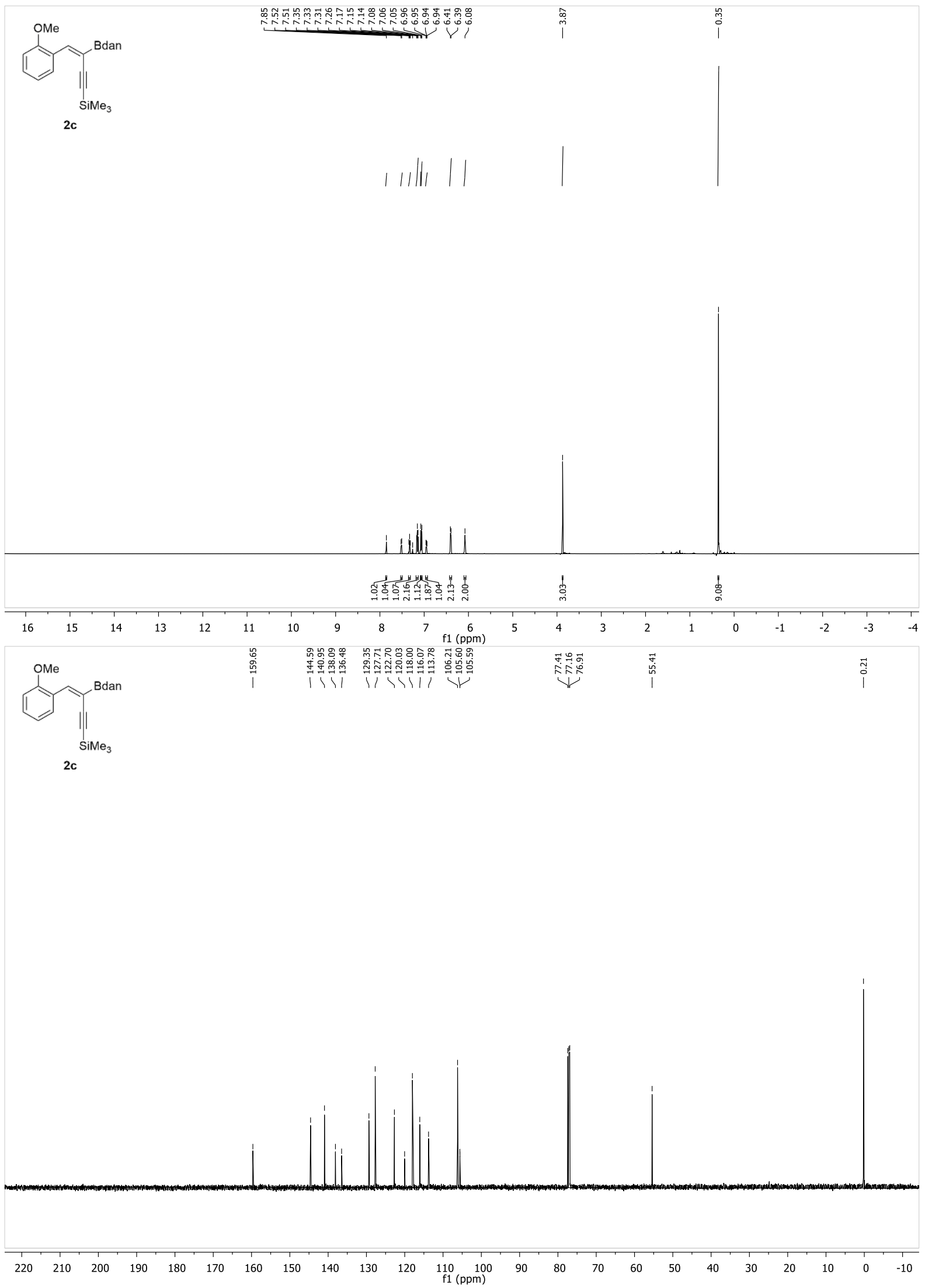


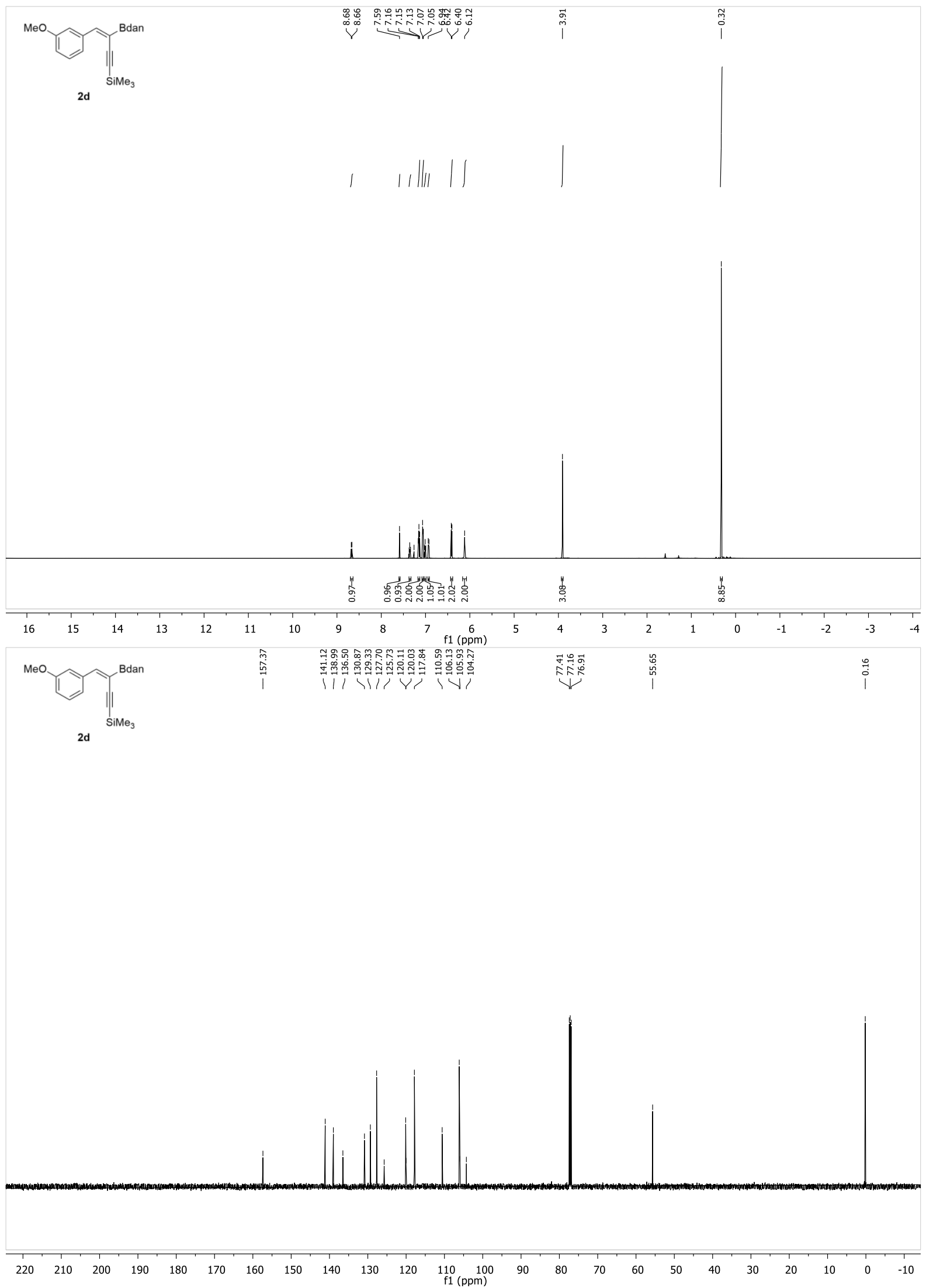


Sang, Wu, Phua and Ge, Supporting Information

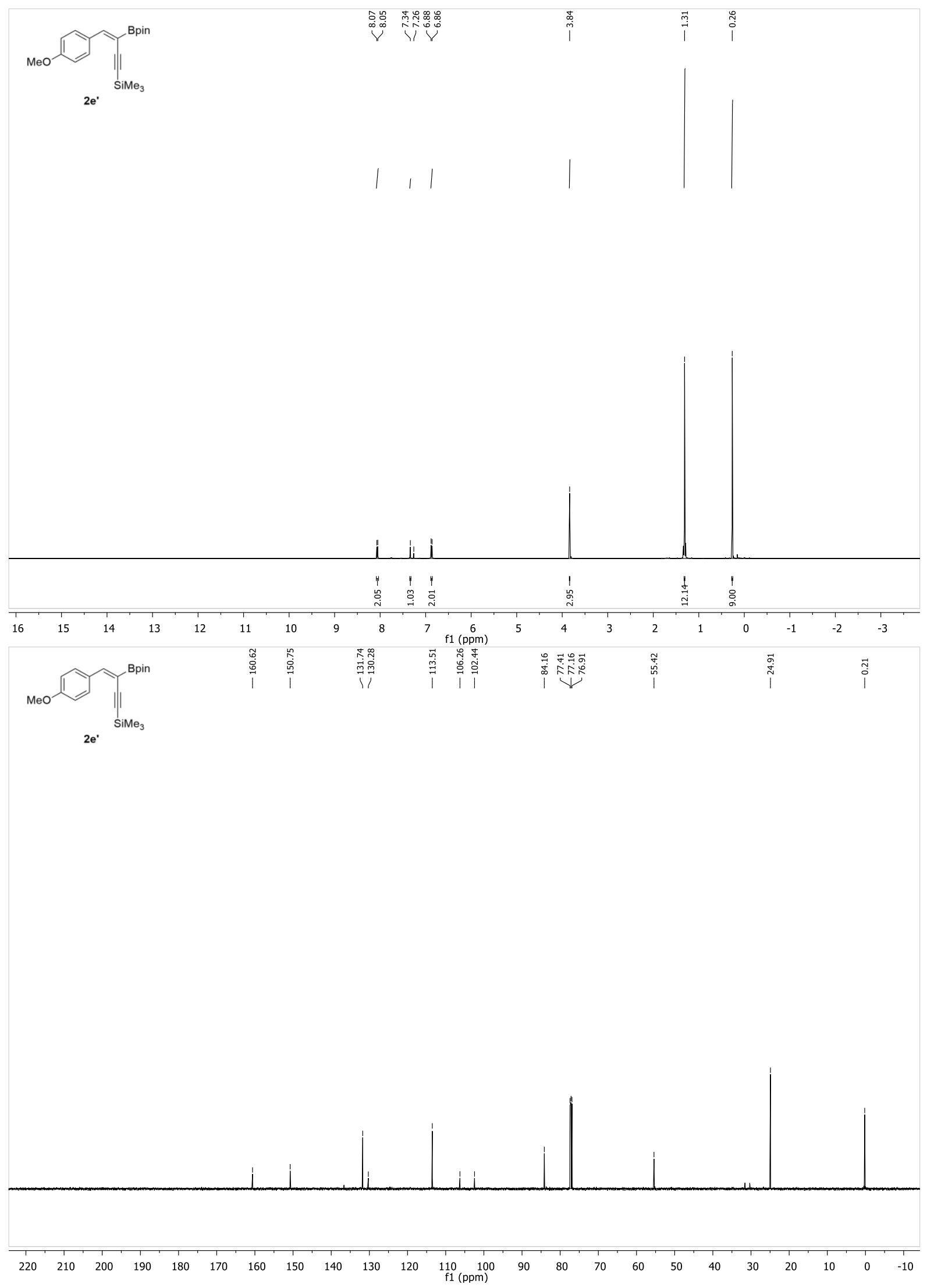

S28 


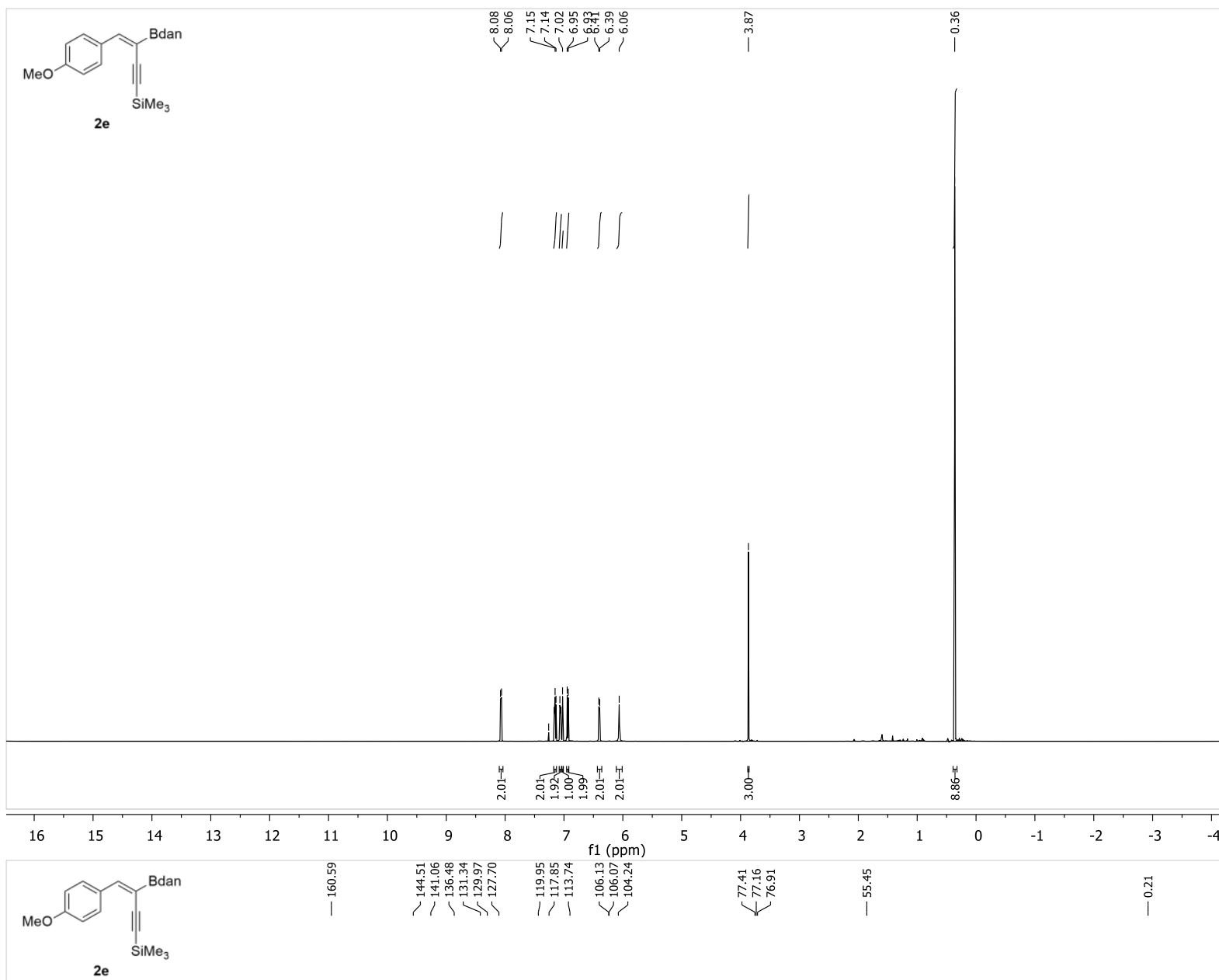

$2 \mathrm{e}$

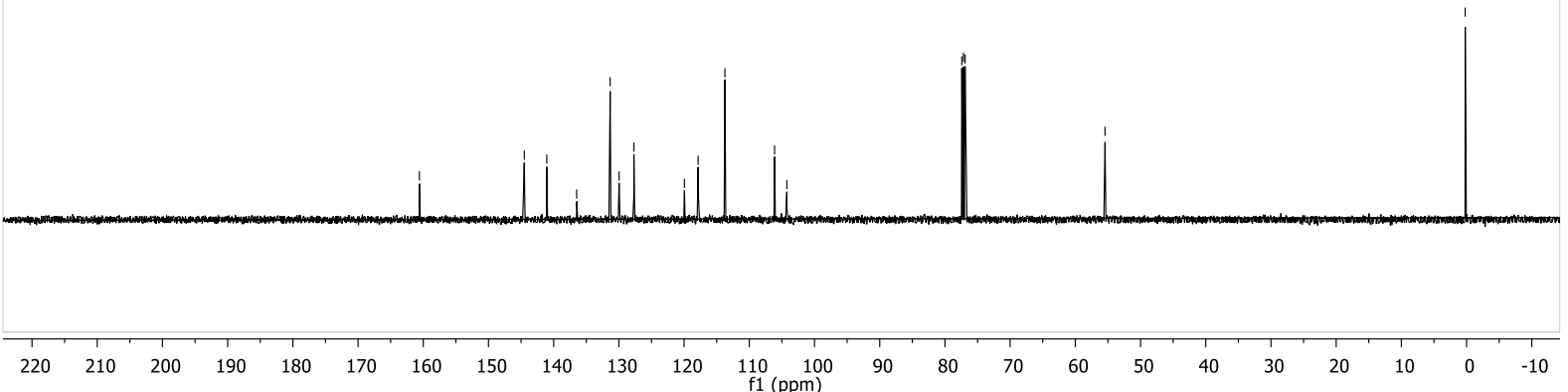




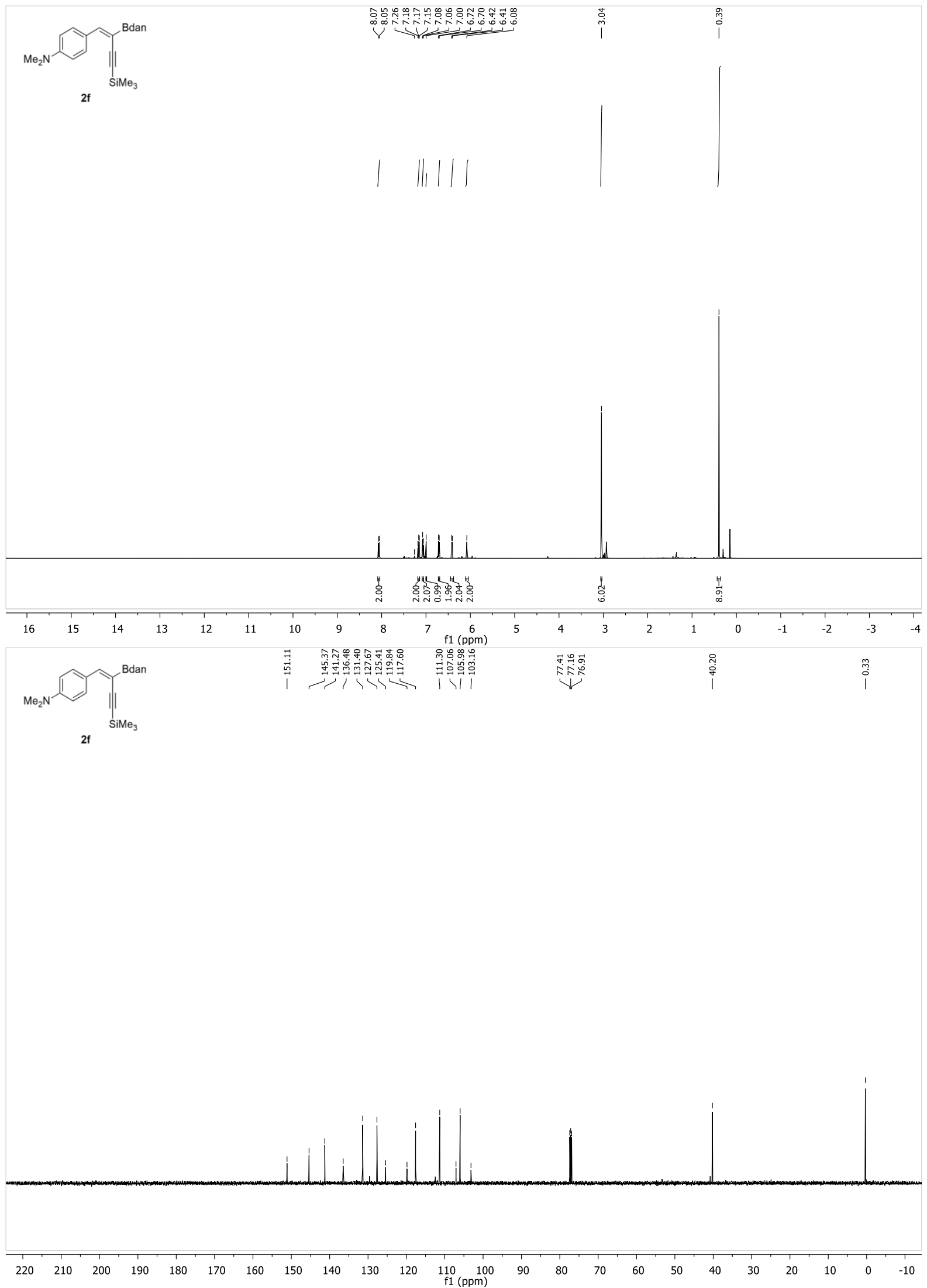



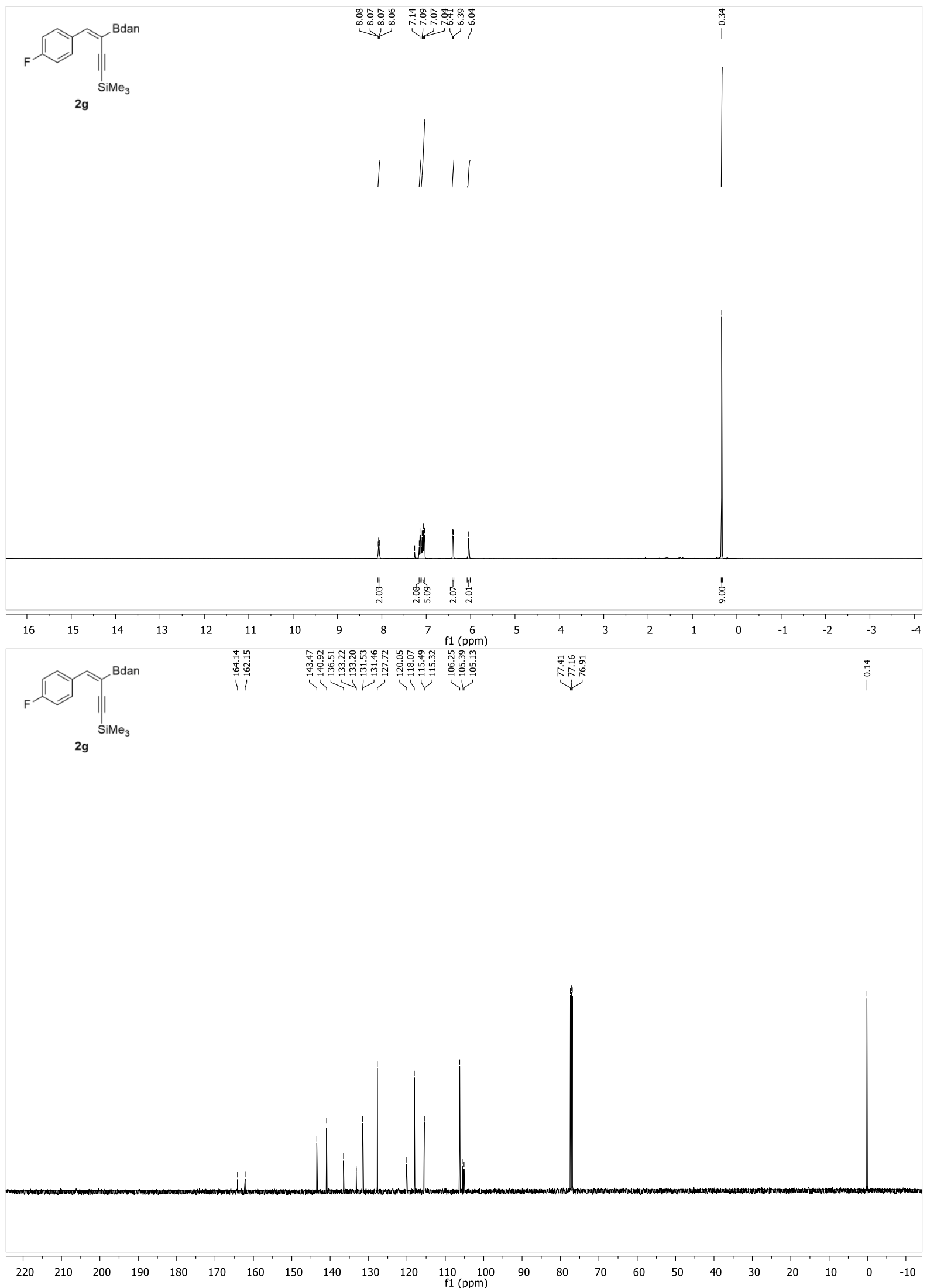


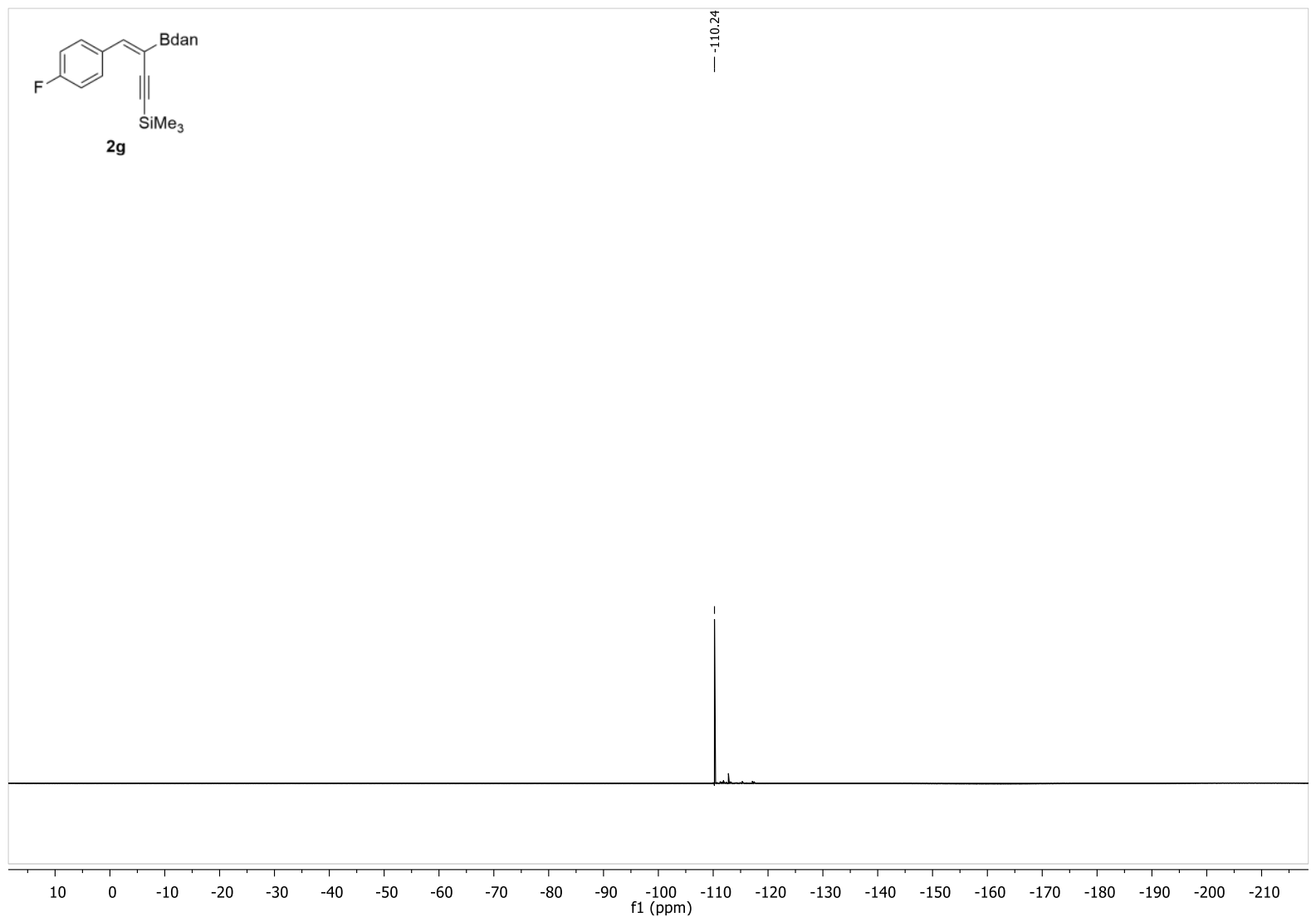



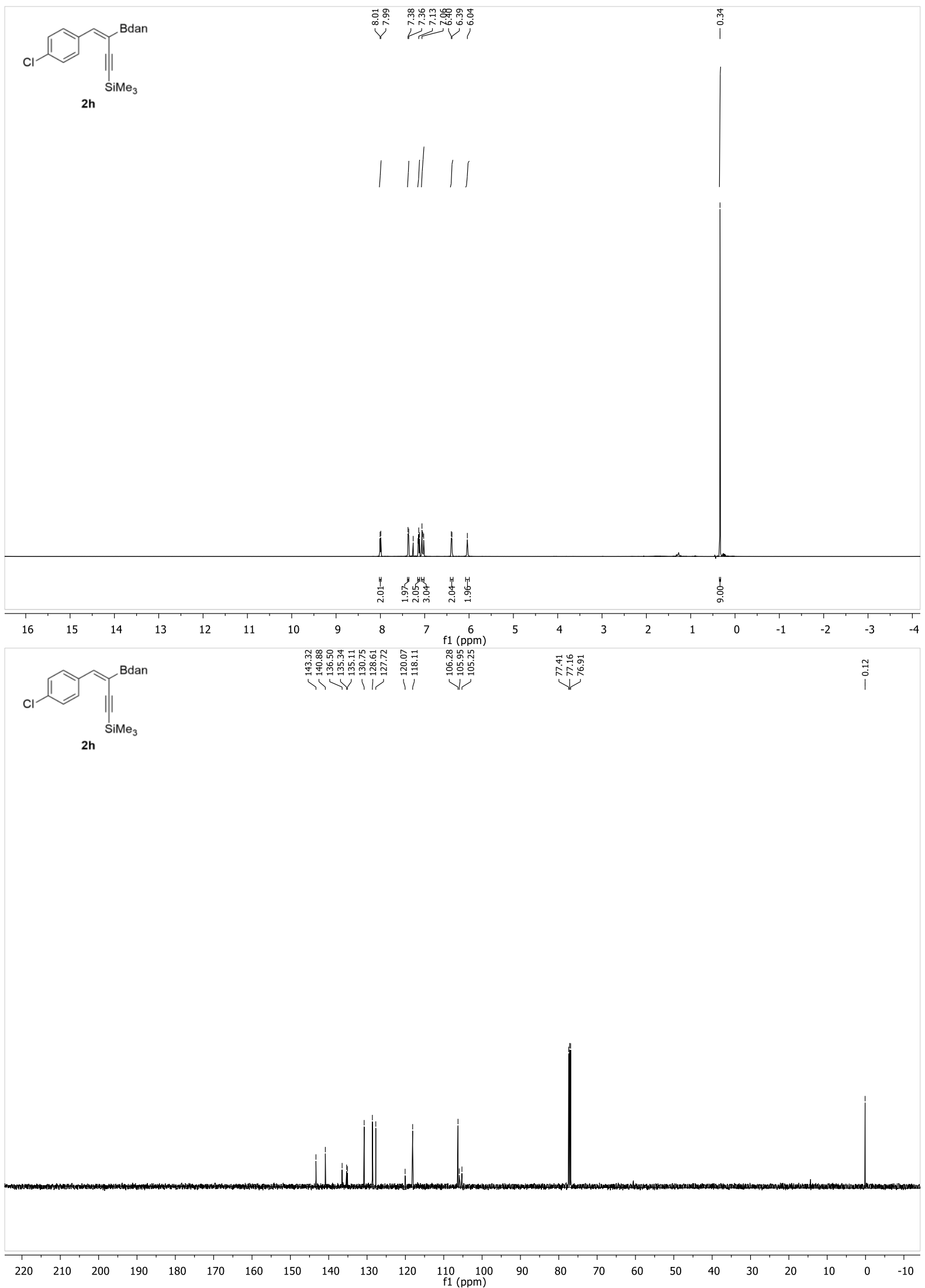


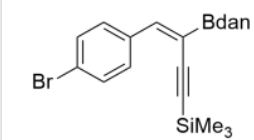

2i

Lill

ili

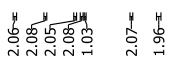

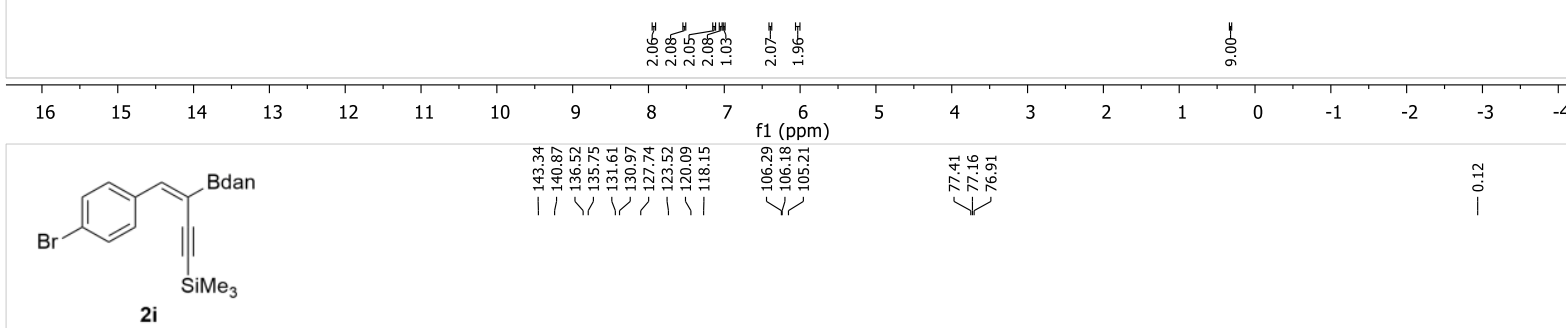

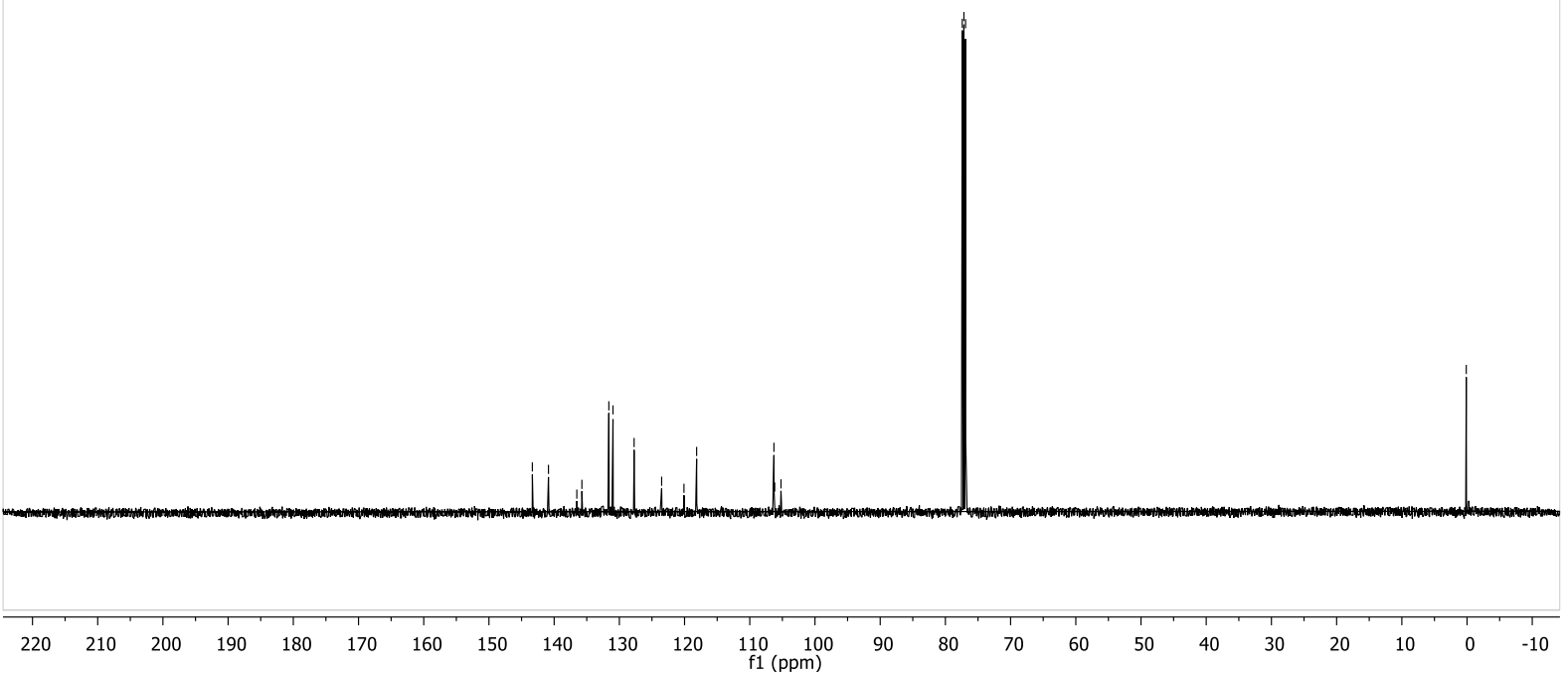




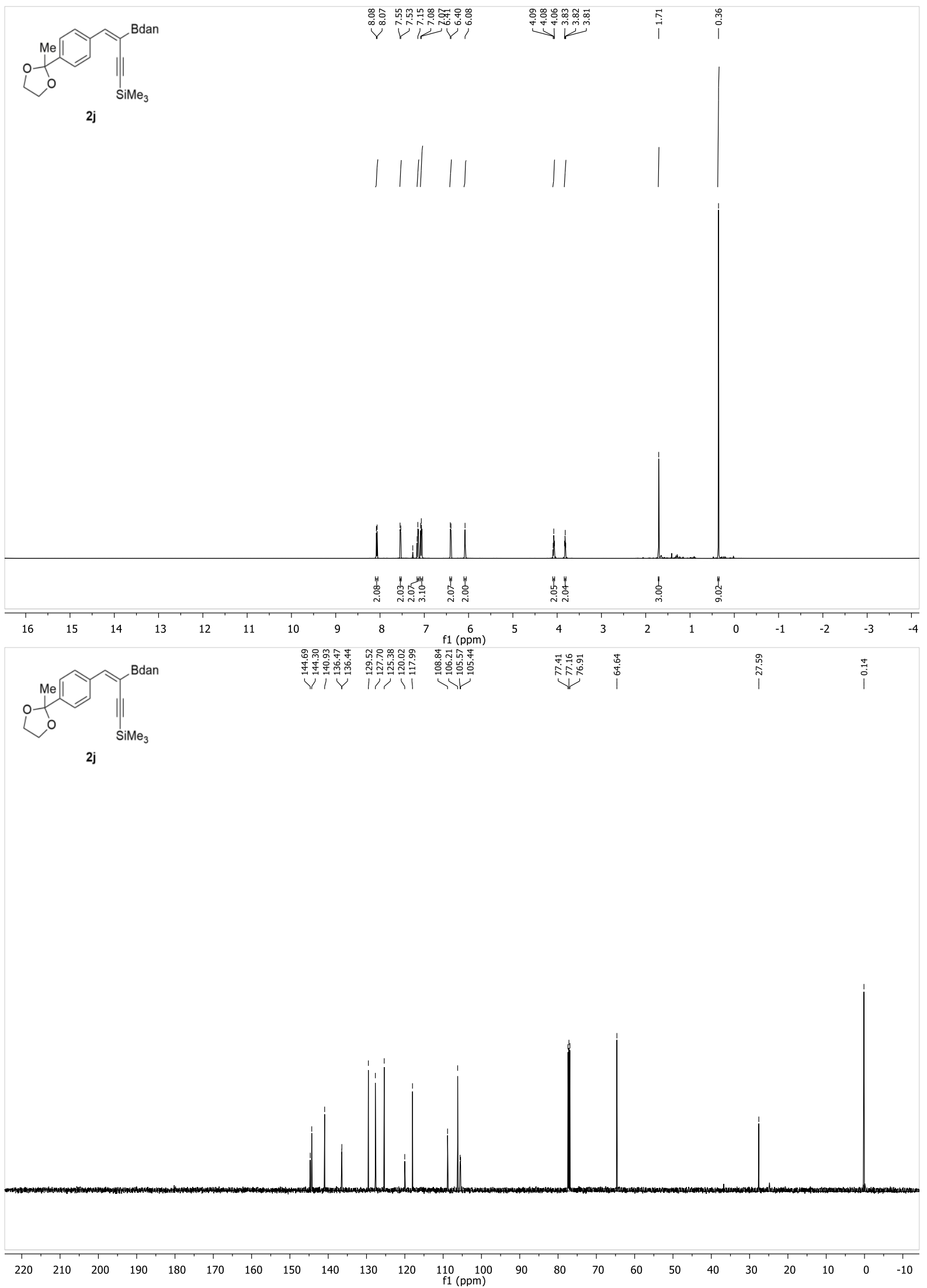




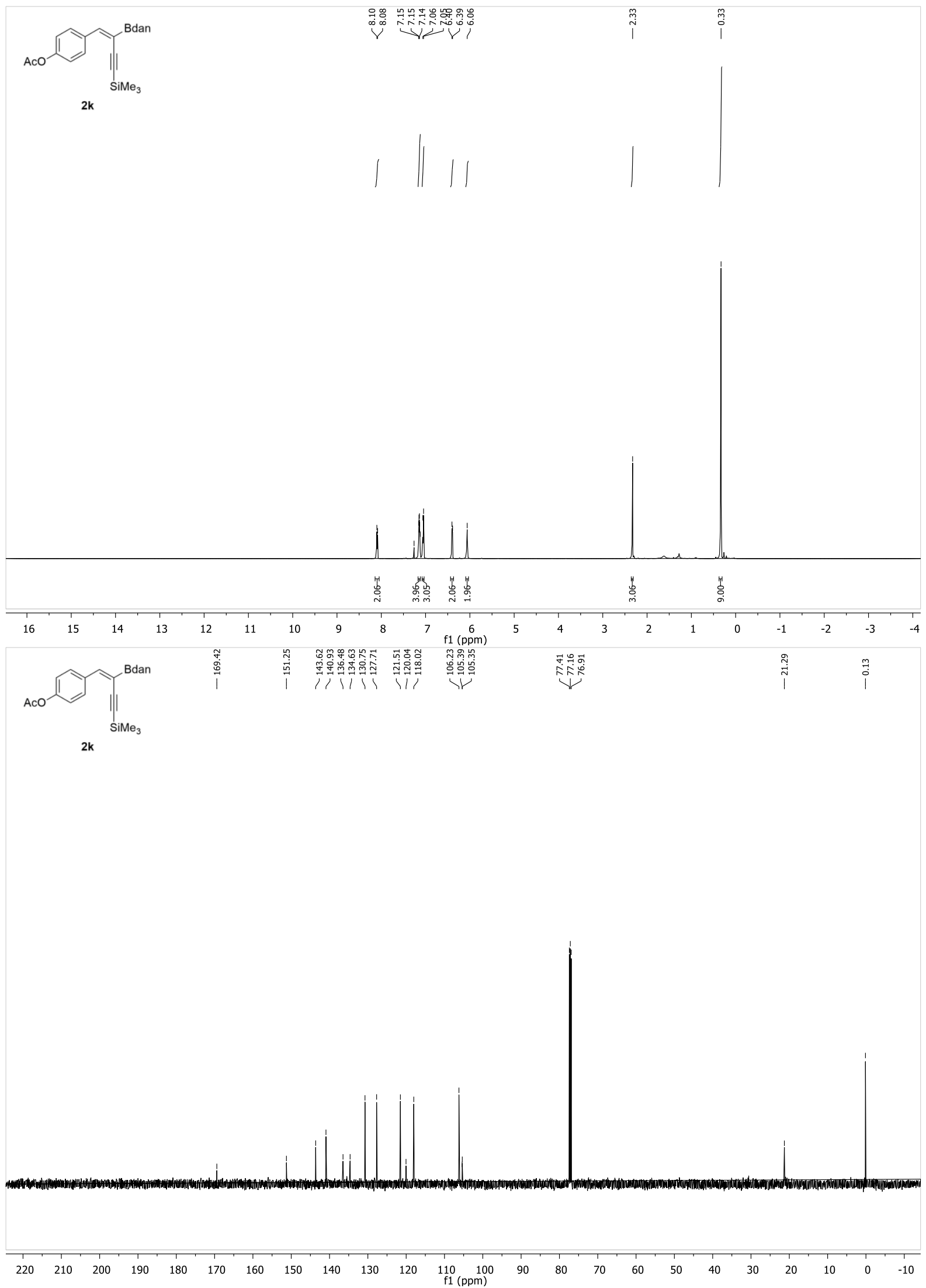




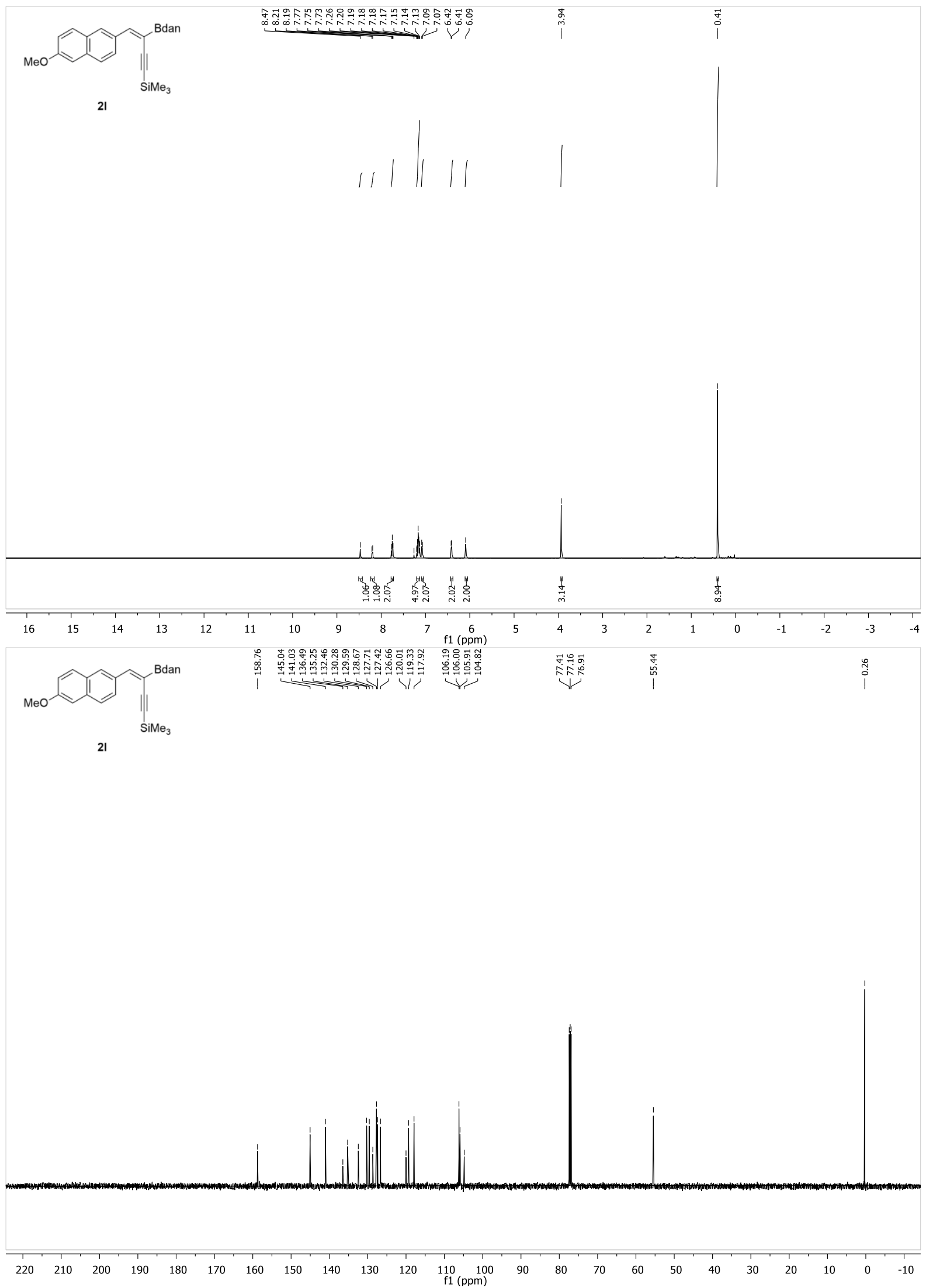




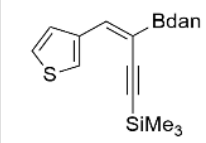

$2 m$

111111
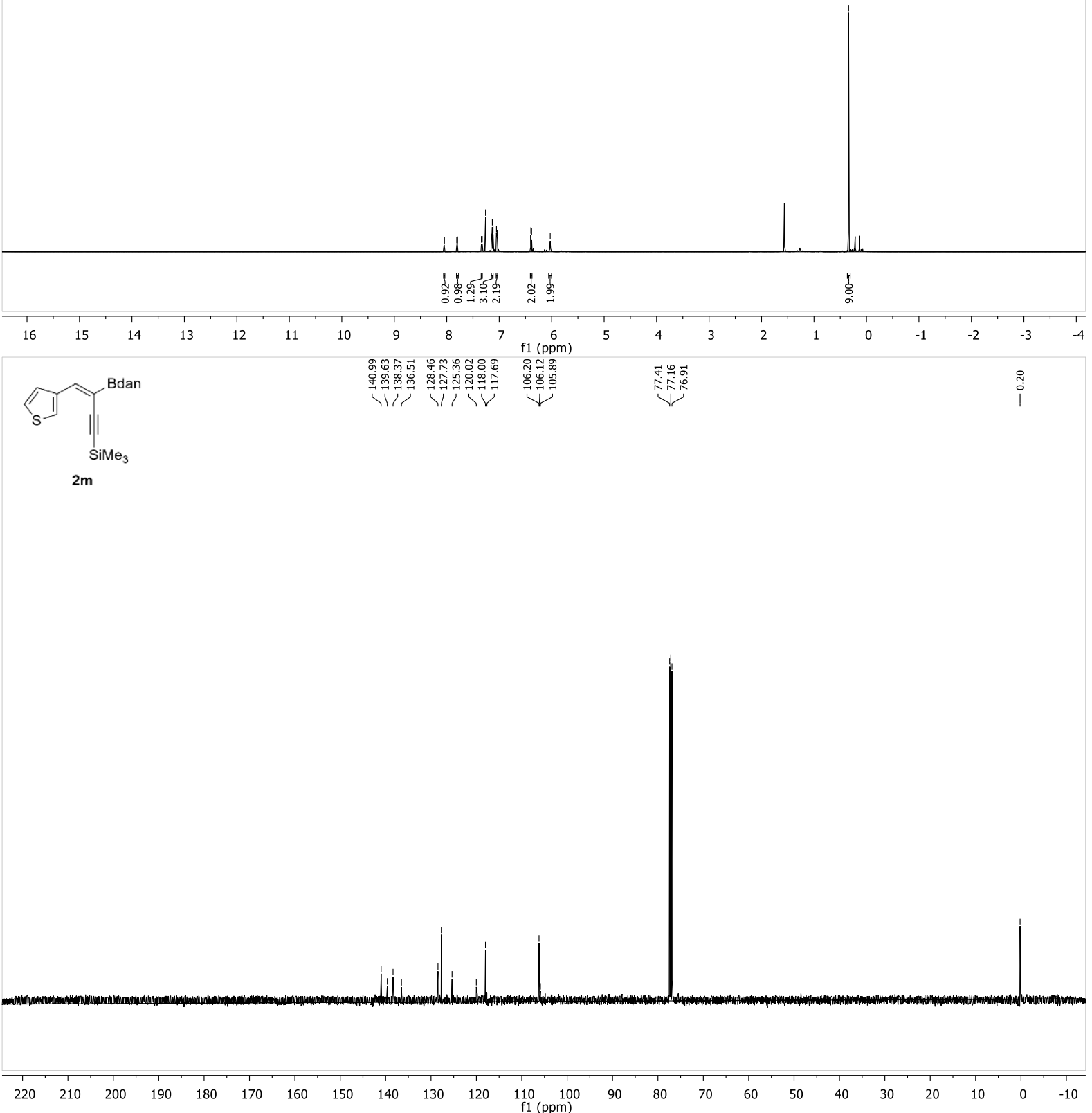


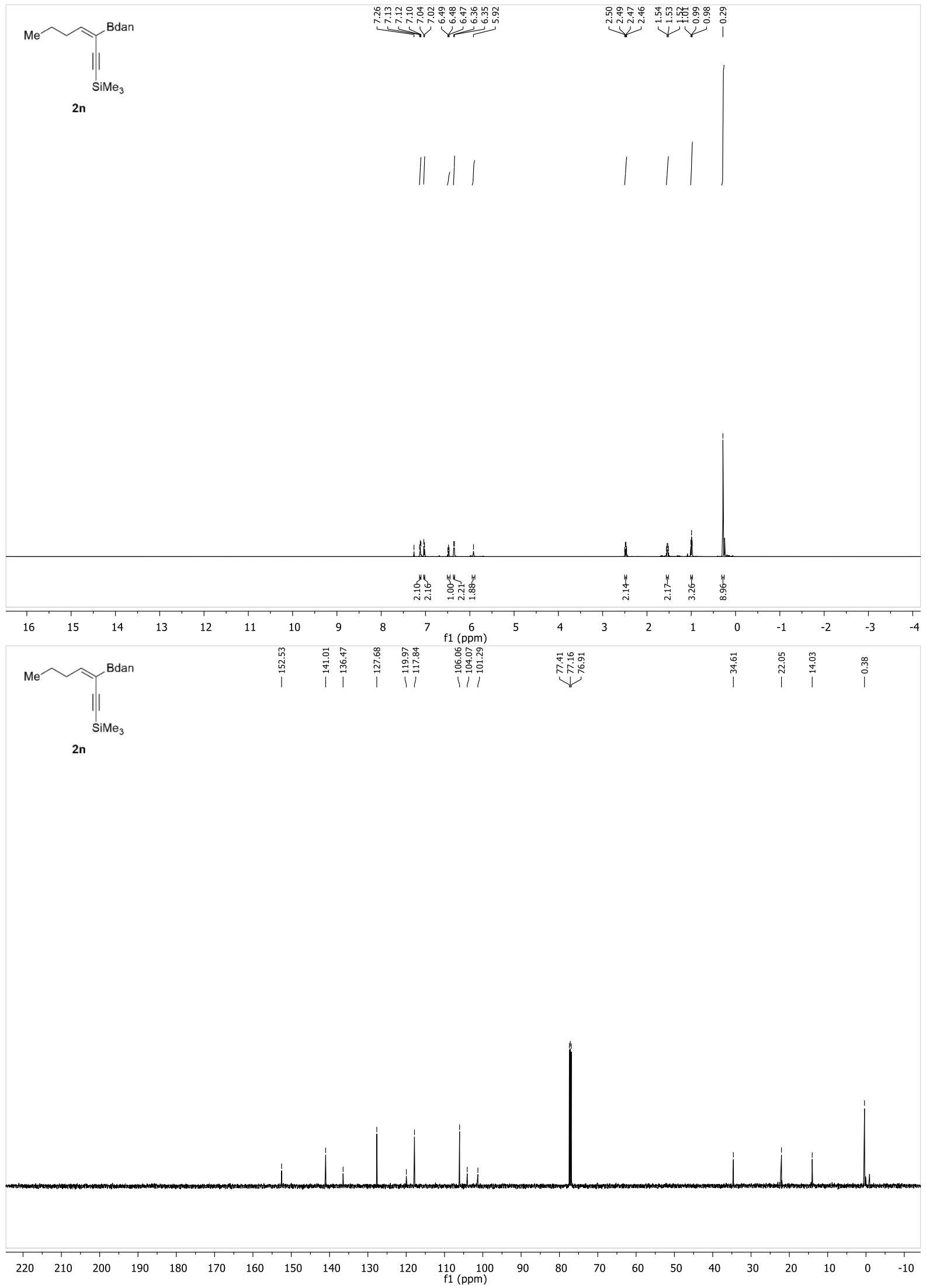




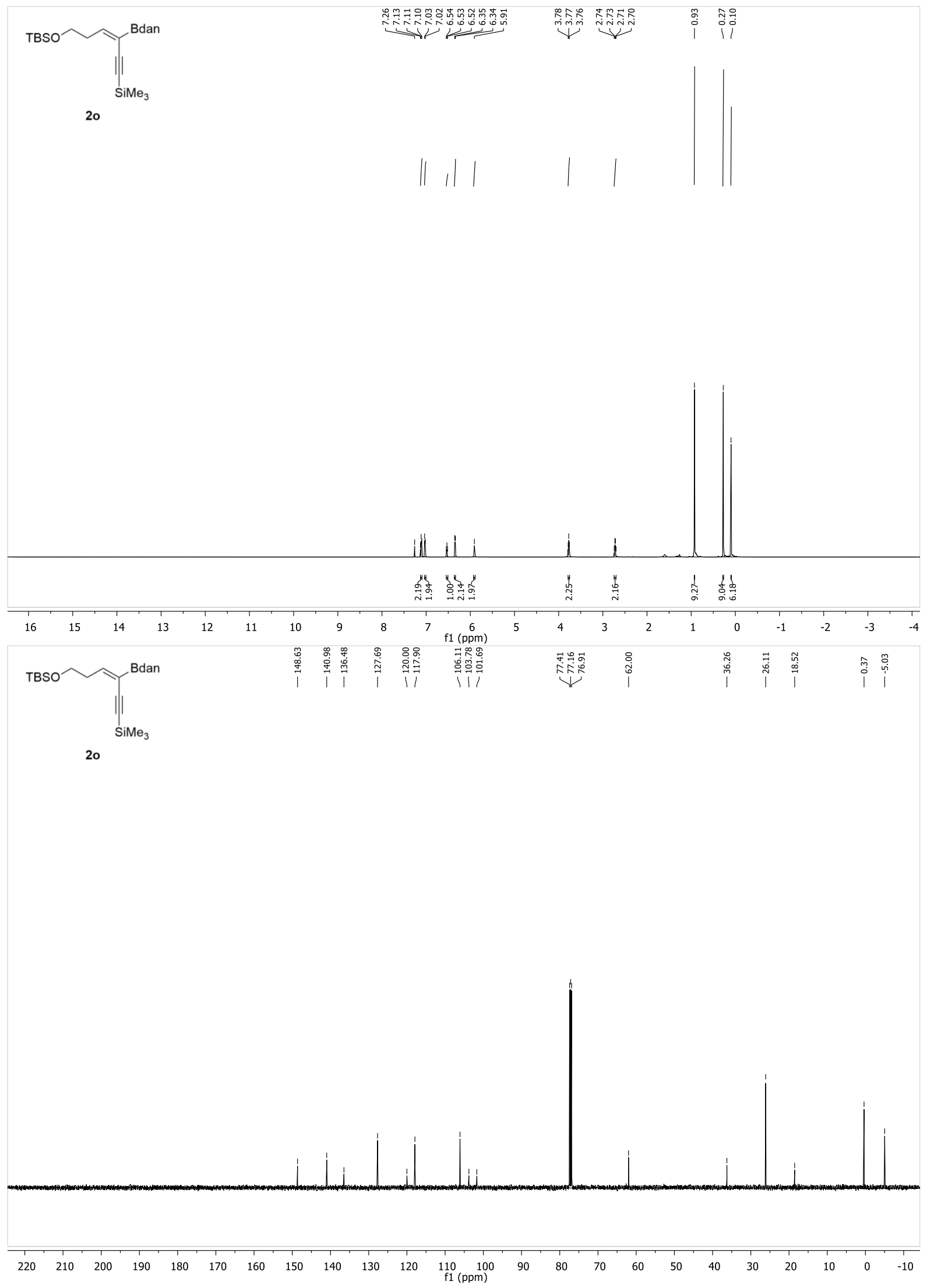



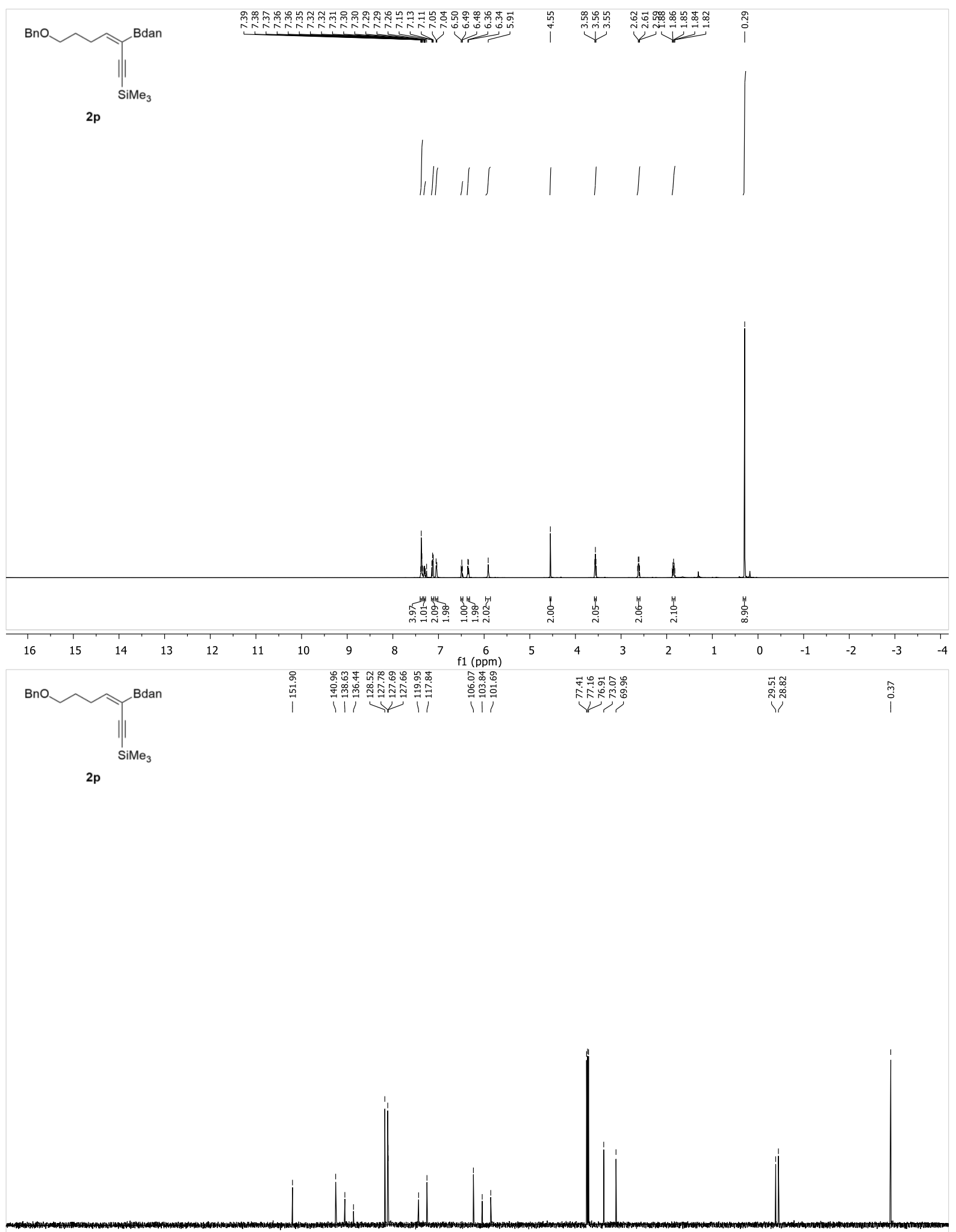

$\begin{array}{lllllllllllllllllllllllll}220 & 210 & 200 & 190 & 180 & 170 & 160 & 150 & 140 & 130 & 120 & 110 & 100 & 90 & 80 & 70 & 60 & 50 & 40 & 30 & 20 & 10 & 0 & -10\end{array}$ 


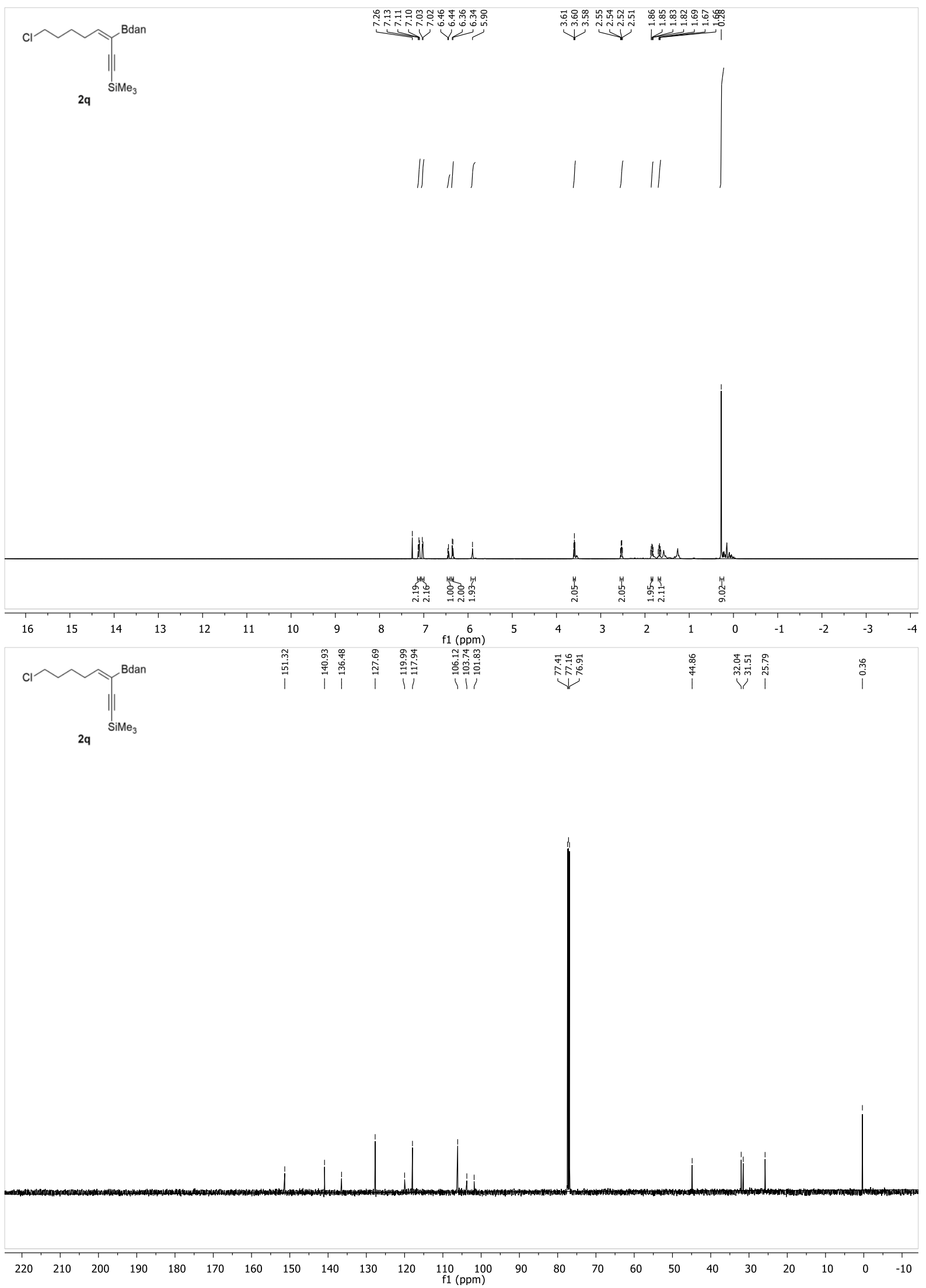




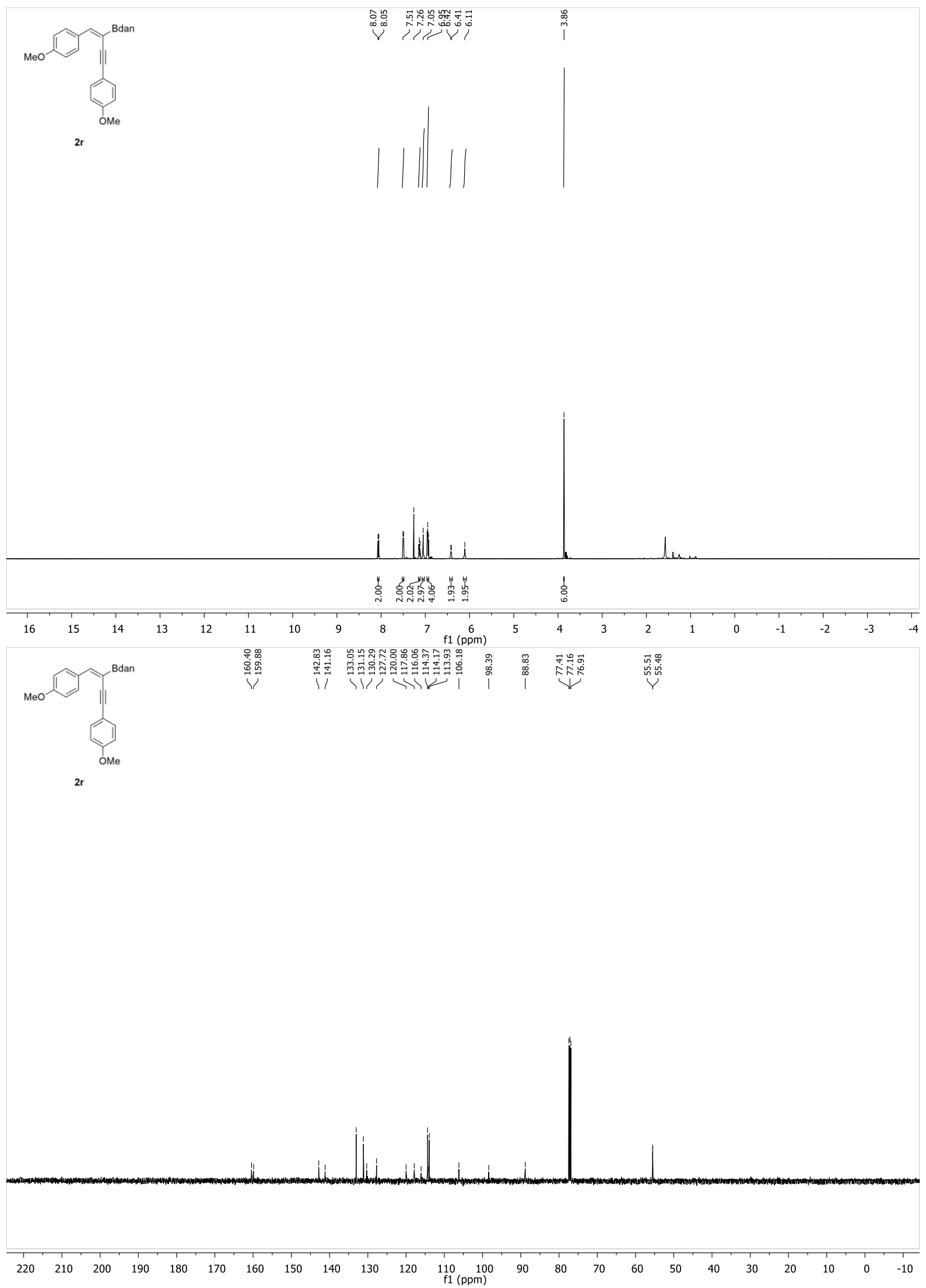




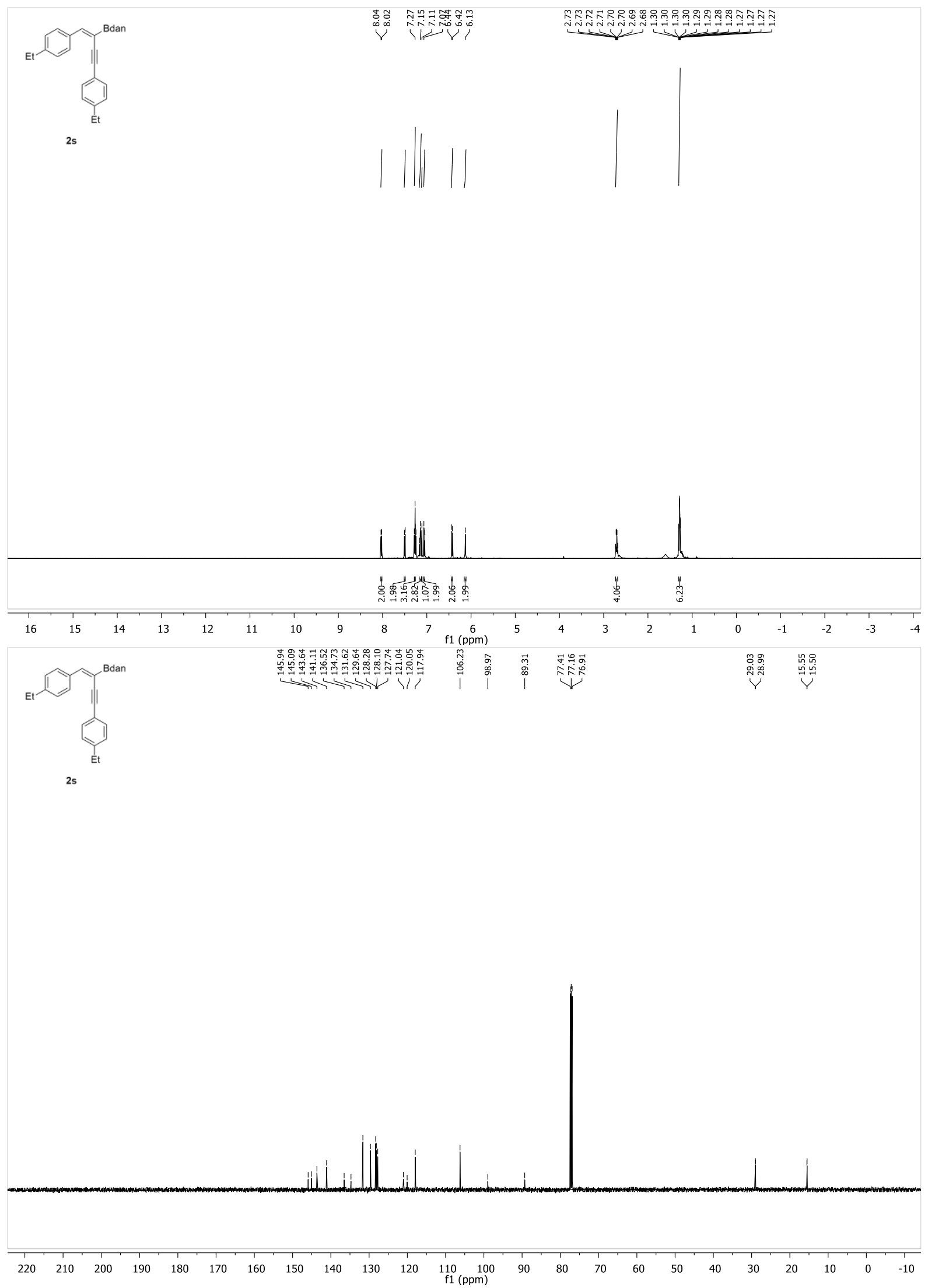




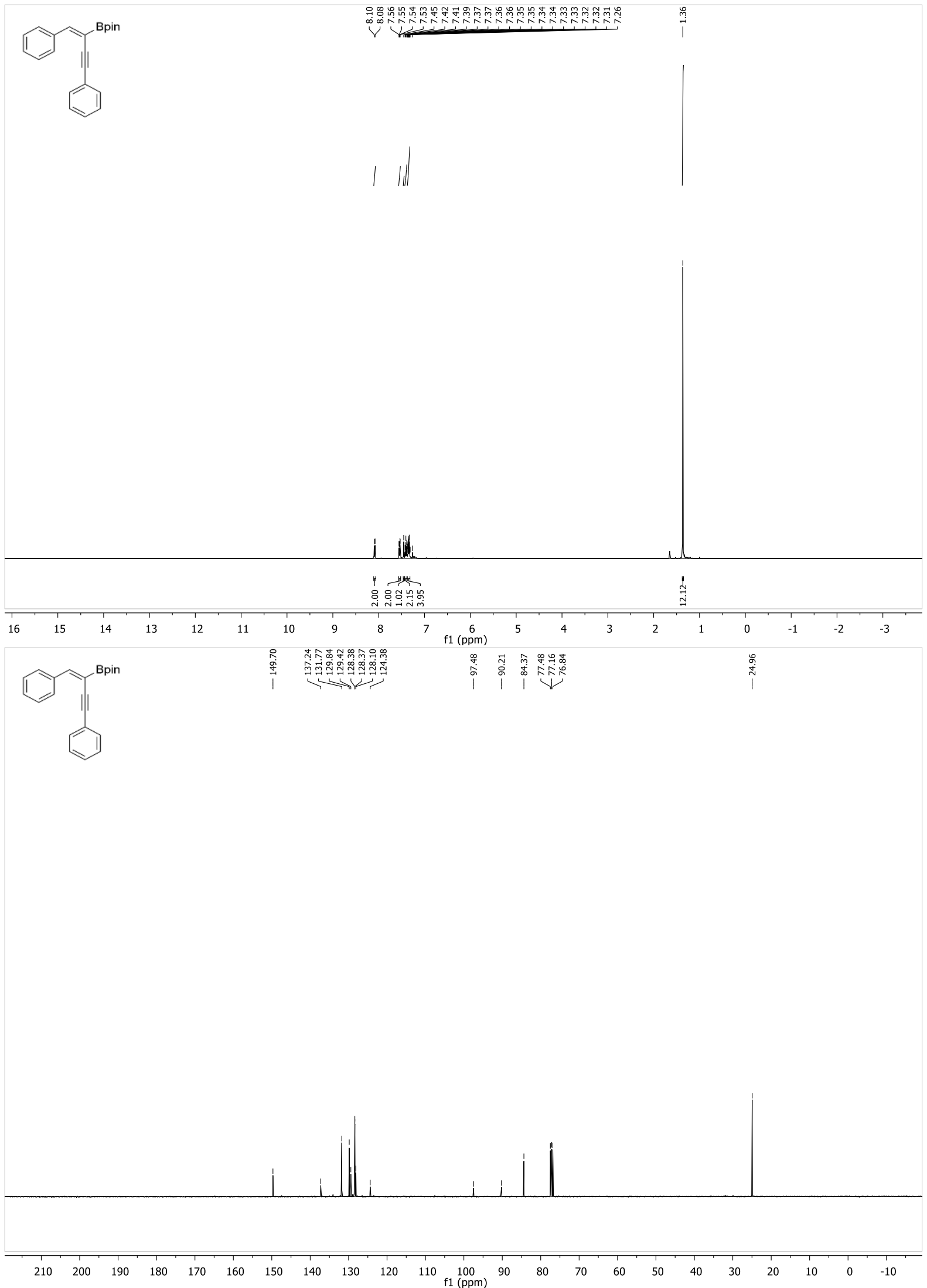



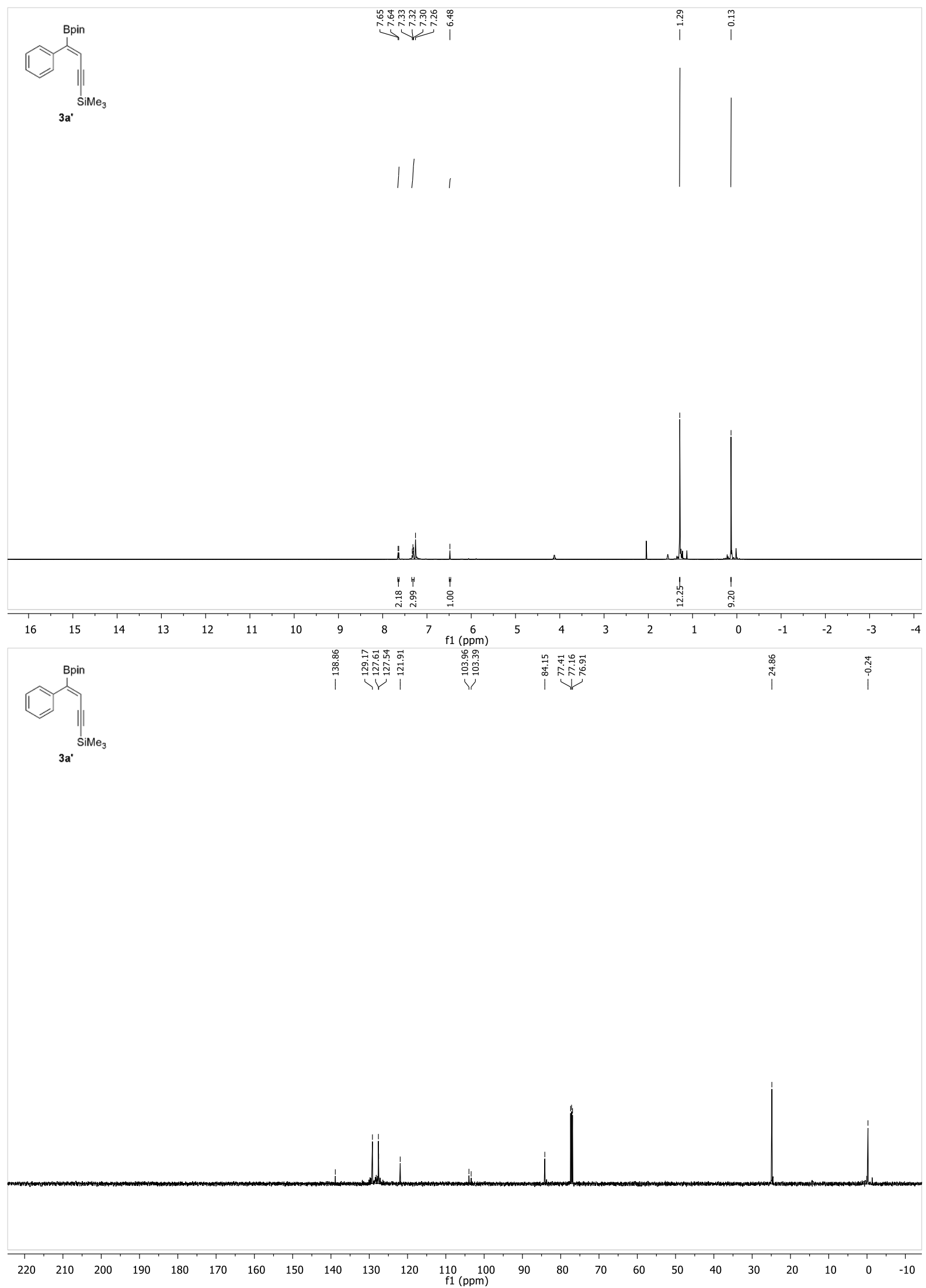


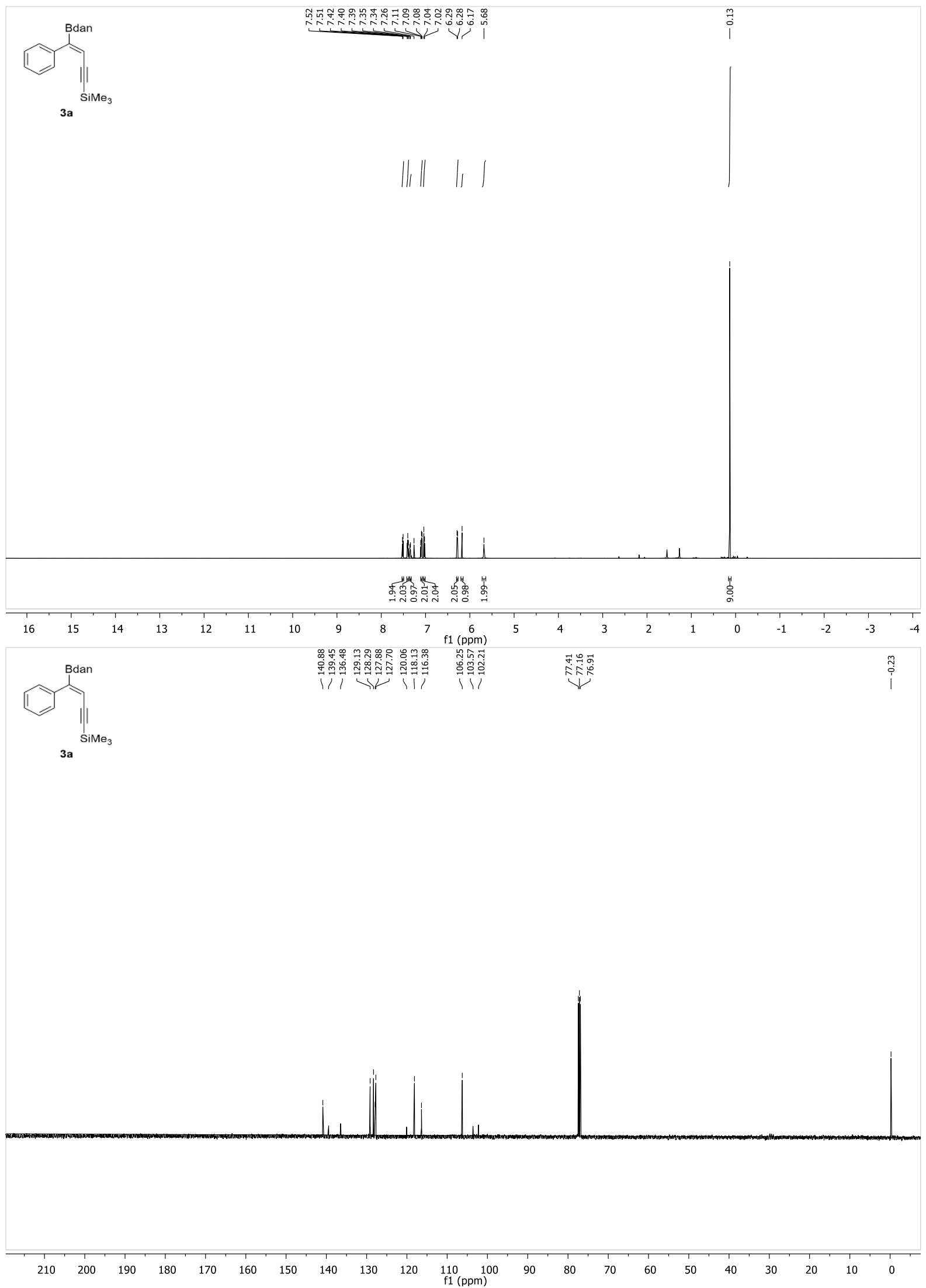




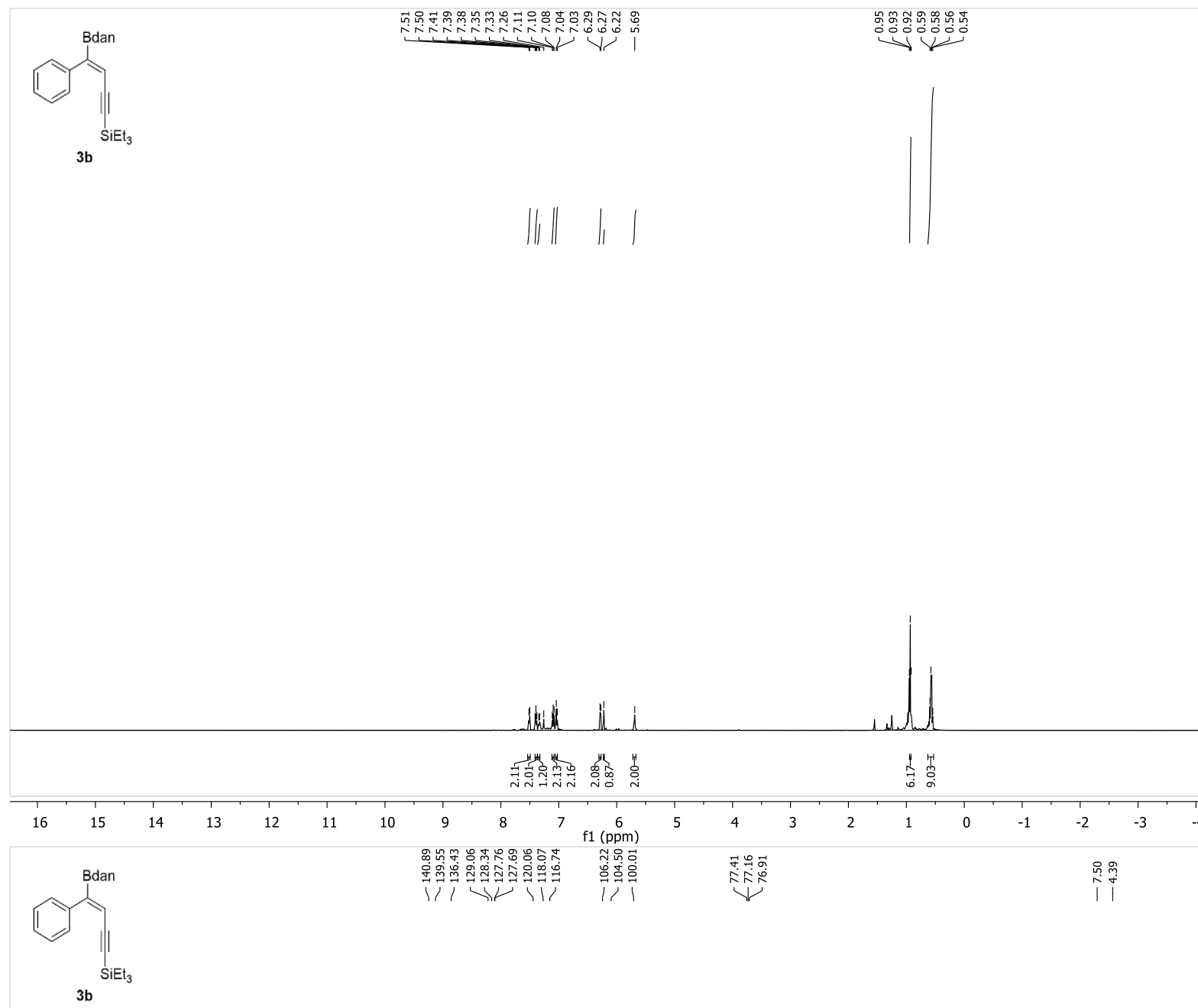

3b

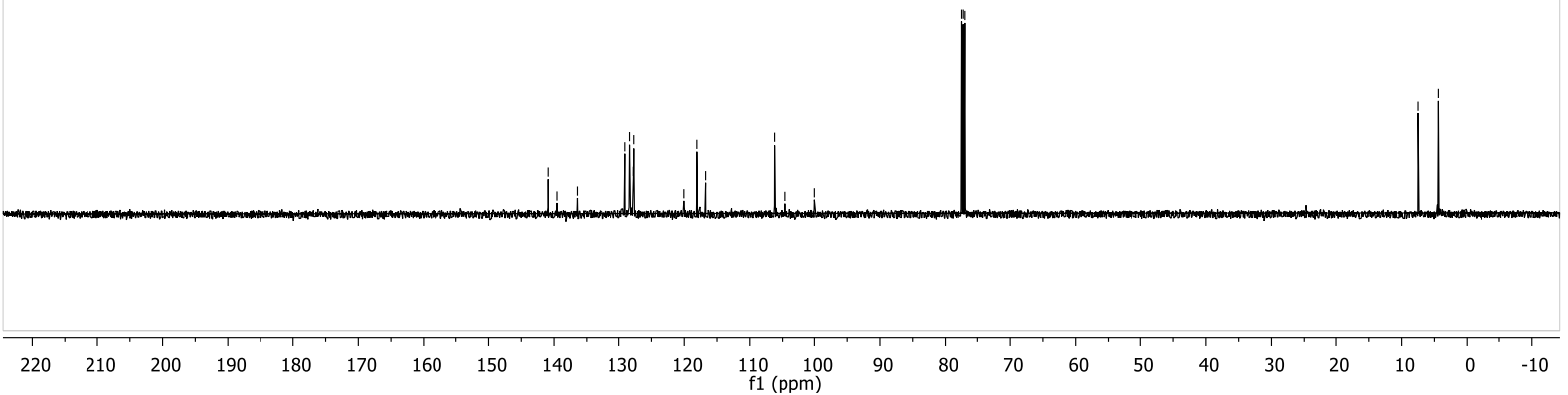



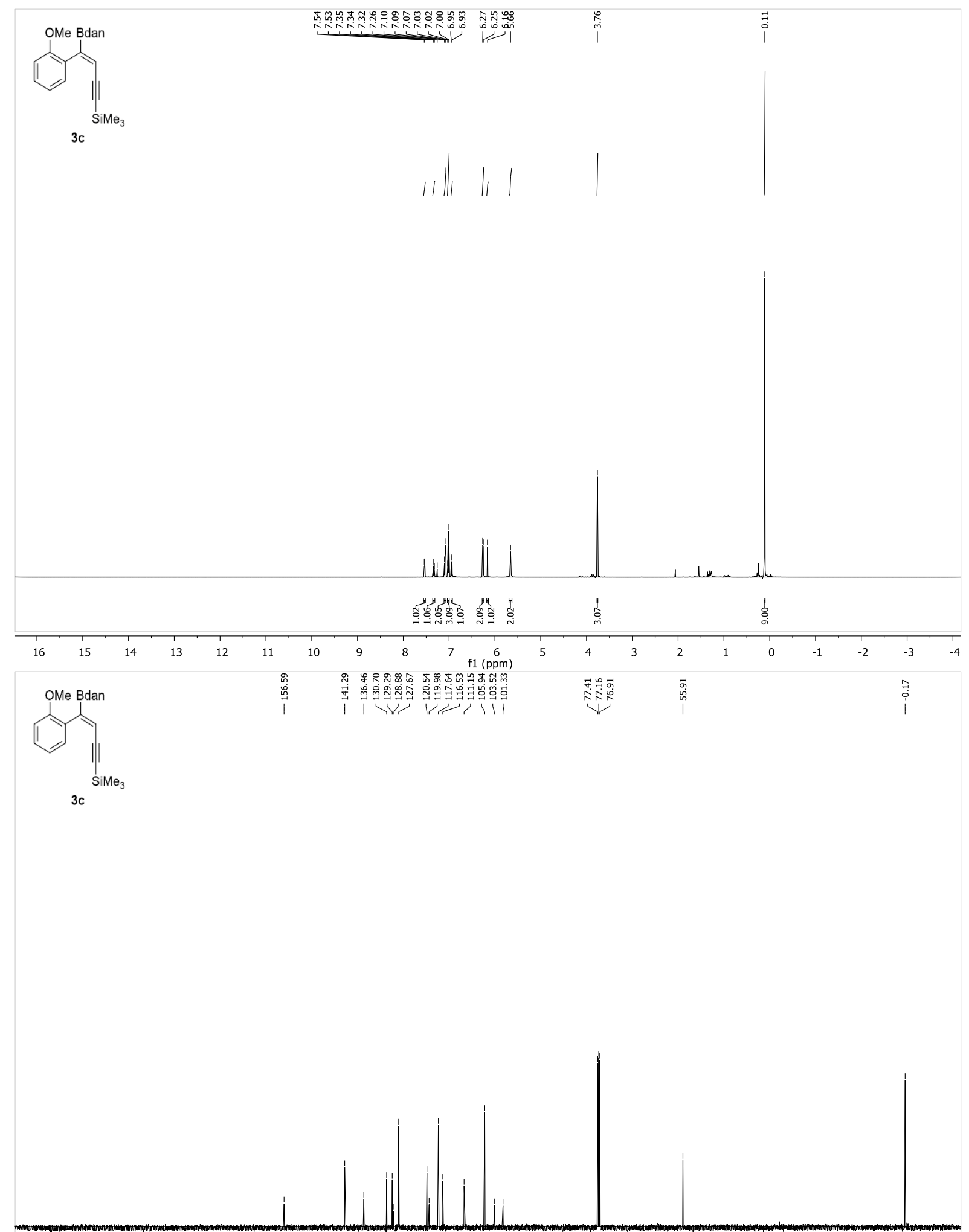

$\begin{array}{llllllllllllllllllllllllll}220 & 210 & 200 & 190 & 180 & 170 & 160 & 150 & 140 & 130 & 120 & 110 & 100 & 90 & 80 & 70 & 60 & 50 & 40 & 30 & 20 & 10 & 0 & -10\end{array}$ 

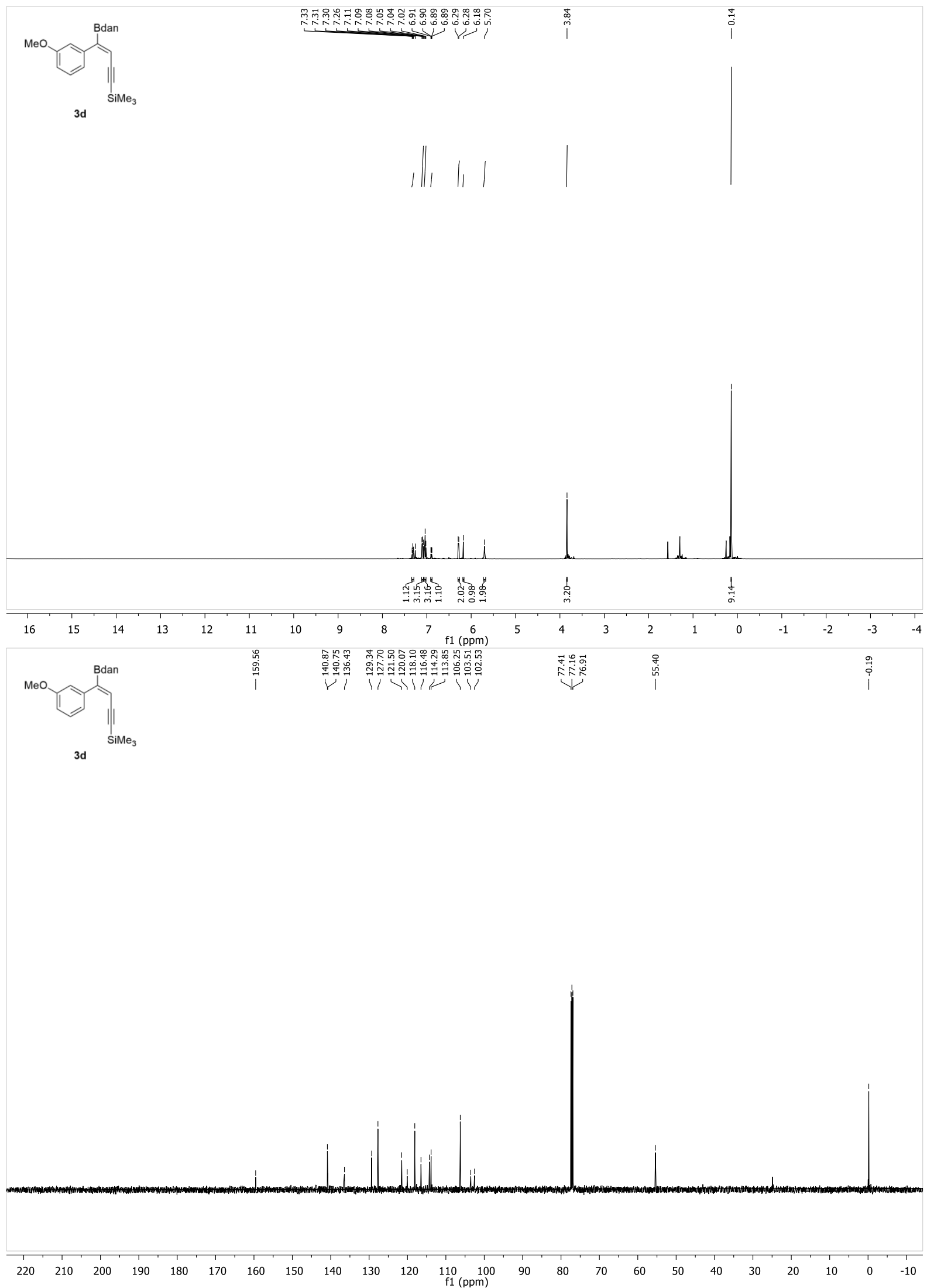


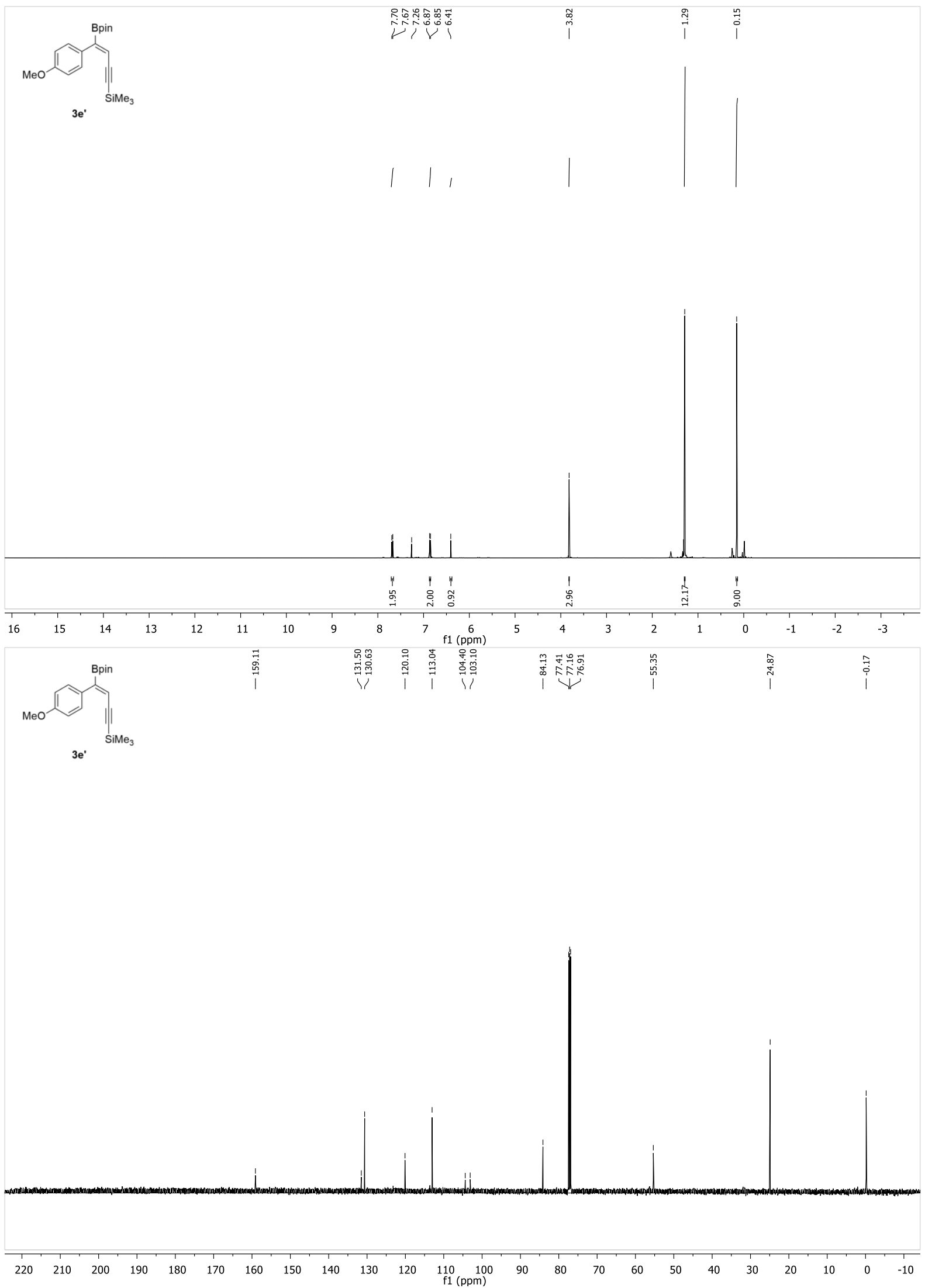



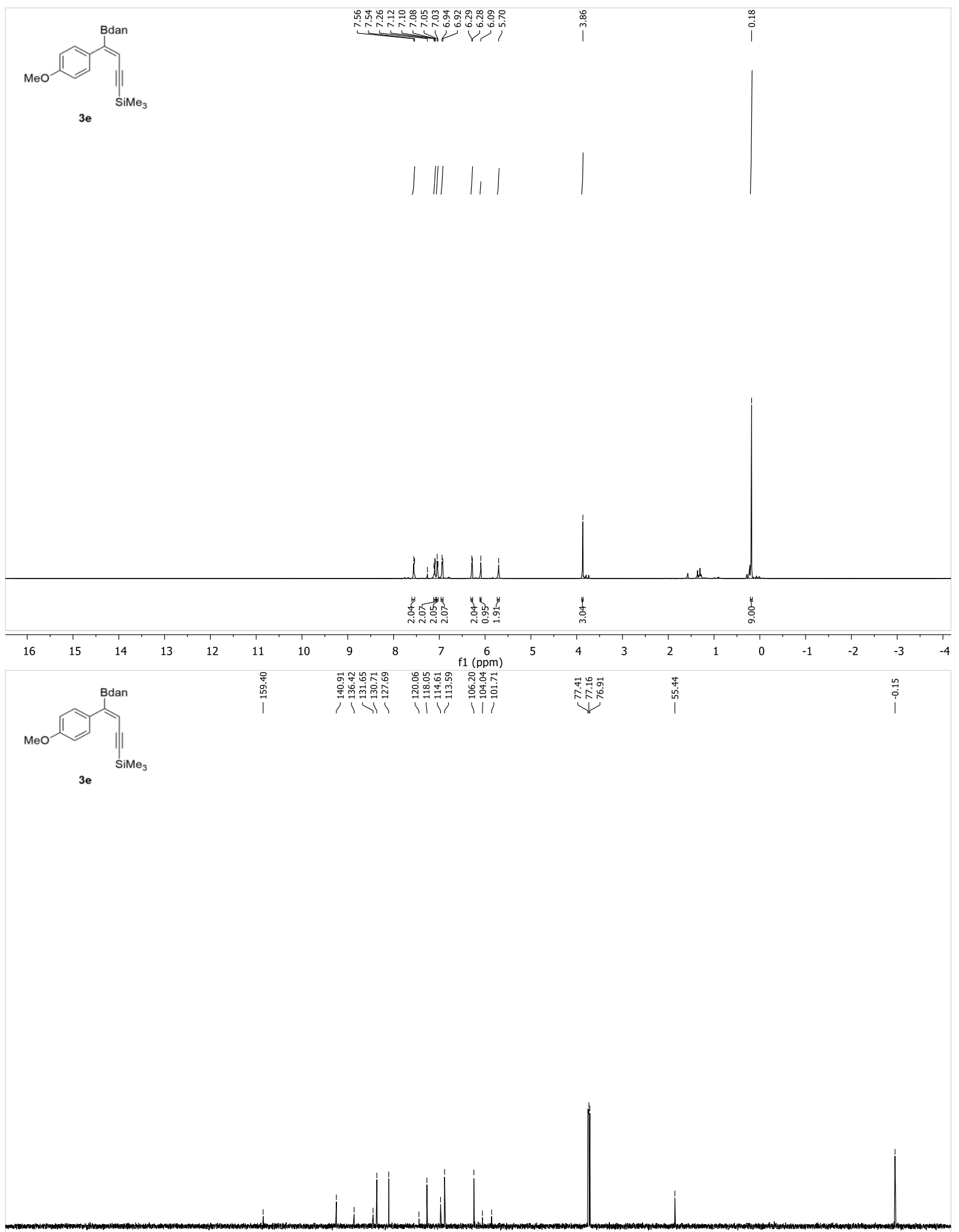

$\begin{array}{lllllllllllllllllllllllllllll}220 & 210 & 200 & 190 & 180 & 170 & 160 & 150 & 140 & 130 & 120 & 110 & 100 & 90 & 80 & 70 & 60 & 50 & 40 & 30 & 20 & 10 & 0 & -10\end{array}$ 

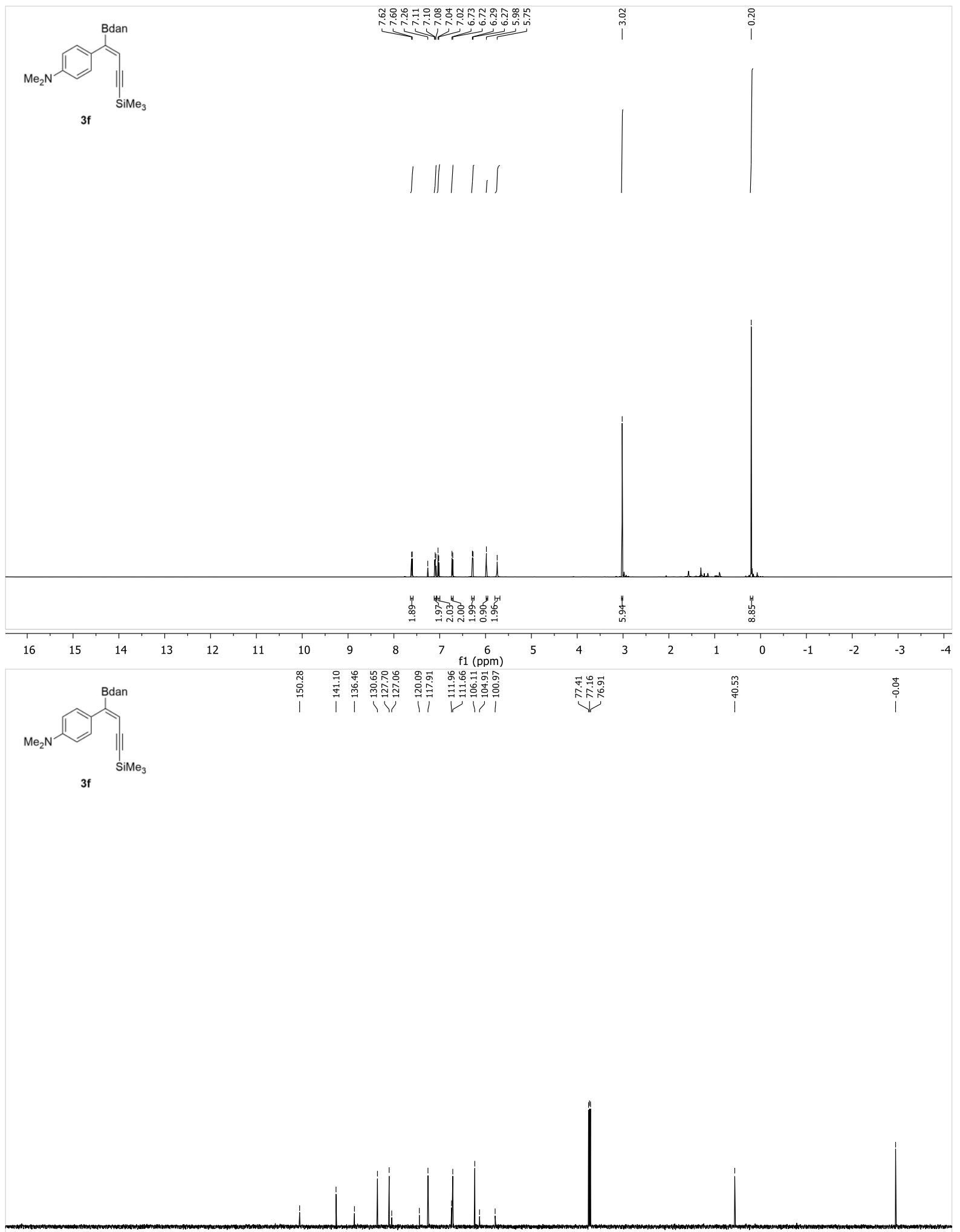

$\begin{array}{lllllllllllllllllllllllllll}220 & 210 & 200 & 190 & 180 & 170 & 160 & 150 & 140 & 130 & 120 & 110 & 100 & 90 & 80 & 70 & 60 & 50 & 40 & 30 & 20 & 10 & 0 & -10\end{array}$ 


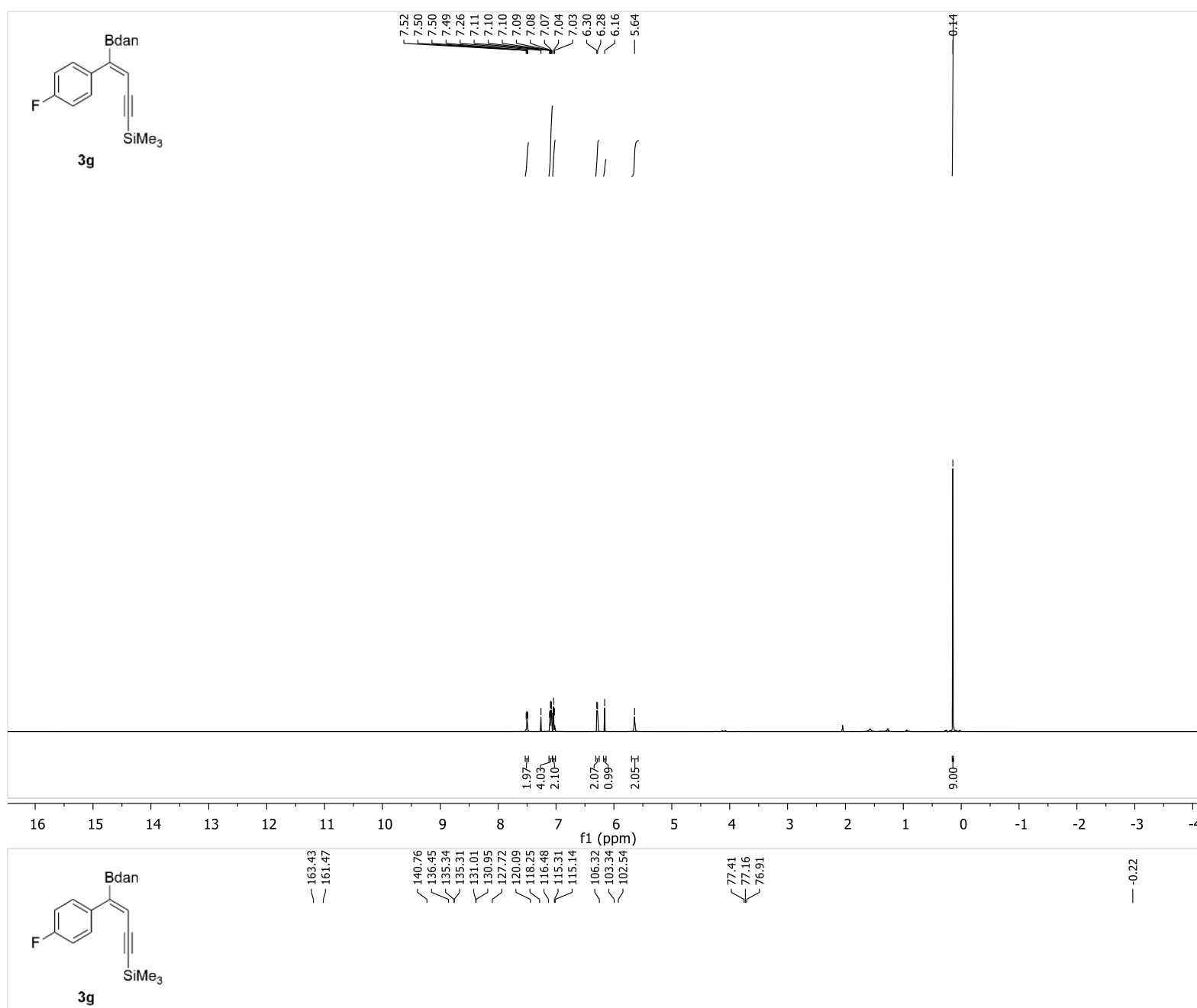

$3 g$

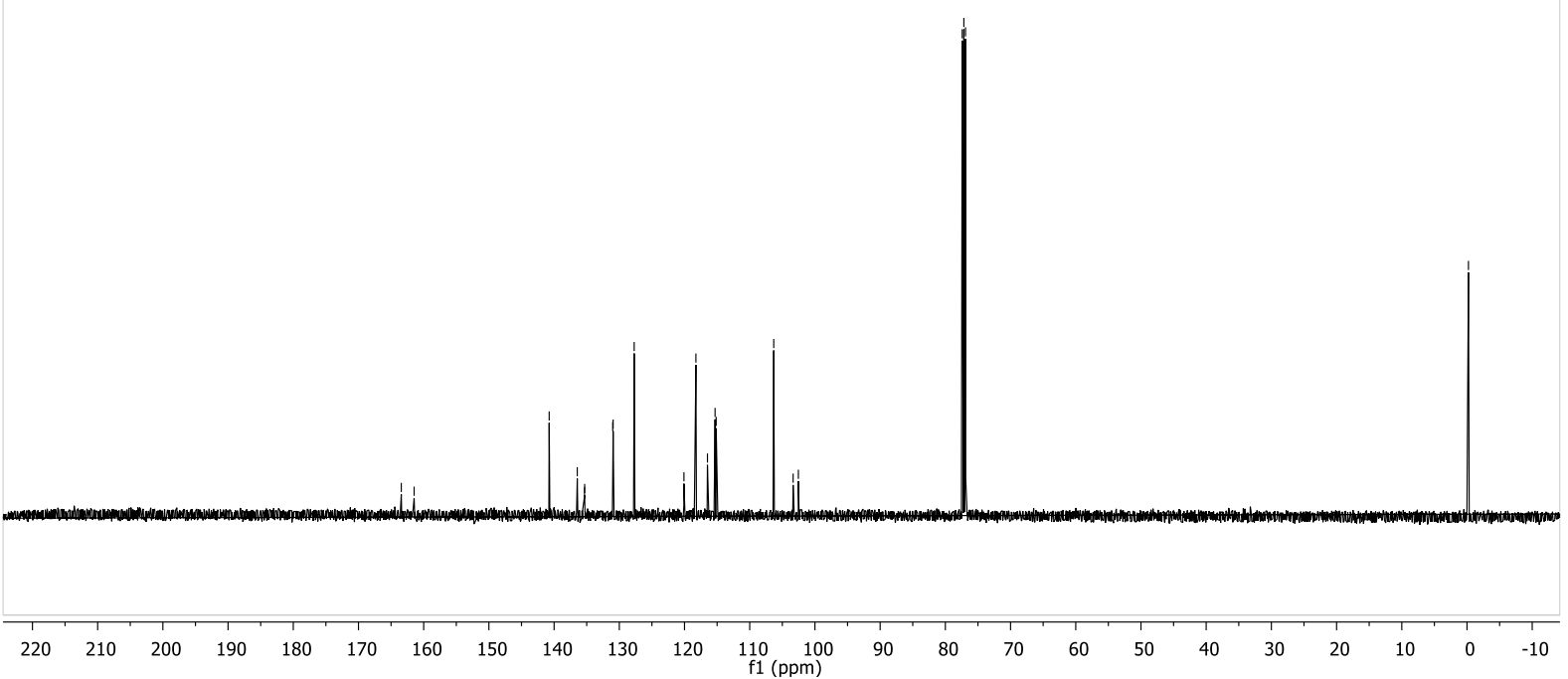




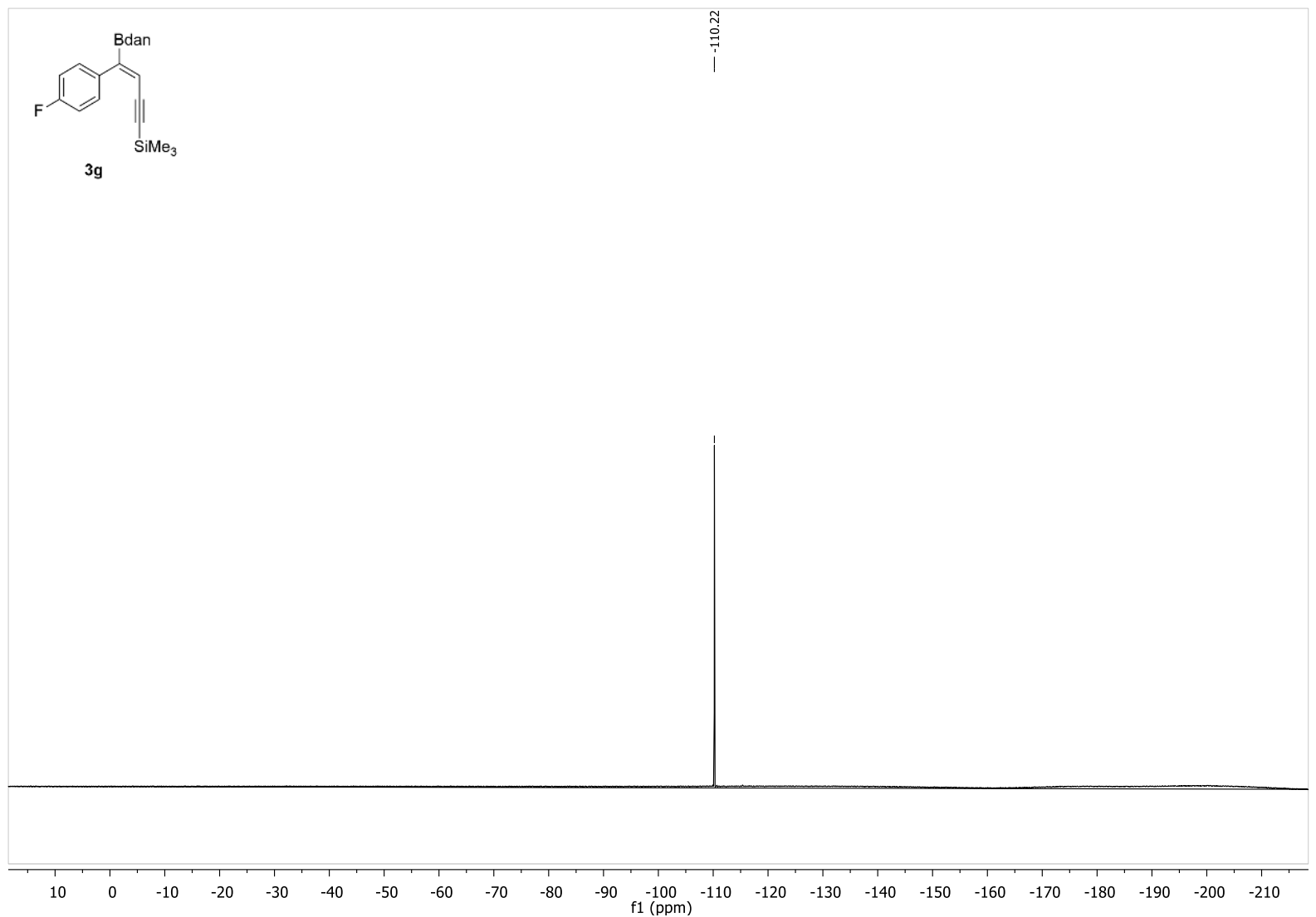




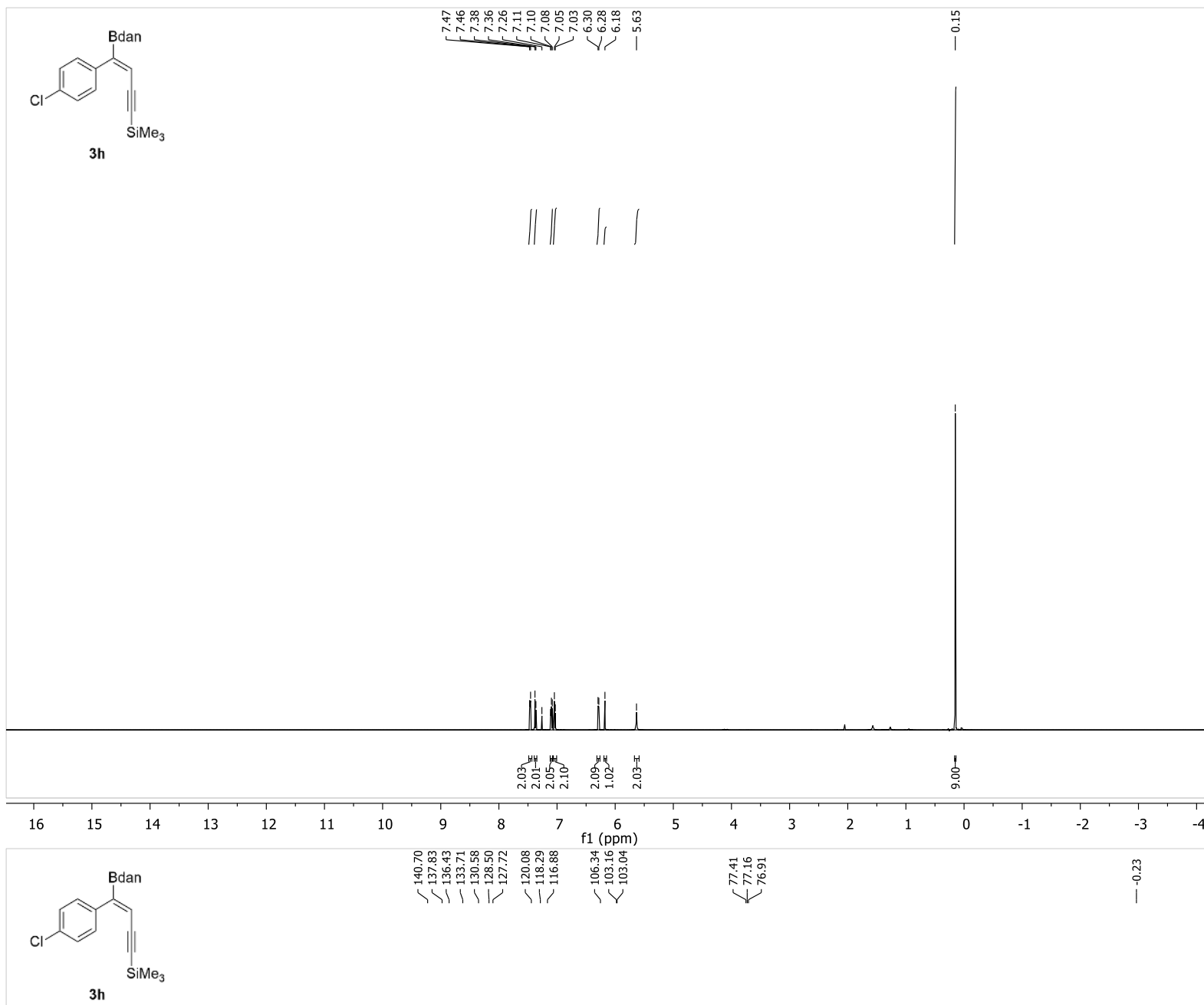

$3 \mathrm{~h}$

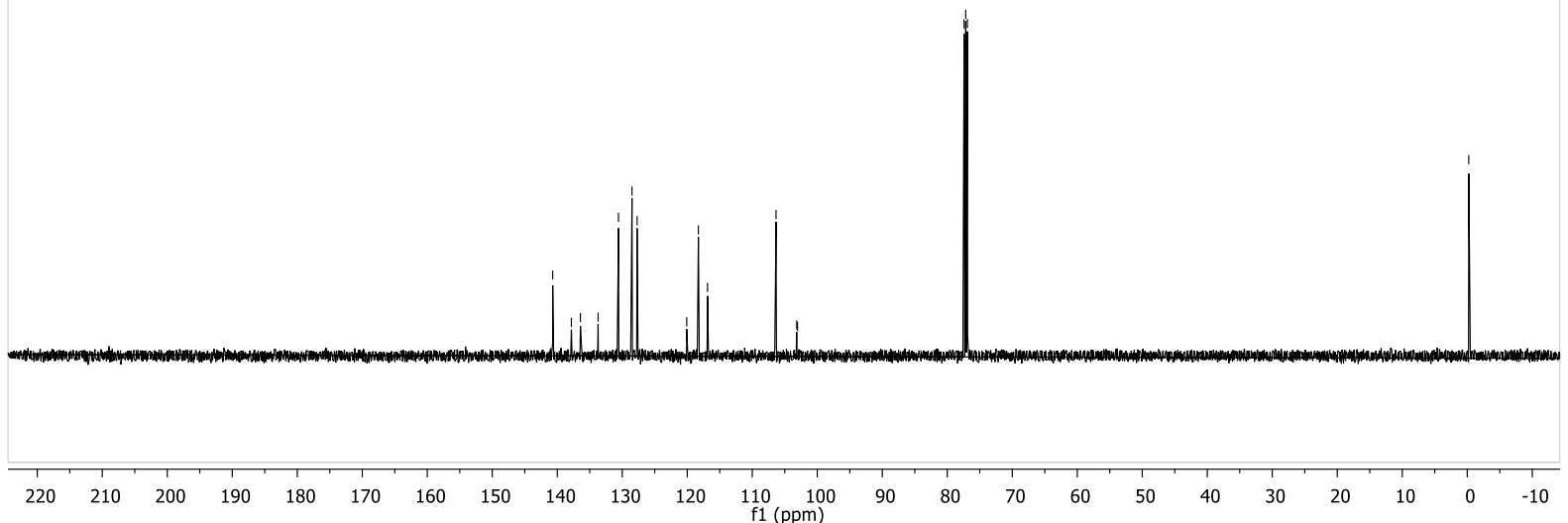



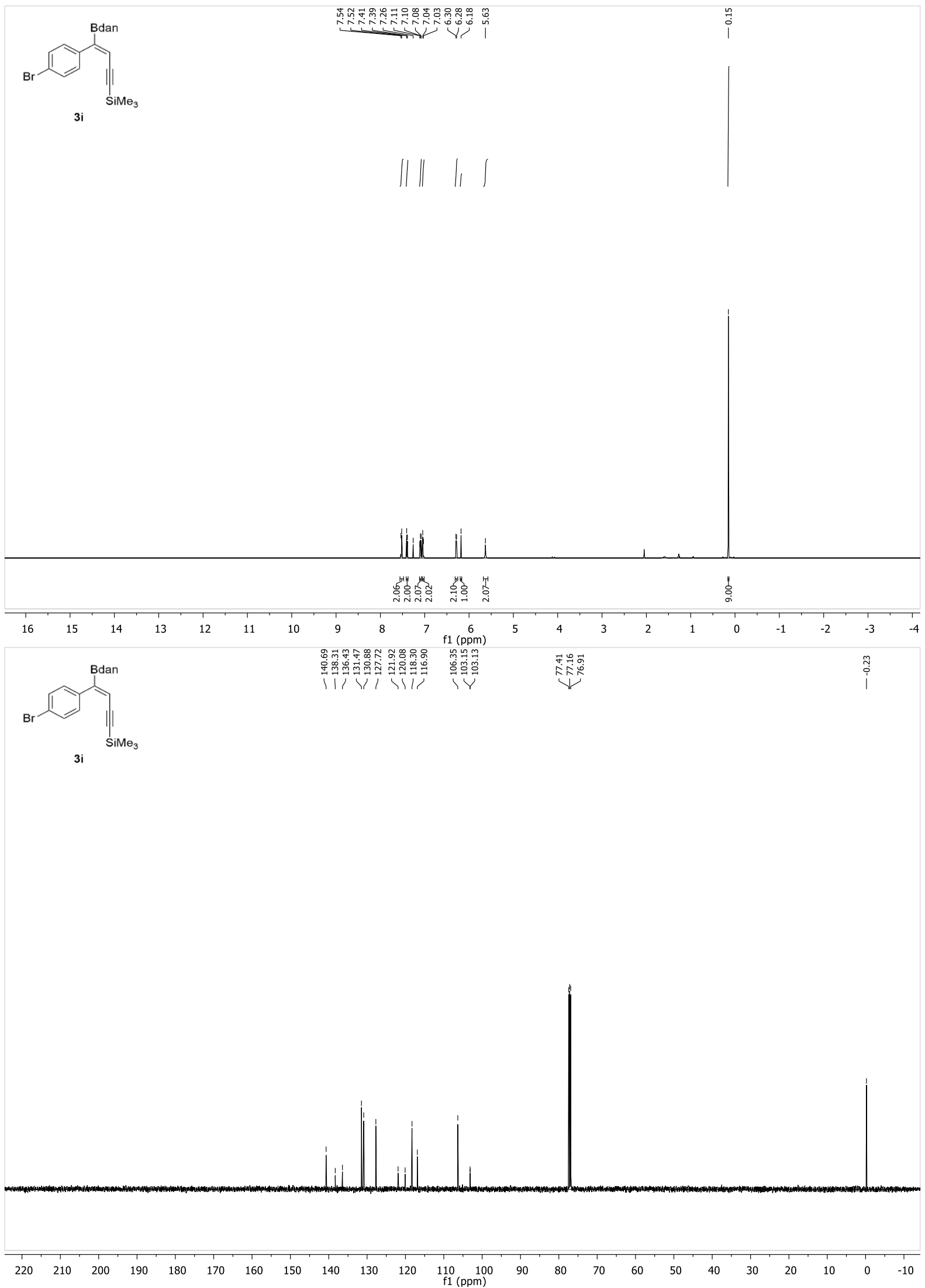


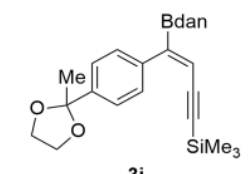

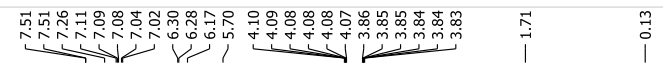

3j
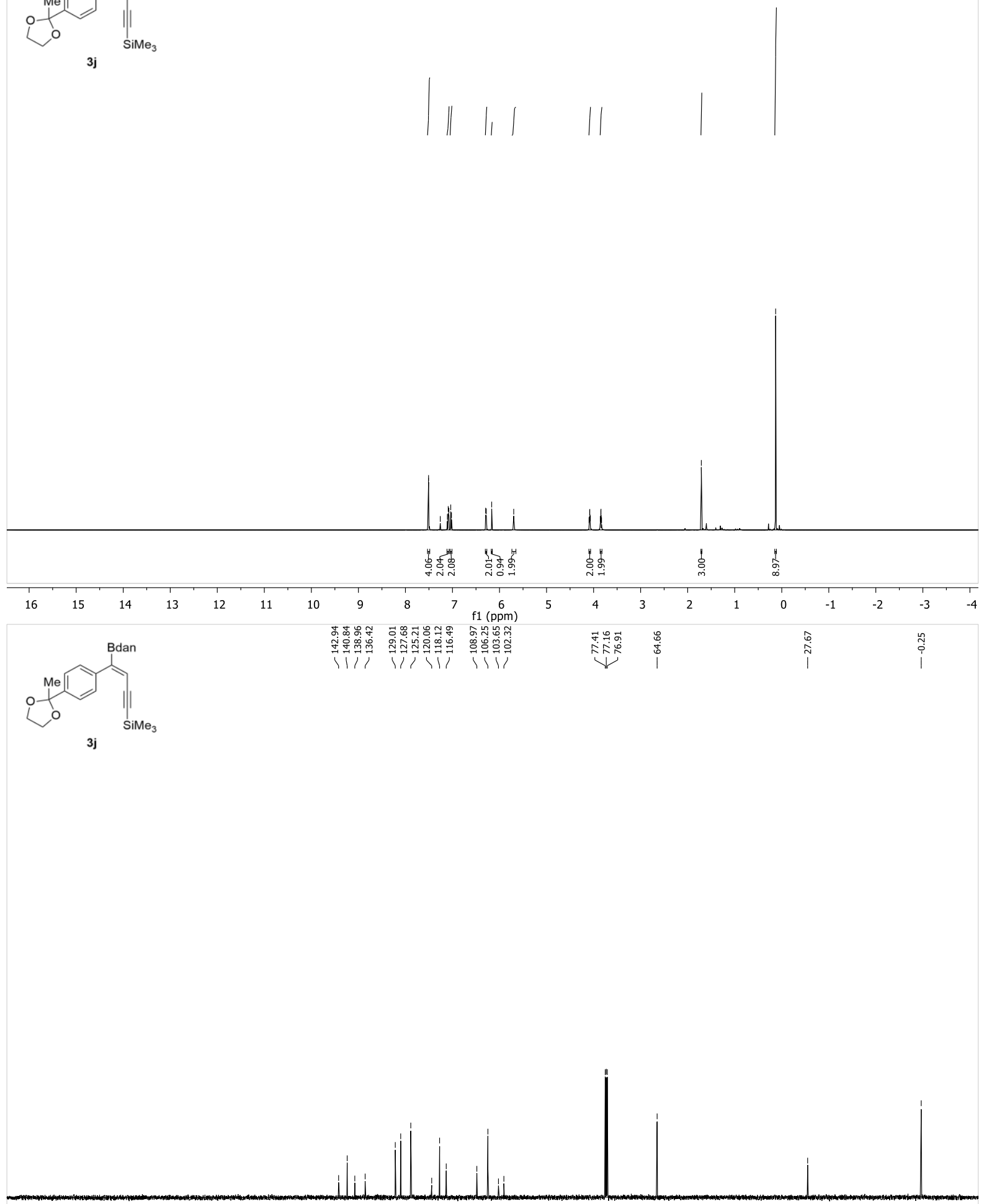

$\begin{array}{llllllllllllllllllllllllll}220 & 210 & 200 & 190 & 180 & 170 & 160 & 150 & 140 & 130 & 120 & 110 & 100 & 90 & 80 & 70 & 60 & 50 & 40 & 30 & 20 & 10 & 0 & -10\end{array}$ 

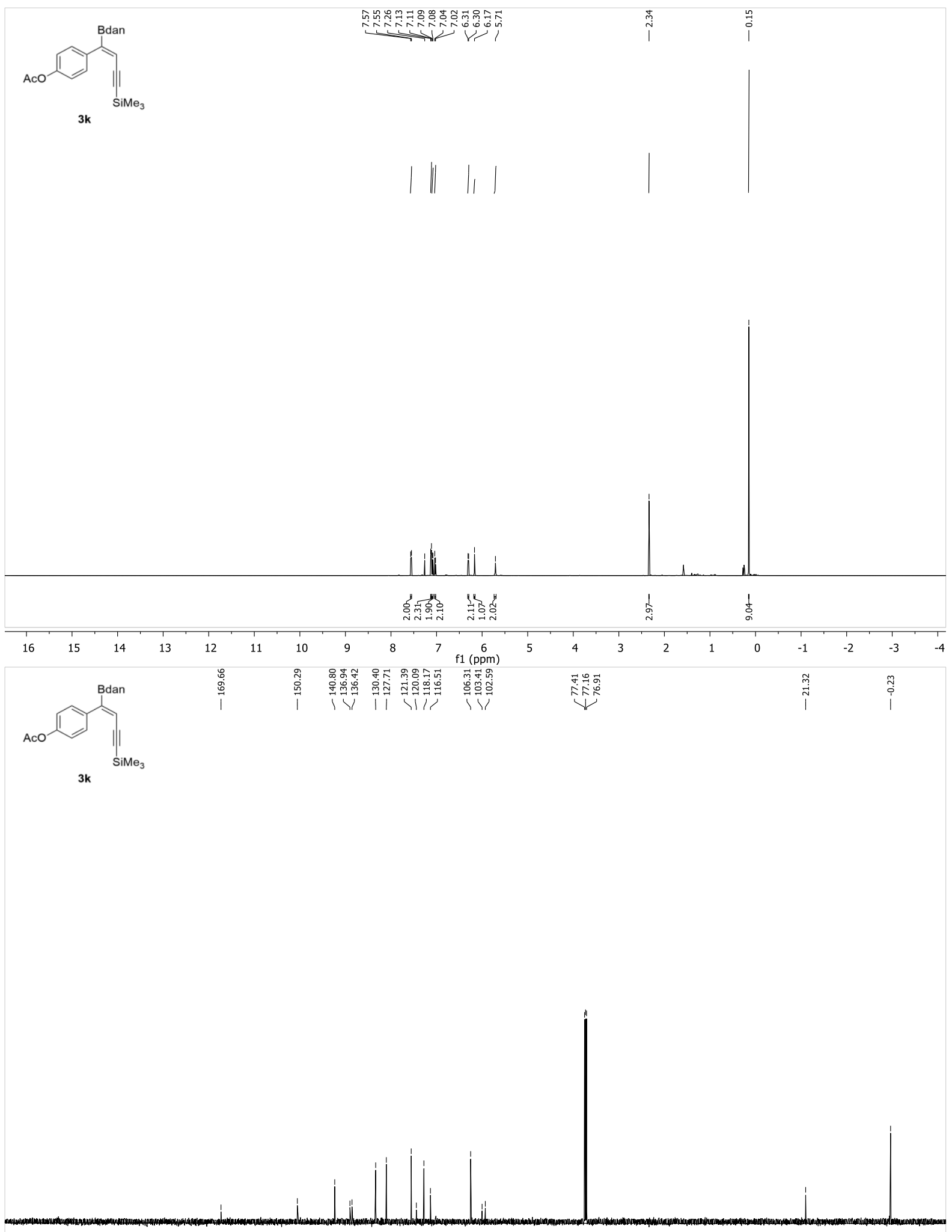

$\begin{array}{lllllllllllll}220 & 210 & 200 & 190 & 180 & 170 & 160 & 150 & 140 & 130 & 120 & 110 & 100\end{array}$ 


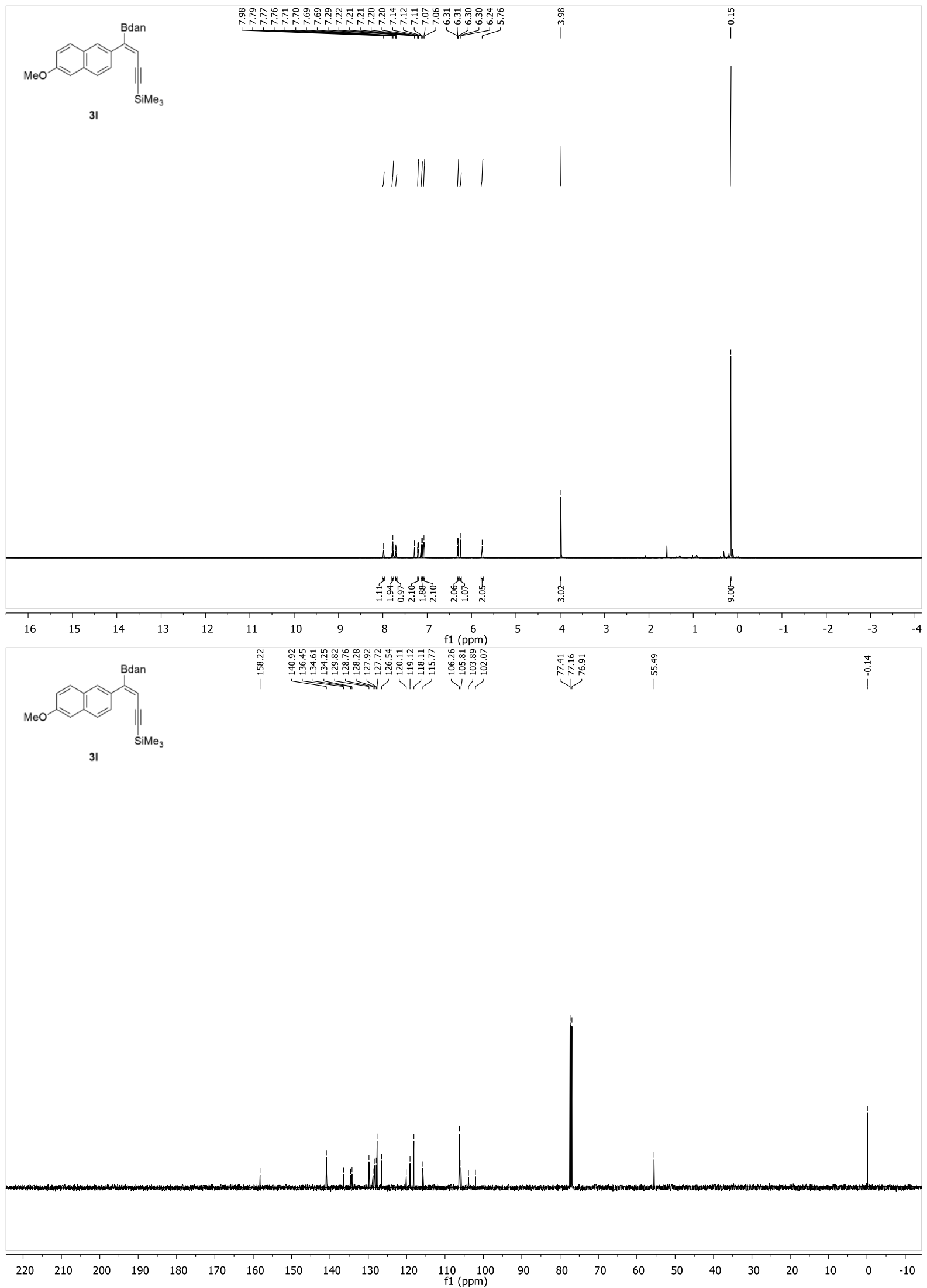




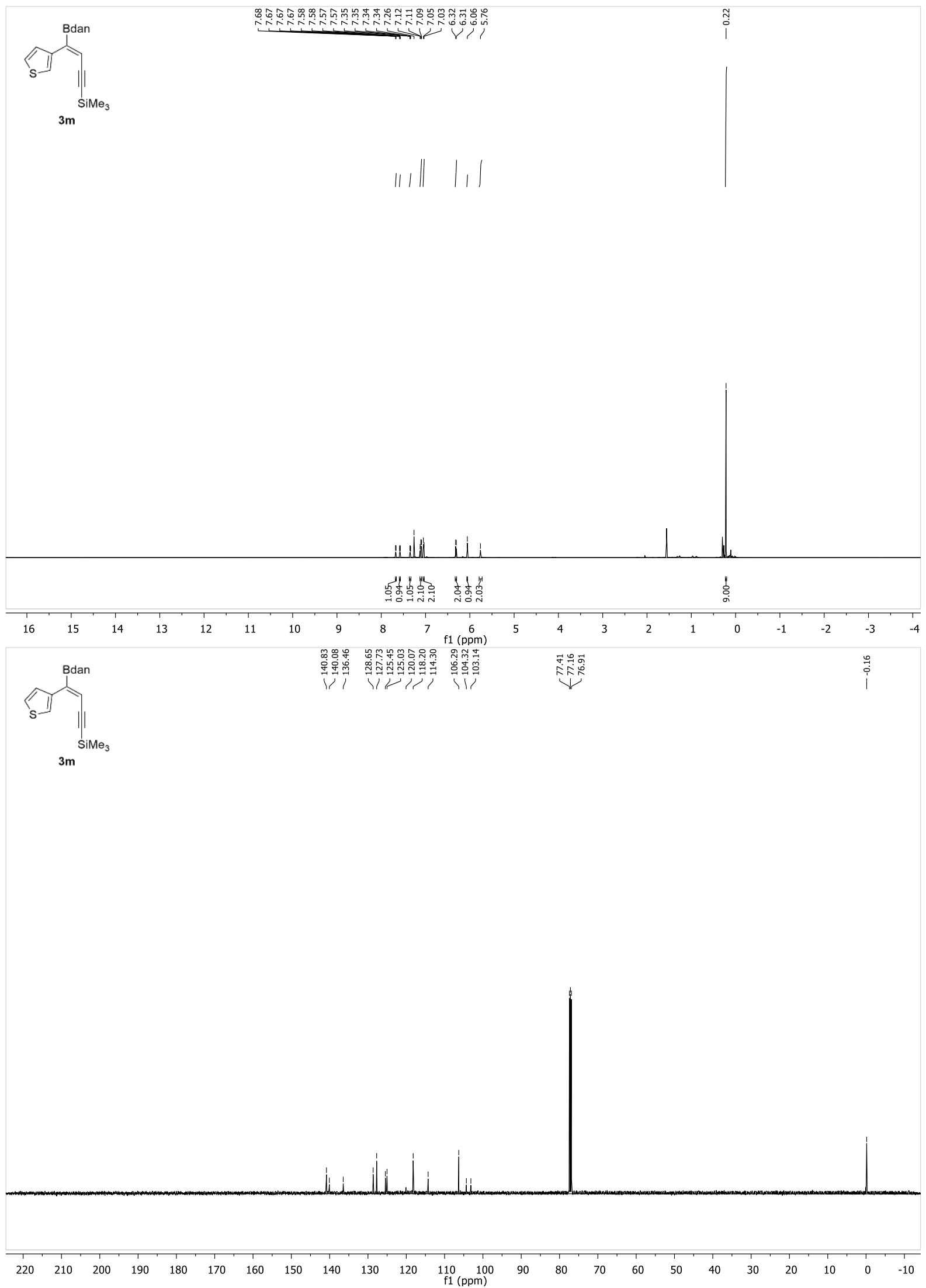



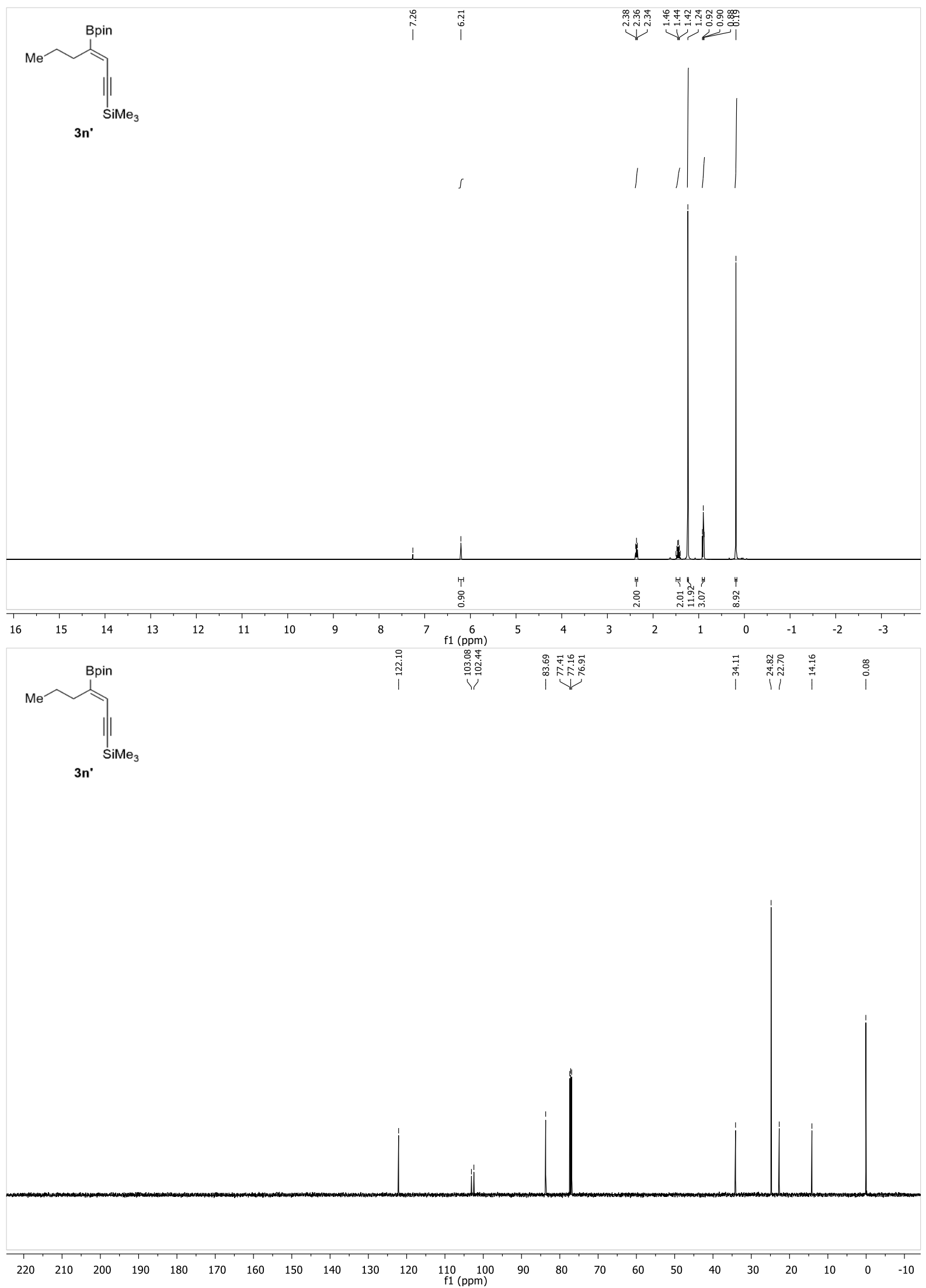

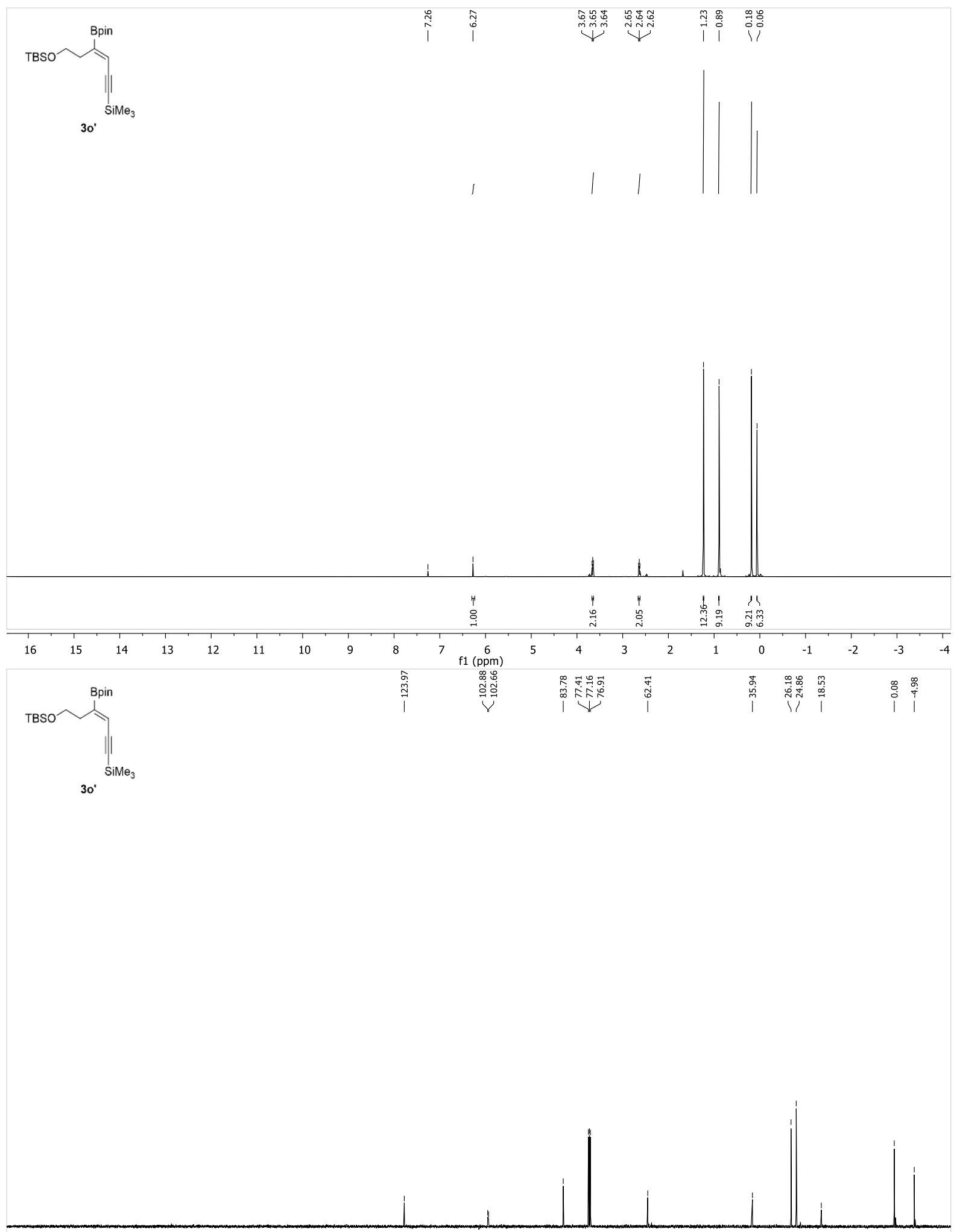

$\begin{array}{llllllllllllllllllllllllllll}220 & 210 & 200 & 190 & 180 & 170 & 160 & 150 & 140 & 130 & 120 & 110 & 100 & 90 & 80 & 70 & 60 & 50 & 40 & 30 & 20 & 10 & 0 & -10\end{array}$ 

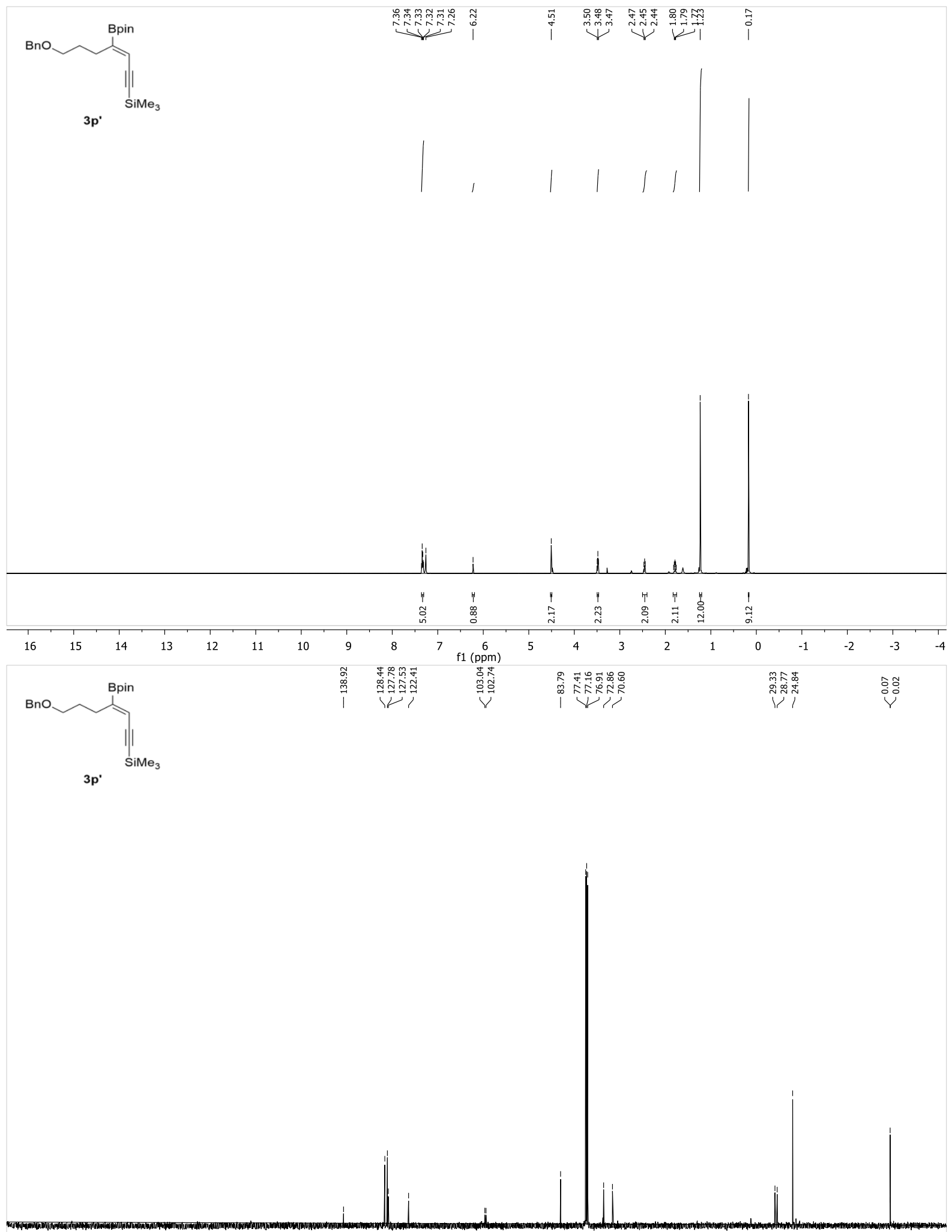

$\begin{array}{llllllllllllllllllllllllllll}220 & 210 & 200 & 190 & 180 & 170 & 160 & 150 & 140 & 130 & 120 & \begin{array}{c}110 \\ \mathrm{f} 1(\mathrm{ppm})\end{array} & 90 & 80 & 70 & 60 & 50 & 40 & 30 & 20 & 10 & 0 & -10\end{array}$ 


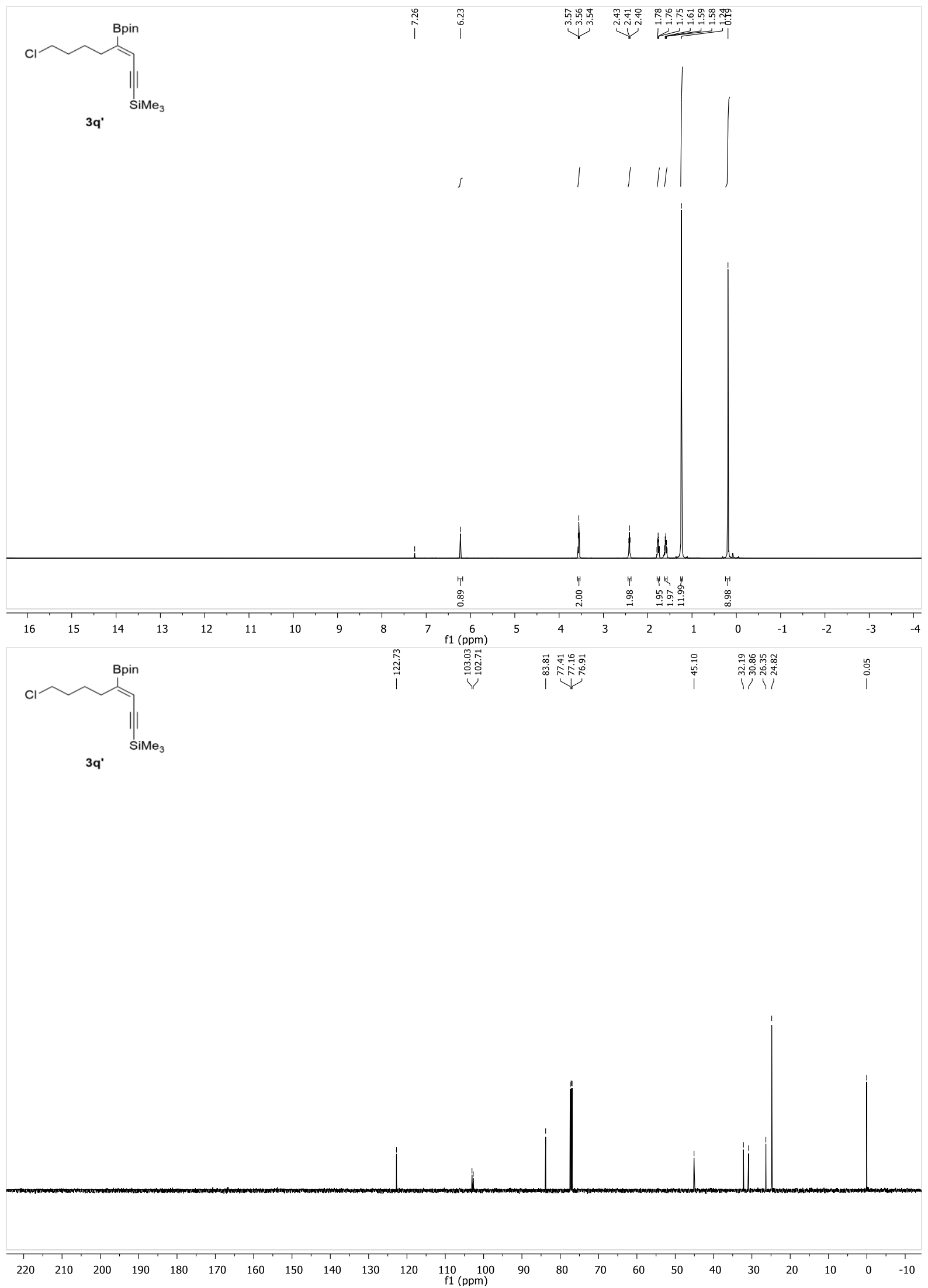



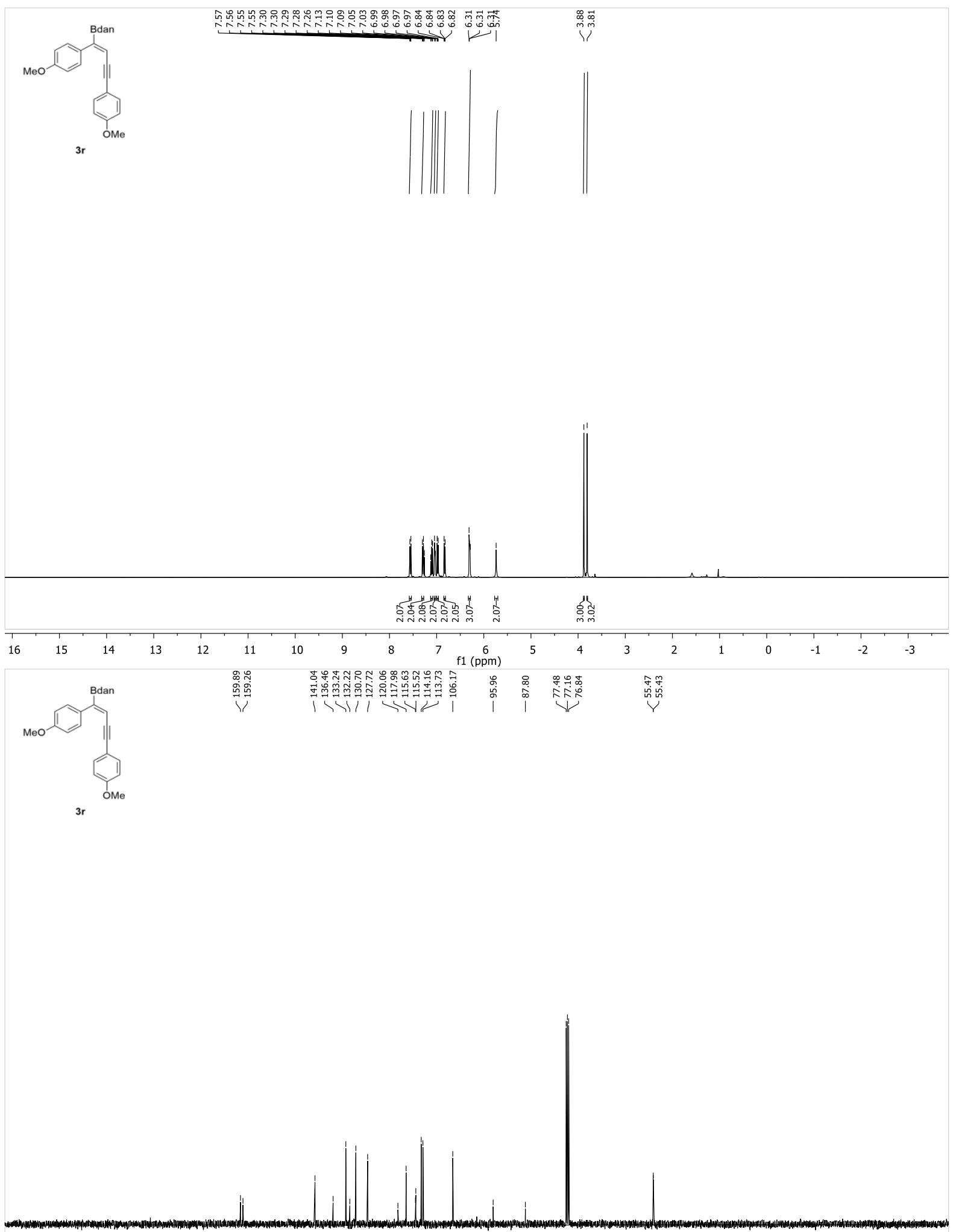

$\begin{array}{lllllllllllllllllllllll}210 & 200 & 190 & 180 & 170 & 160 & 150 & 140 & 130 & 120 & 110 & 100 & 90 & 80 & 70 & 60 & 50 & 40 & 30 & 20 & 10 & 0 & -10\end{array}$ 


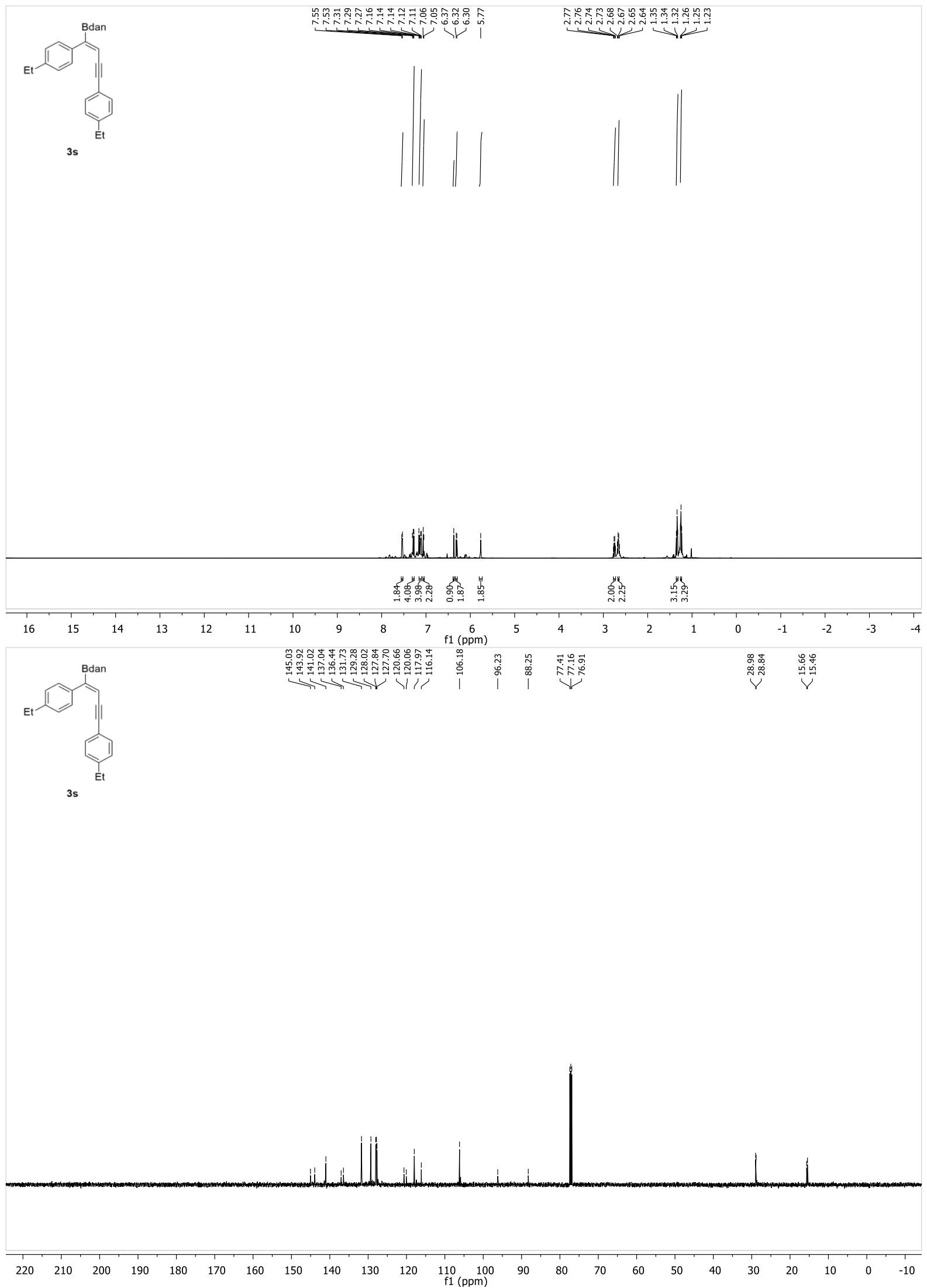



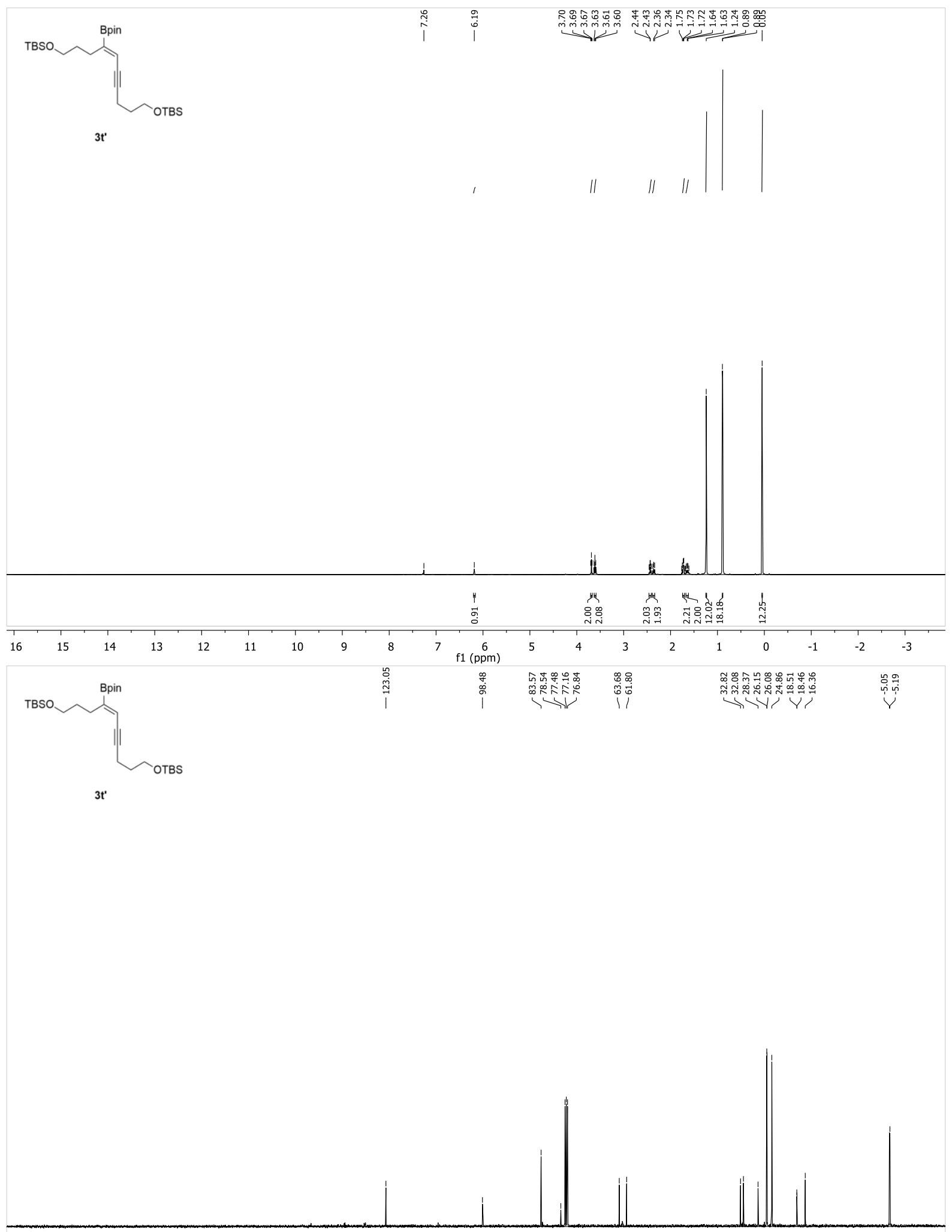

$\begin{array}{lllllllllllllllllllllll}210 & 200 & 190 & 180 & 170 & 160 & 150 & 140 & 130 & 120 & 110 & \underset{\mathrm{f} 1(\mathrm{ppm})}{100} & 90 & 80 & 70 & 60 & 50 & 40 & 30 & 20 & 10 & 0 & -10\end{array}$ 


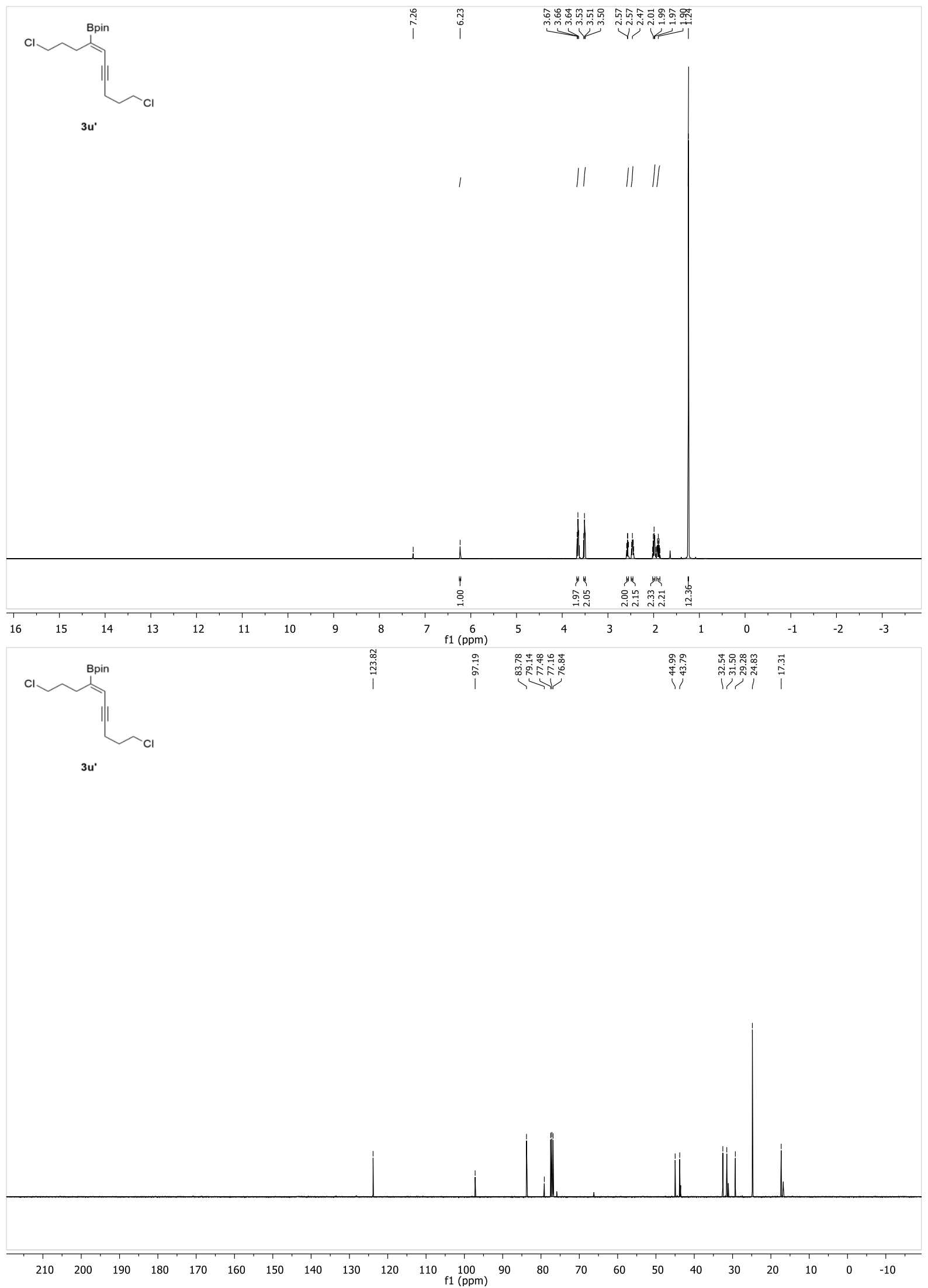




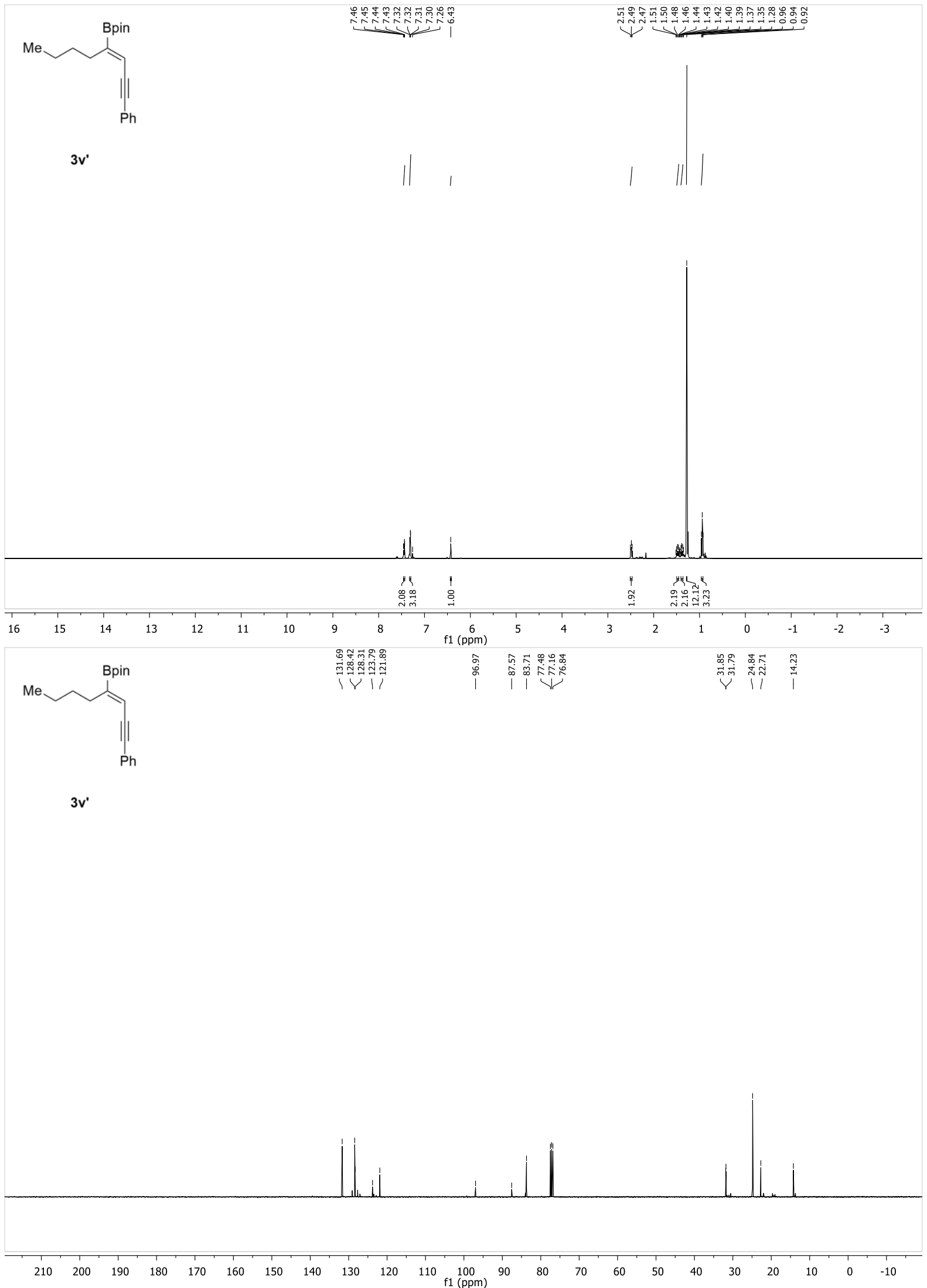




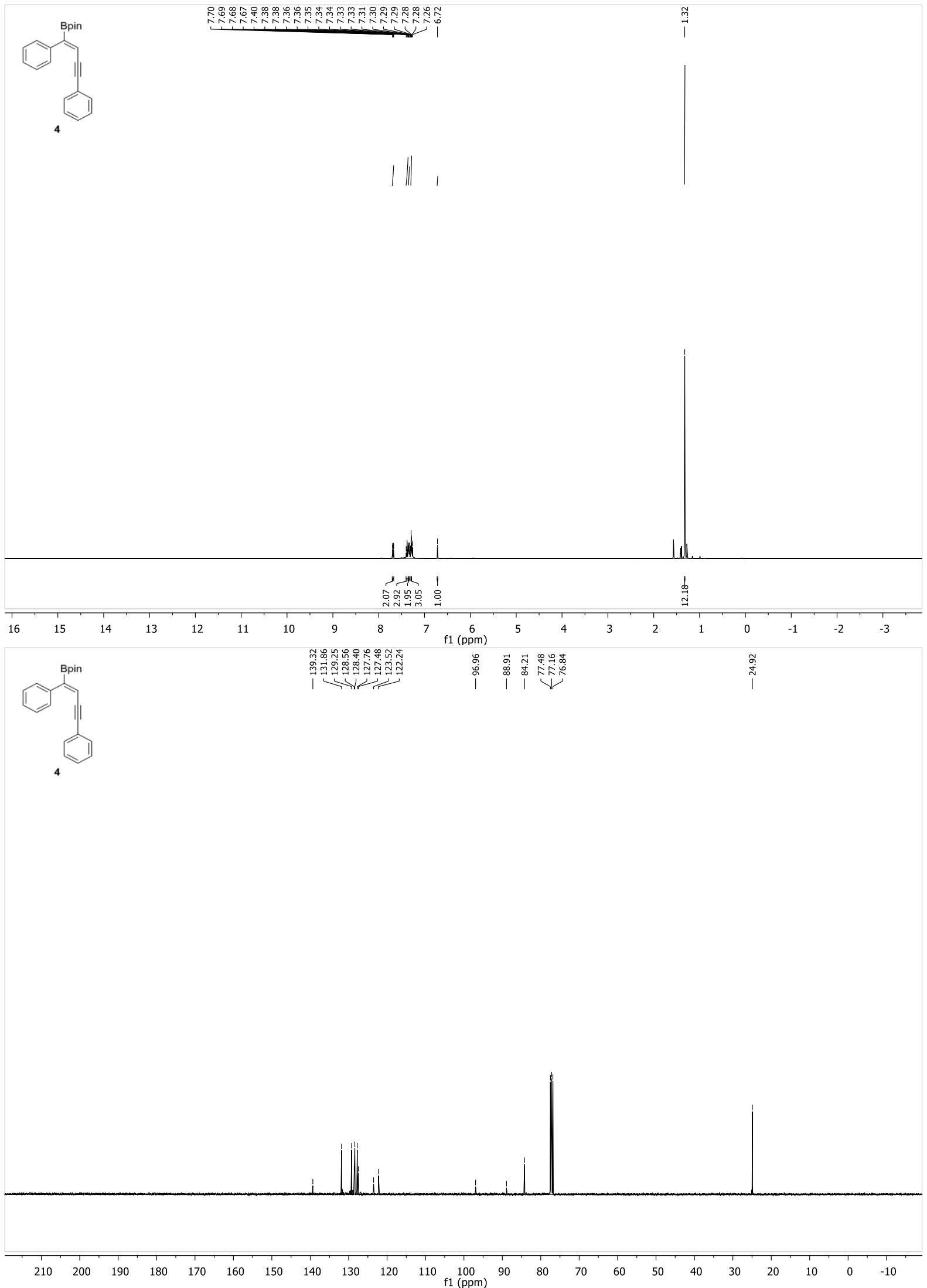




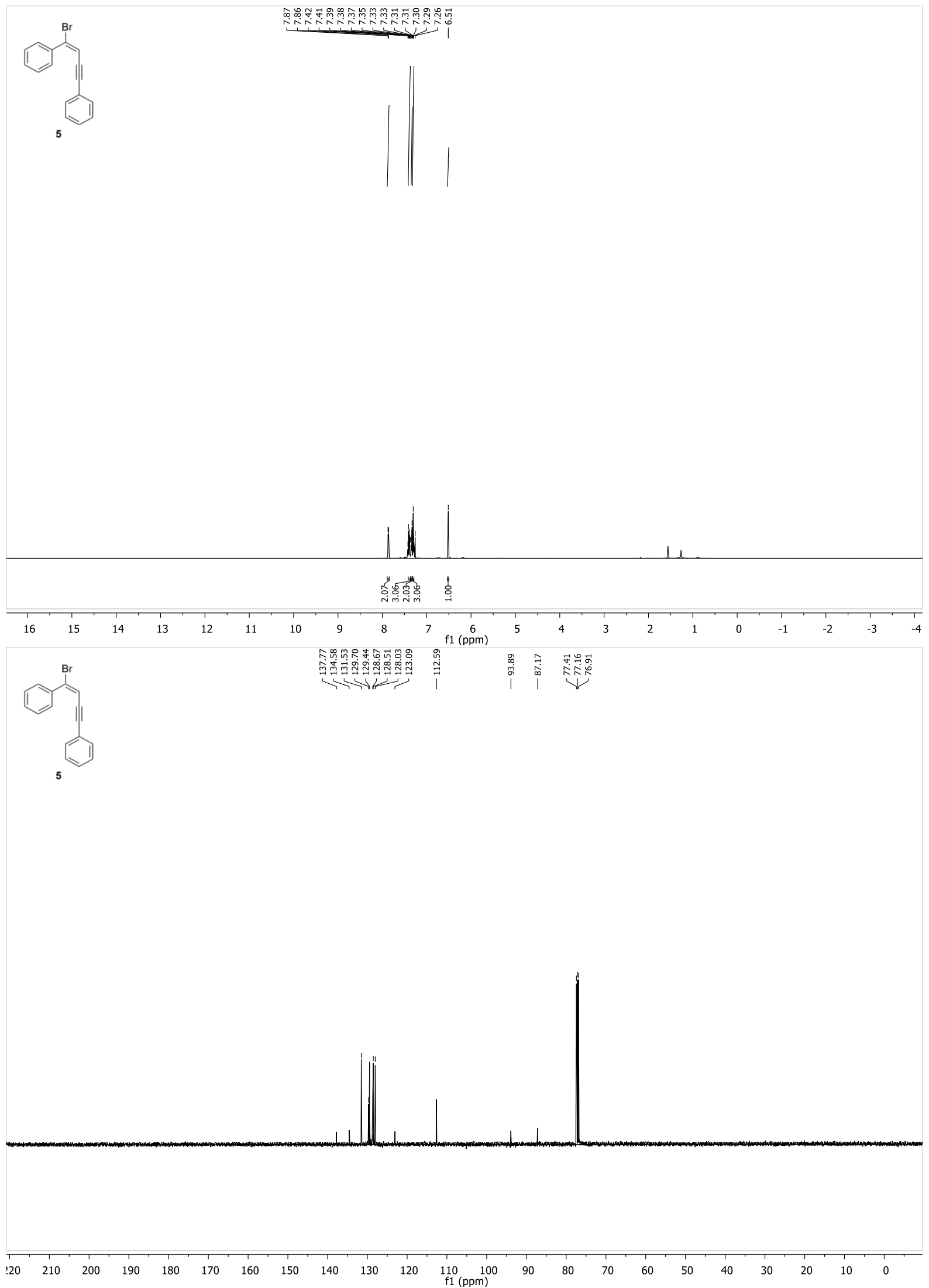




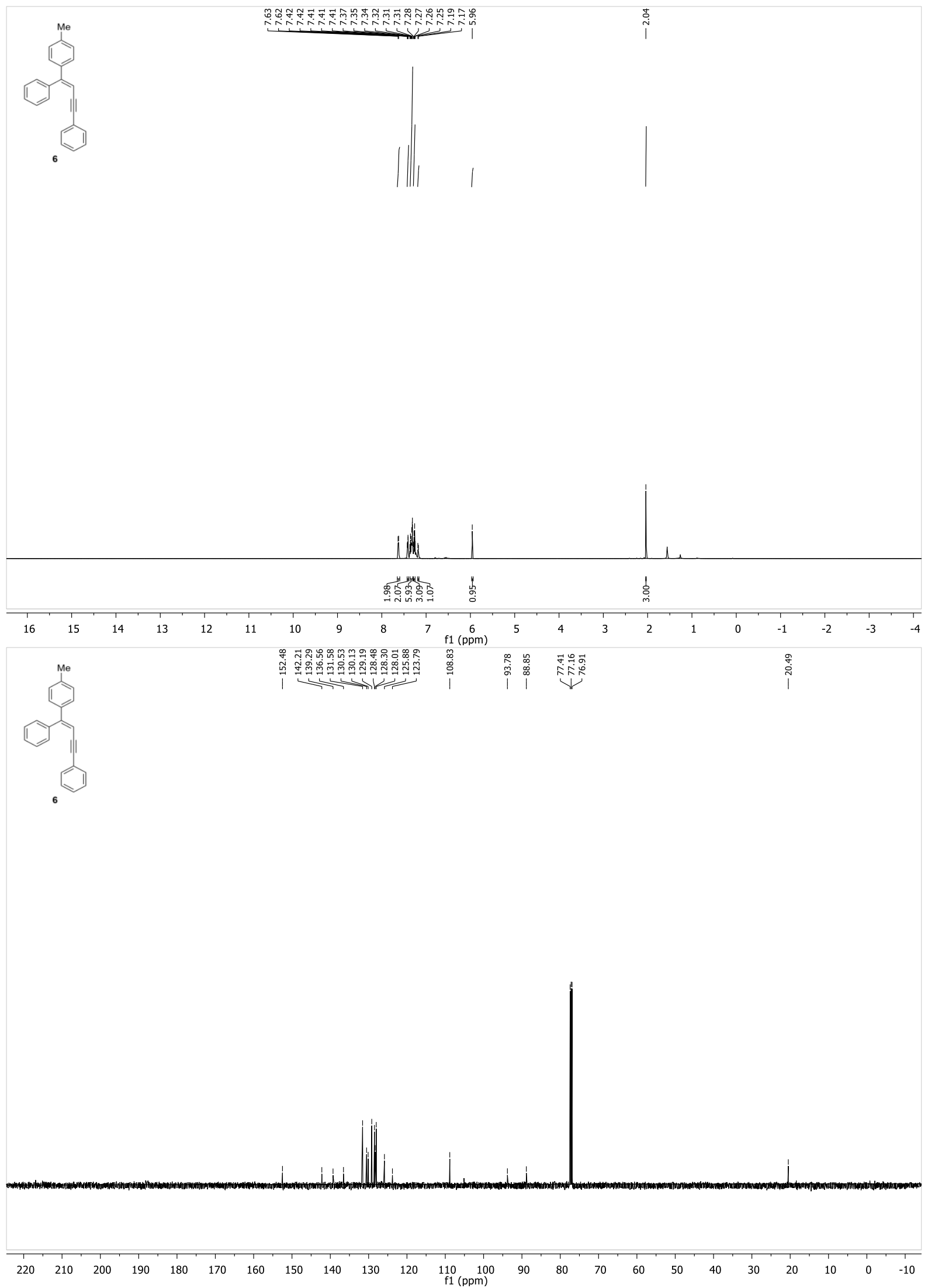




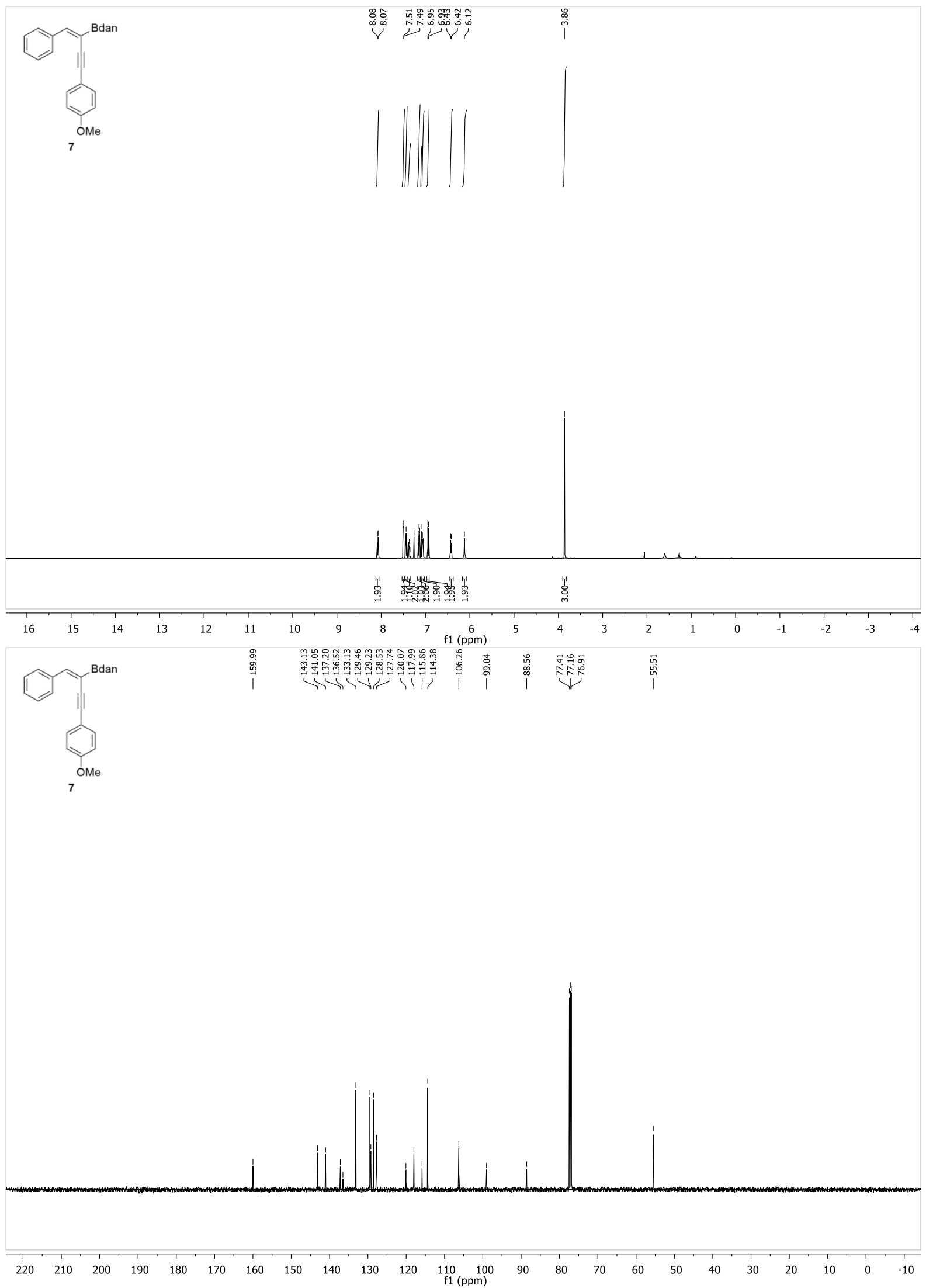




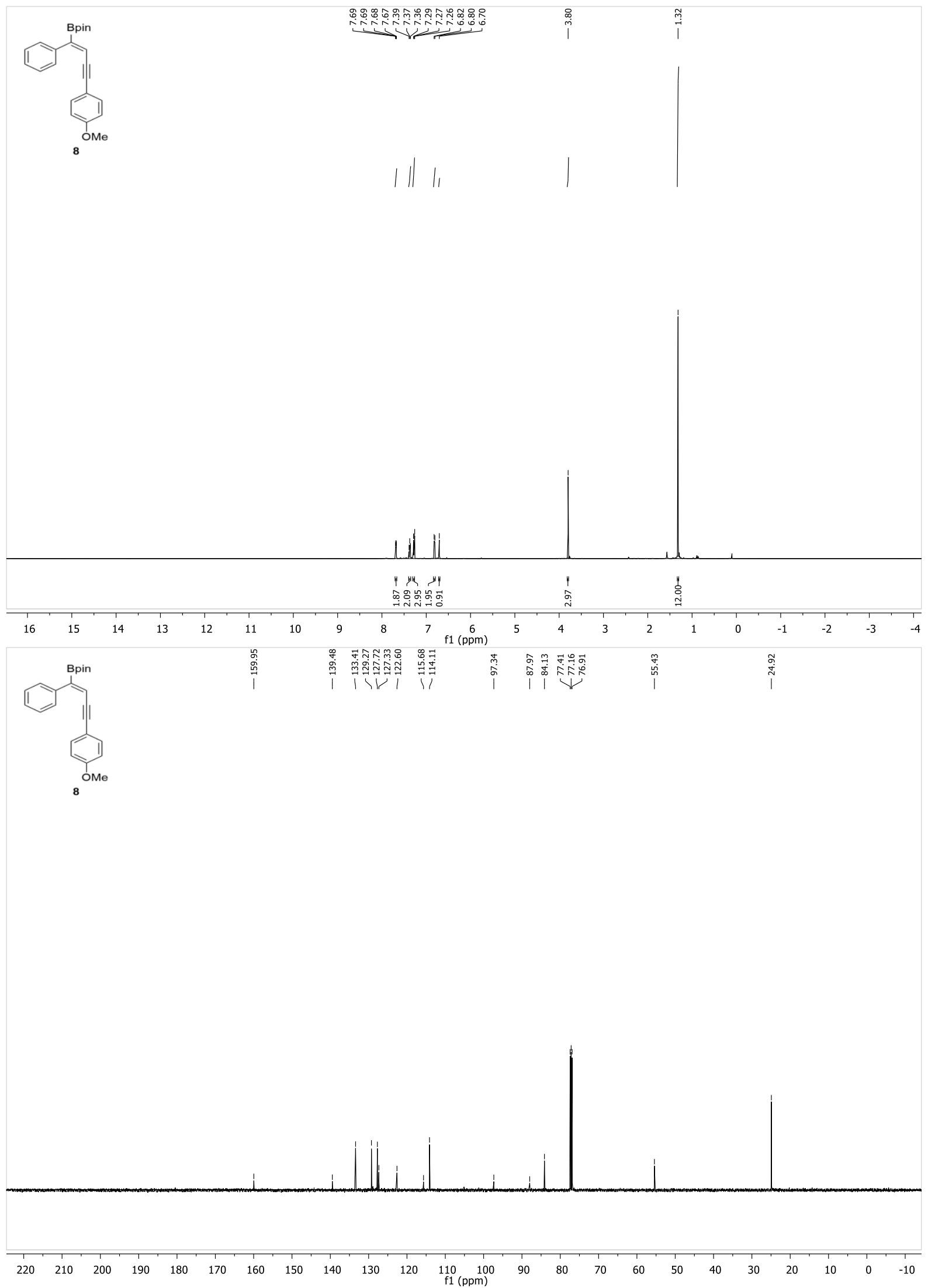

